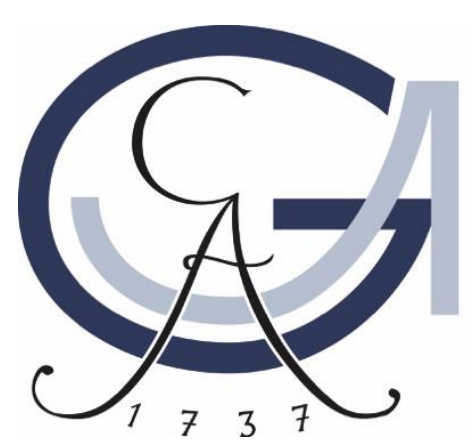

\title{
Mechanisms of regulation of mitochondria-endoplasmic reticulum contact sites
}

\author{
Dissertation \\ for the award of the degree \\ "Doctor of Philosophy" \\ of the Georg-August-Universität Göttingen \\ within the doctoral program Molecular Biology of Cells \\ of the Georg-August University School of Science (GAUSS)
}

submitted by

Renata Lopes Familiar Couto

from Santa Maria da Feira, Portugal

Göttingen, 2019 


\section{Members of the Thesis Committee}

Dr. Nuno Raimundo ( $1^{\text {st }}$ reviewer)

Department of Cellular Biochemistry

University Medical Center Göttingen

Prof. Dr. Blanche Schwappach-Pignataro ( ${ }^{\text {nd }}$ reviewer)

Department of Molecular Biology

University Medical Center Göttingen

\section{Dr. Alexander Stein}

Membrane Protein Biochemistry Group

Max Planck Institute for Biophysical Chemistry

Further members of the Examination Board

Dr. Ira Milosevic

Synaptic Vesicle Dynamics Group

European Neuroscience Institute Göttingen

Prof. Dr. Silvio Rizzolli

Department of Neuro- and Sensory Physiology

University Medical Center Göttingen

Prof. Dr. Doerthe Katschinski

Institute of Cardiovascular Physiology

University Medical Center Göttingen

Date of oral examination: $28^{\text {th }}$ October 2019 


\section{Declaration}

I hereby declare that the dissertation, entitled 'Mechanisms of regulation of mitochondriaendoplasmic reticulum contact sites', was written independently and with no other aids or sources than quoted.

Renata Couto

Göttingen, August 2019 
"O Homem é do tamanho do seu sonho." Man is the size of his dream.

Fernando Pessoa

"Science is not only a disciple of reason but also one of romance and passion."

Stephen Hawking

"Nothing in life is to be feared, it is only to be understood. Now is the time to understand more, so that we may fear less." Marie Curie 


\section{Acknowledgments}

First of all, I would like to thank my supervisor, Dr. Nuno Raimundo, for giving me this great opportunity with a challenging and exciting project. I thank him for all the support and motivation during all this time.

To the members of my thesis committee, Prof. Dr. Blanche Schwappach-Pignataro and Dr. Alexander Stein, I thank their participation in my Ph.D. thesis, their suggestions and advices, and for the overall support. Also, I would like to thank the further members of my examination board, Dr. Ira Milosevic, Prof. Dr. Silvio Rizzolli and Prof. Dr. Doerthe Katschinski, for their time and support. I also would like to thank all the collaborators who contributed for this project.

To all present and past members from Raimundo and Milosevic labs, I would like to thank for all the support along these years, either science or social related and for the great environment in the lab. I am very grateful to King for all experimental support, meaningful discussions, as well as for the proofreading of this thesis. I also would like to give a special thank you to Sindhu for the great advices and the help in experiments along my work.

This long way allowed me to make new friends, to strengthen some others or even lose some, but one thing I am sure, every single person that cross my way during this time contributed for the dream become true, even with the smallest thing! And to them I would like to thank for all the support and motivation, for taking care of me when I needed the most and for all the great moments we had. For you Maria, Cátia, Sílvia, Floriane and Anita thank you for your friendship and for your lovely presence in my life.

Por último e mais importante, agradeço às pessoas mais importantes da minha vida, os meus pais e o meu tão adorado irmão. É a eles que tenho a agradecer toda a força e apoio incondicional que me deram para concluir mais uma etapa na minha vida. Com eles ao meu lado e a motivação que me dão, acredito que todos os sonhos e desafios são alcançáveis. Obrigada meus pais por tão bem me ensinarem o quanto devemos lutar pelos nossos sonhos e nunca desistir por mais difícil que $o$ caminho seja. A vós que sempre acreditastes que seria possível, dedico este trabalho, tornando-o nosso.

"One, remember to look up at the stars and not down at your feet. Two, never give up work. Work gives you meaning and purpose, and life is empty without it. Three, if you are lucky enough to find love, remember it is there and don't throw it away. "- Stephen Hawking 


\section{Table of contents}

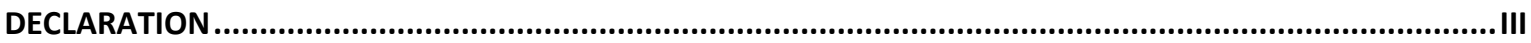

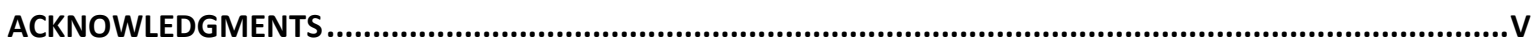

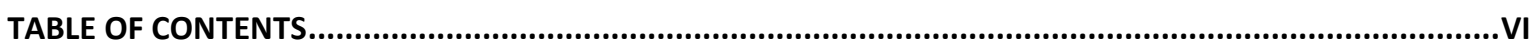

LIST OF FIGURES......................................................................................................................................III

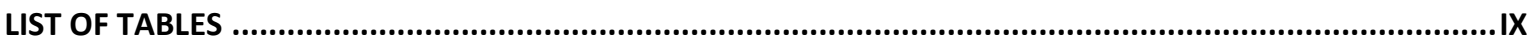

LIST OF ABBREVIATIONS ............................................................................................................................

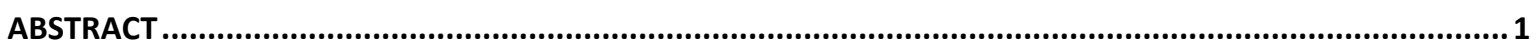

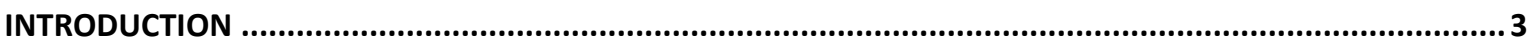

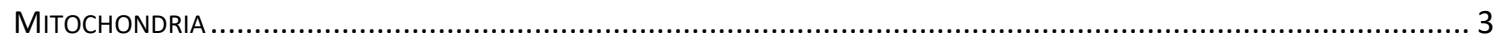

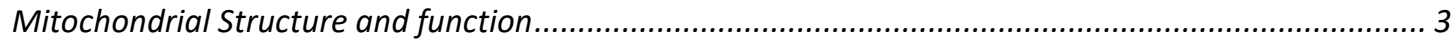

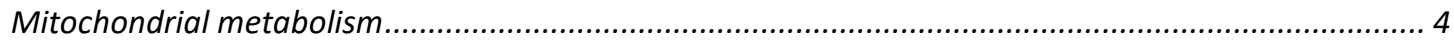

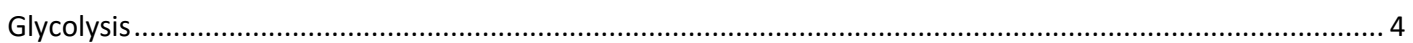

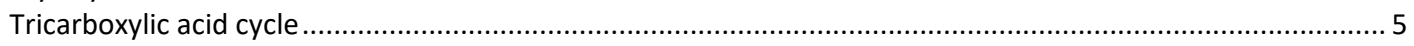

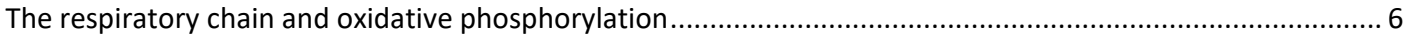

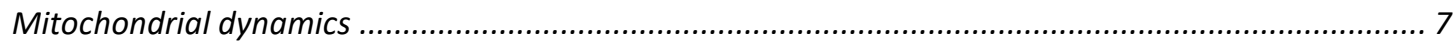

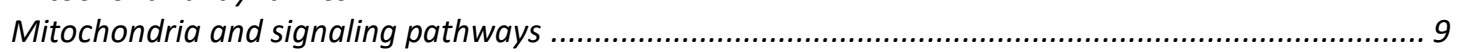

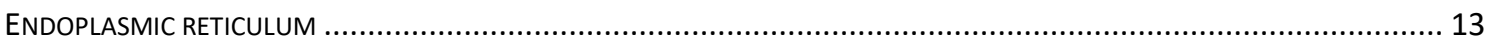

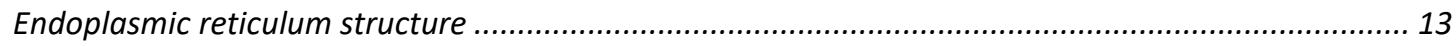

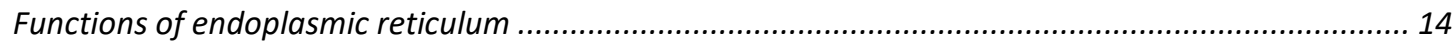

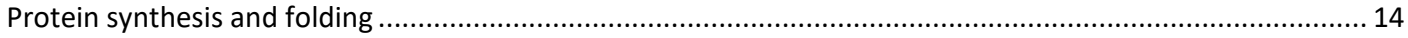

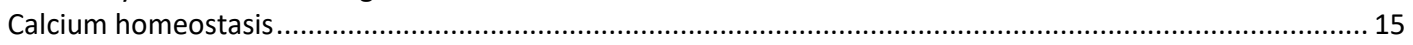

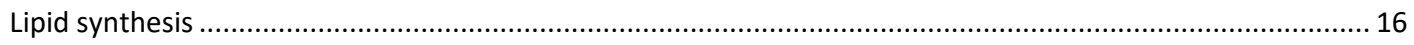

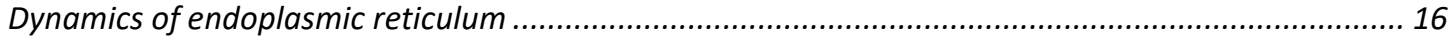

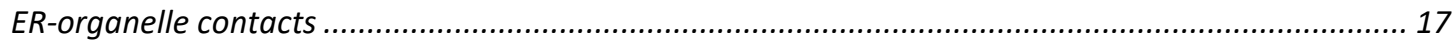

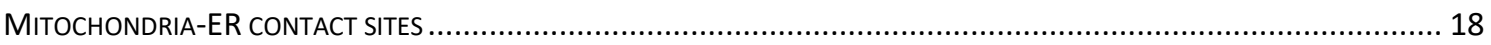

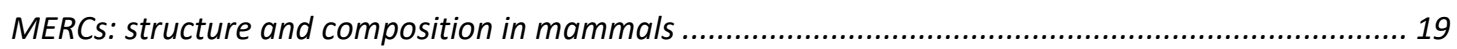

Control of cellular functions by MERCs in mammals..................................................................... 21

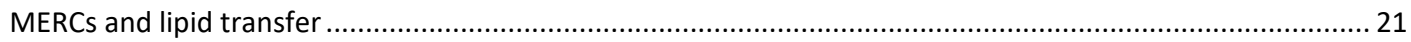

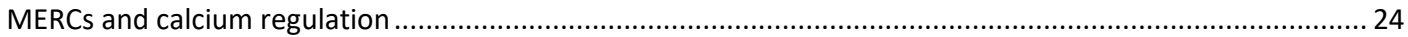

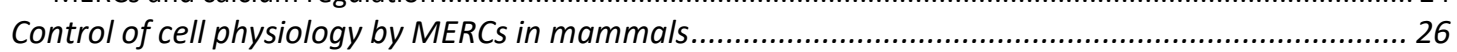

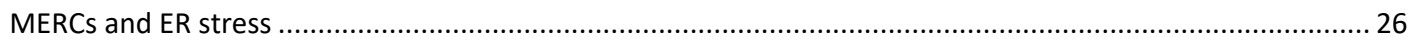

MERCs in autophagy

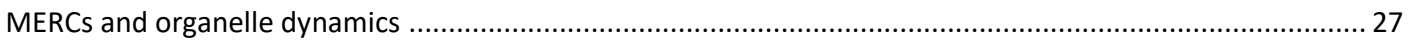

Organelle pathology: MERCs in disease ......................................................................................... 28

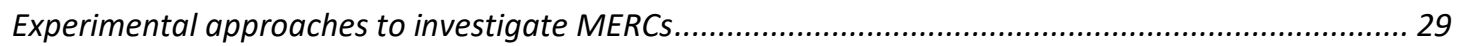

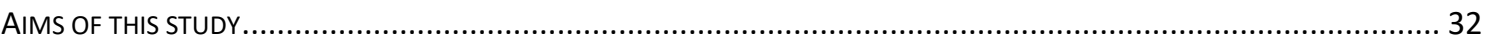

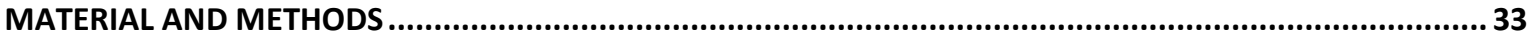

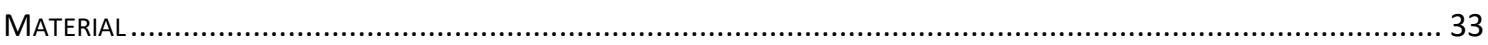

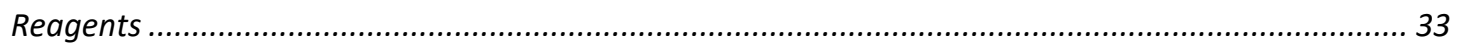

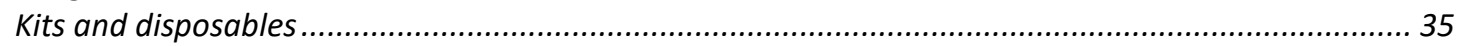

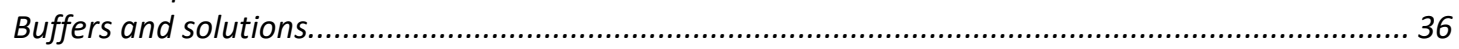

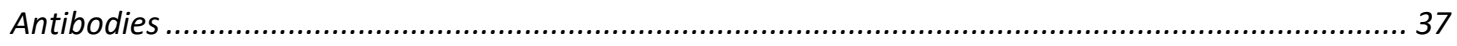

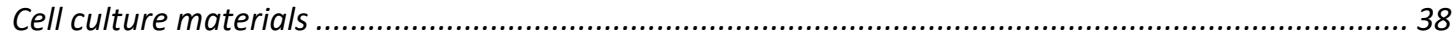

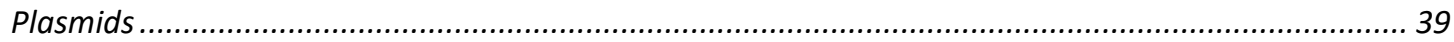

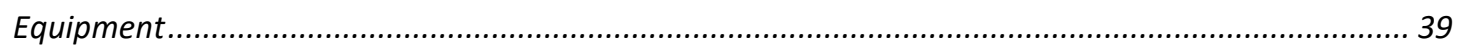

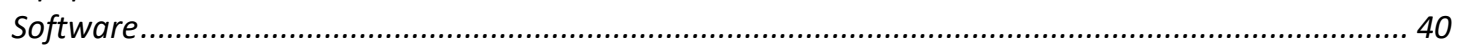

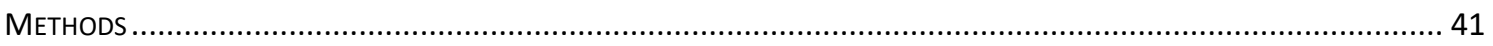

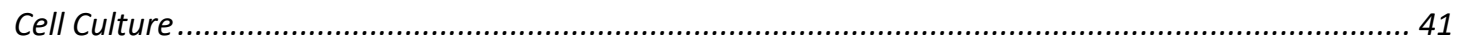

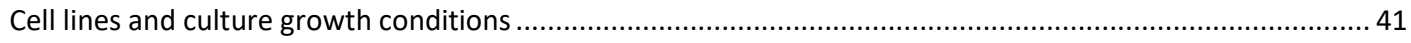

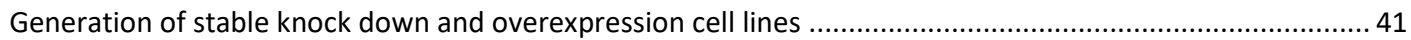

Transfection of HeLa RC-kd and mouse embryonic fibroblasts................................................................. 42 


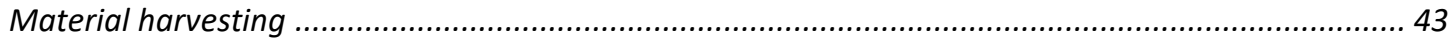

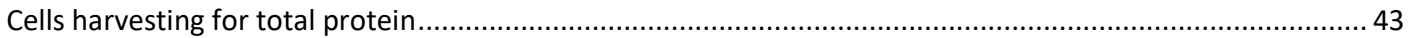

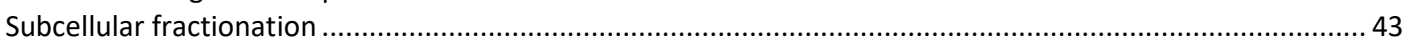

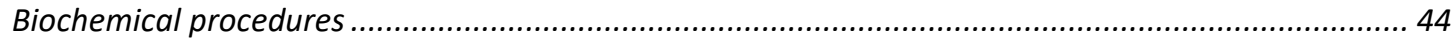

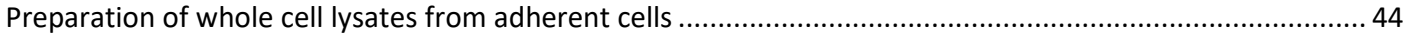

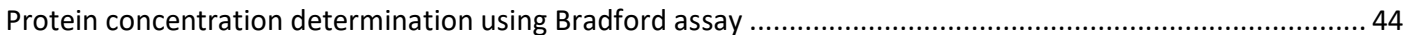

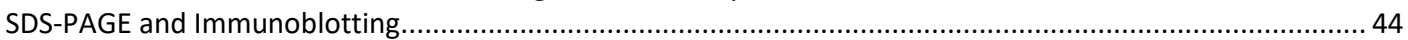

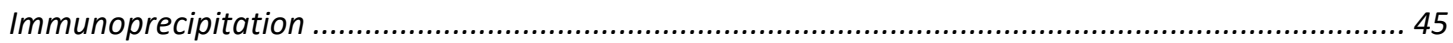

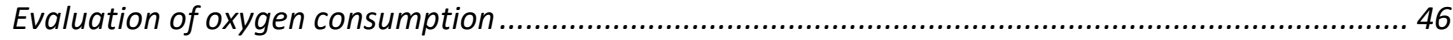

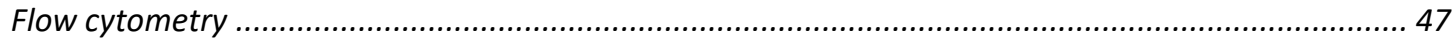

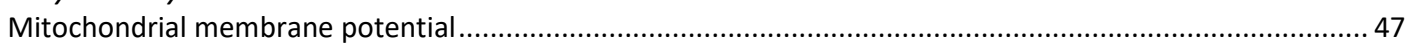

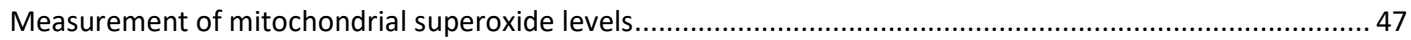

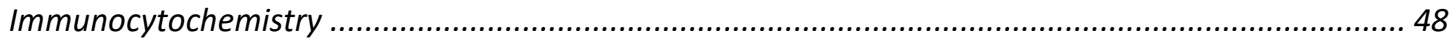

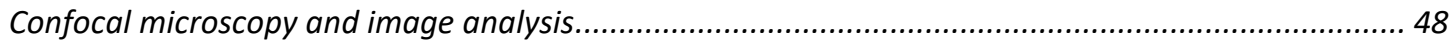

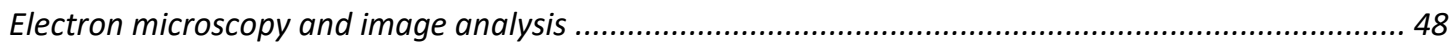

Mitochondrial calcium measurement ....................................................................................... 49

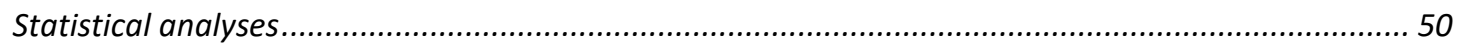

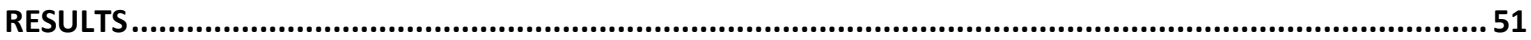

LOSS OF AMPK ACTIVITY RESULTS IN INCREASED ENDOPLASMIC RETICULUM-MITOCHONDRIA CONTACT SITES ....................5 51

Mitochondrial function is compromised in AMPK defective cells .................................................. 51

Absence of AMPK activity enhances ER-mitochondria communication in mouse embryonic fibroblasts

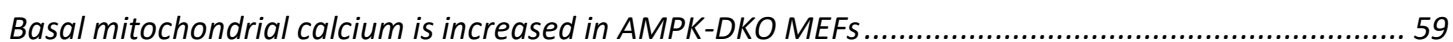

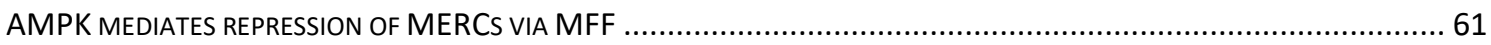

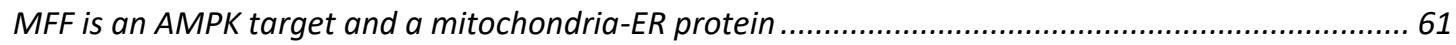

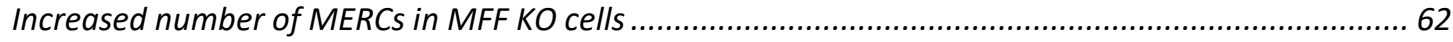

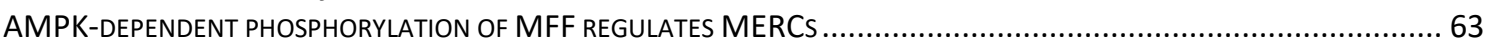

Phosphomimetic MFF Ser ${ }^{155}$ Ser $^{172}$ in AMPK-DKO cells has a gain of function of MFF ...........................63 63

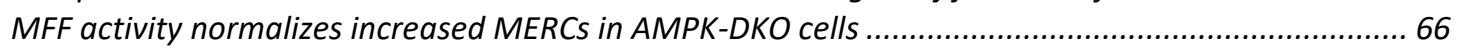

Mitochondrial function is improved with MFF genetic manipulation in AMPK-DKO cells ................... 67

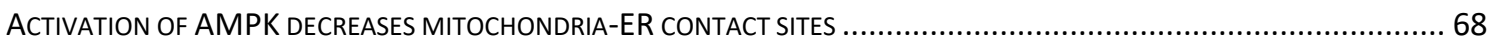

AMPK activation after acute mitochondrial stress decreases the number of mitochondria-ER contact

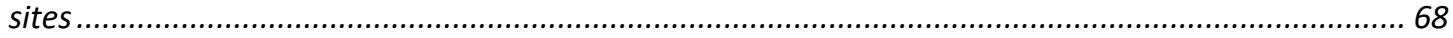

CHRONIC MITOCHONDRIAL RESPIRATORY CHAIN DEFICIENCY RESULTS IN AMPK INHIBITION AND LEADS TO INCREASED

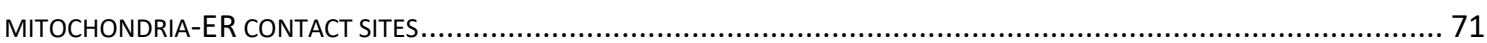

AMPK activity is repressed in a model of chronic mitochondrial stress ............................................ 71

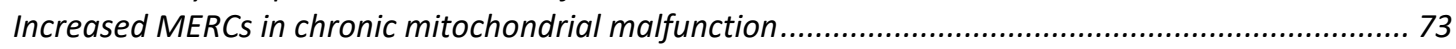

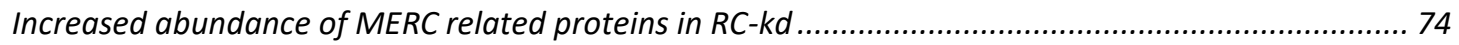

Mitochondrial calcium $\left(\mathrm{Ca}^{2+}\right)$ uptake is enhanced in $\mathrm{RC}$-kd cells.................................................. 76

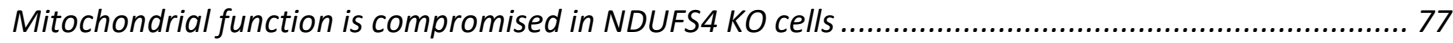

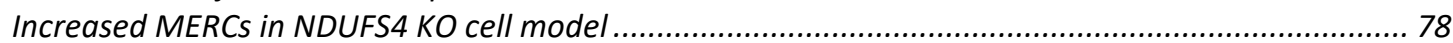

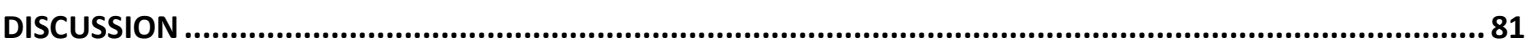

CHRONIC AND ACUTE MITOCHONDRIAL DYSFUNCTION DIFFERENTIALLY REGULATE MERCS ......................................... 81

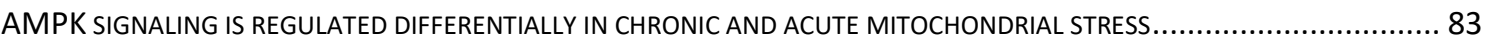

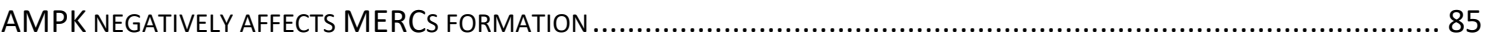

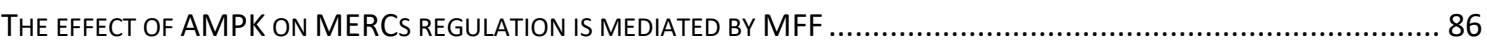

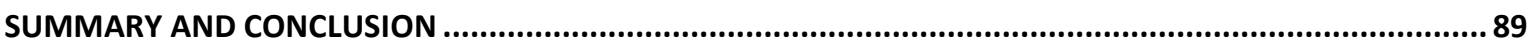

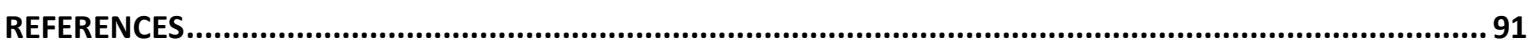

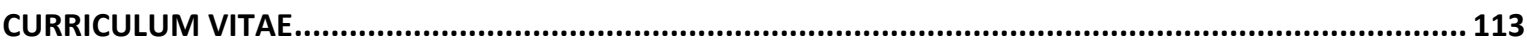




\section{List of figures}

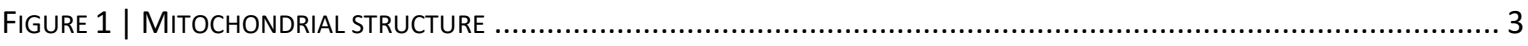

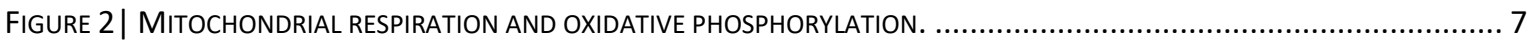

FIGURE 3 | THE DIFFERENT MITOCHONDRIAL-DEPENDENT SIGNALING PATHWAYS. ................................................ 10

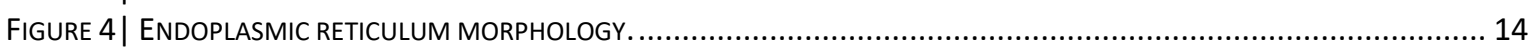

FIGURE 5| ILLUSTRATION OF PROTEINS AND PROTEIN COMPLEXES PRESENT AT ER-MITOCHONDRIA CONTACT SITES (MERCS)... 21

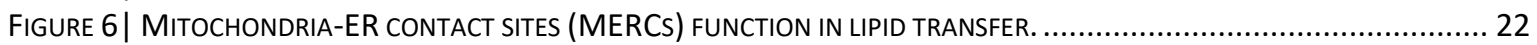

FIGURE 7| CALCIUM (CA2+) TRANSFER OCCURS AT MITOCHONDRIA-ER CONTACT SITES (MERCS)..................................25

FIGURE 8| MITOCHONDRIAL FUNCTION IS COMPROMISED IN A DEFECTIVE AMPK CELL MODEL. ....................................... 52

FIGURE 9| AMPK-DKO CELLS HAVE LOWER MITOCHONDRIAL MEMBRANE POTENTIAL. ............................................... 54

FIGURE 10| MITOSOX FLUORESCENCE IN WT AND AMPK-DKO CELLS, REPRESENTING MITOCHONDRIAL SUPEROXIDE LEVELS. . 55

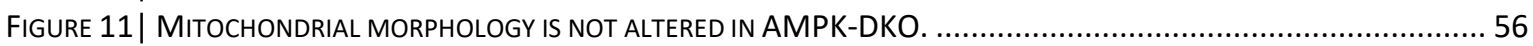

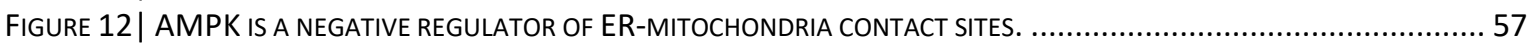

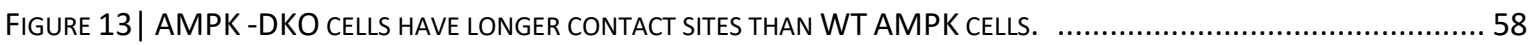

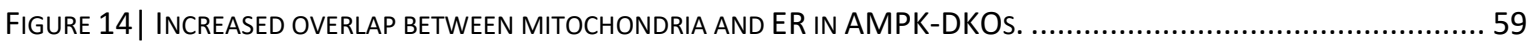

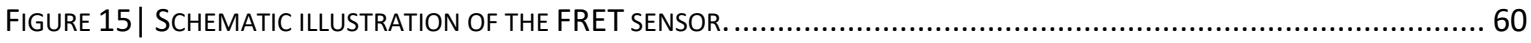

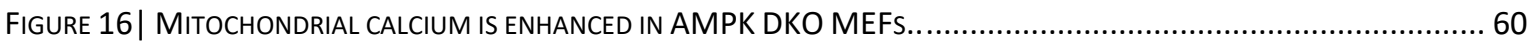

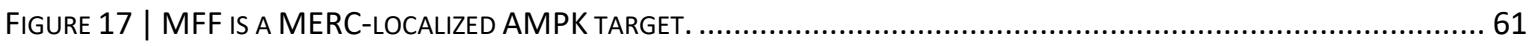

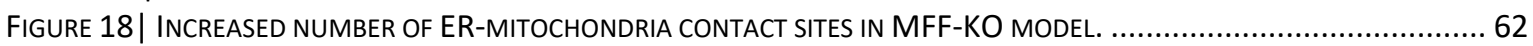

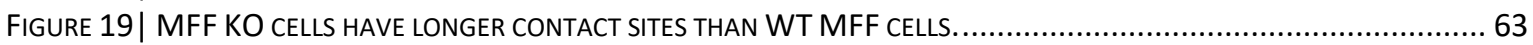

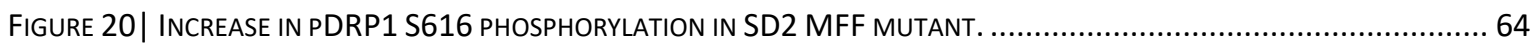

FIGURE 21| MITOCHONDRIAL MORPHOLOGY OF AMPK-DKO EXPRESSING DIFFERENT MFF MUTANTS...............................6 65

FIGURE 22 | AMPK REGULATES THE NUMBER OF ER-MITOCHONDRIA CONTACT SITES VIA MFF. .....................................6. 66

FIGURE 23 | INCREASED EXTENSION OF ER-MITOCHONDRIA CONTACT SITES IN AMPK-DKO EXPRESSING MFF MUTANTS. .......67

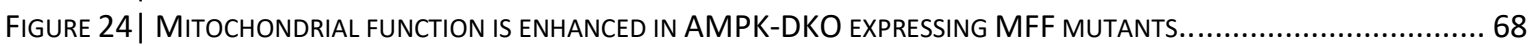

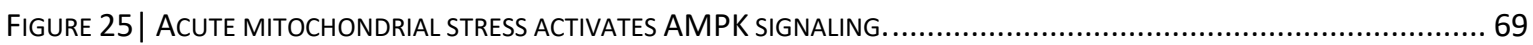

FIGURE 26| ACUTE MITOCHONDRIAL MALFUNCTION DECREASES THE NUMBER OF ER-MITOCHONDRIA CONTACT SITES............ 70

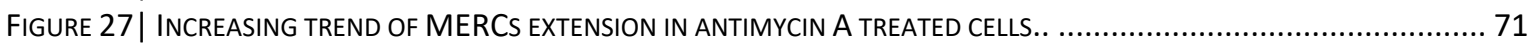

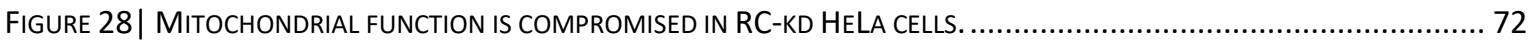

FIGURE 29| INCREASED ER-MITOCHONDRIA CONTACT SITES IN CHRONIC MITOCHONDRIAL DYSFUNCTION............................ 73

FIGURE 30| INCREASED MERC EXTENSION IN CHRONIC MITOCHONDRIAL DYSFUNCTION........................................... 74

FIGURE 31| INCREASED PROTEIN LEVELS OF TETHERS INVOLVED IN MITOCHONDRIA-ER ASSOCIATIONS IN A RC-KD MODEL. ...... 75

FIGURE 32 | INCREASED INTERACTION OF VAPB-PTPIP51 TETHER COMPLEX IN RC-KD HELA CELLS. ................................ 76

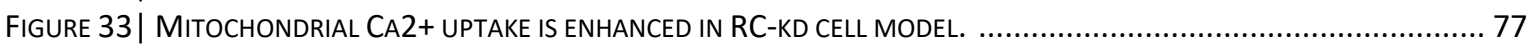

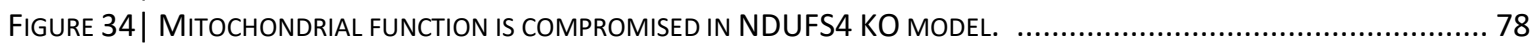

FIGURE 35 | INCREASING TREND OF ER-MITOCHONDRIA CONTACT SITES IN NDUFS4 KO CELL MODEL.............................. 79

FIGURE 36| INCREASED LENGTH OF ER IN CONTACT WITH MITOCHONDRIA IN NDUFS4 KO CELL MODEL. .........................8 80

FIGURE 37|-PROPOSED MODEL OF MITOCHONDRIA-ER COMMUNICATION IN CHRONIC AND ACUTE MITOCHONDRIAL STRESS... 90 


\section{List of tables}

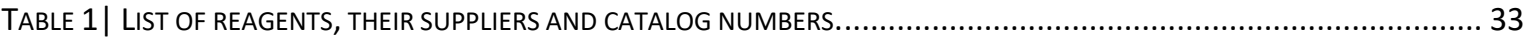

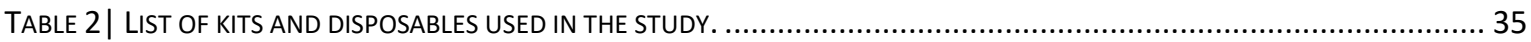

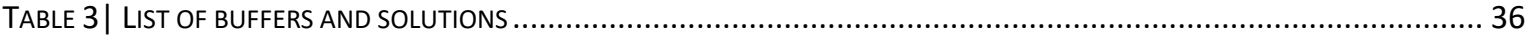

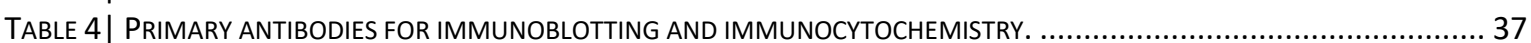

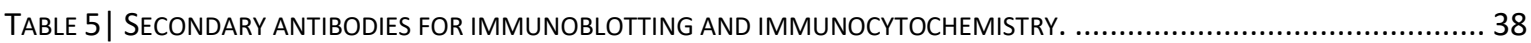

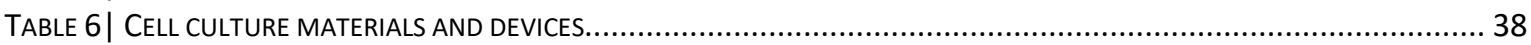

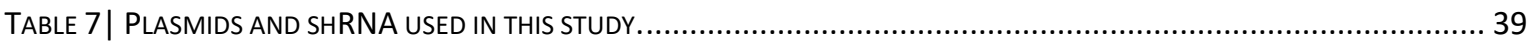

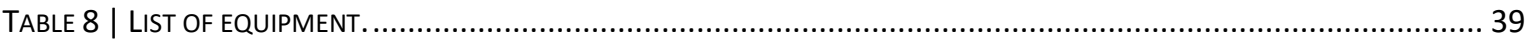

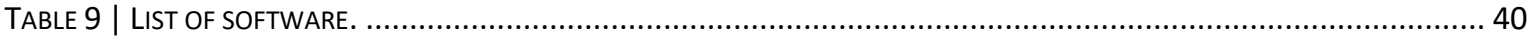




\section{List of abbreviations}

$\Delta \psi \mathrm{m}$

ACBD5

$\mathrm{ACC} 1 / 2$

$A D$

ADP

ALS/FTD

AMPK

APS

ATP

BAP31

BSA

$\mathrm{Ca}^{2+}$

$\mathrm{CICR}$

CLIMP63

CoA

Cyt $C$

DAG

DMEM

DRP1

EM

EMC

ER

ERAD

ERMES

ERRS

ETC

FBS

FCCP

Fis1

FRET

GFP

GPCR

GRP75
Mitochondrial membrane potential

Acyl-coenzyme A binding domain protein 5

Acetyl-CoA carboxylase 1

Alzheimer disease

Adenosine diphosphate

Amyotrophic lateral sclerosis/frontotemporal dementia

Adenosine monophosphate-activated protein kinase

Ammonium Persulfate

Adenosine triphosphate

B-cell receptor-associated protein 31

Bovine serum albumin

Calcium

$\mathrm{Ca}^{2+}$-induced $\mathrm{Ca}^{2+}$ release

63-kDa cytoskeleton-linking membrane protein

Coenzyme-A

Cytochrome $C$

Diacyl-glycerol

Dulbecco's Modified Eagle's Medium

Dynamin-related protein 1

Electron microscopy

ER-membrane protein complex

Endoplasmic Reticulum

ER-associated degradation

ER-mitochondria encounter structure

Estrogens-related receptors

Electron transport chain

Fetal Bovine Serum

Carbonyl cyanide-4-(trifluoromethoxy)phenylhydrazone

Fission 1

Förster/Fluorescence resonance energy transfer

Green fluorescent protein

G protein-coupled receptor

Glucose related regulated protein 75 


\begin{tabular}{|c|c|}
\hline $\mathrm{H}_{2} \mathrm{O}_{2}$ & Hydrogen peroxide \\
\hline IDH & Isocitrate dehydrogenase \\
\hline IMM & Inner mitochondrial membrane \\
\hline IMS & Inner mitochondrial space \\
\hline $\mathrm{IP}_{3}$ & Inositol 1,4,5-triphosphate \\
\hline $\mathrm{IP}_{3} \mathrm{R}$ & Inositol-1,4,5-triphosphate receptor \\
\hline LTPs & Lipid transfer proteins \\
\hline MAMs & Mitochondria-associated membranes \\
\hline MCU & Mitochondrial $\mathrm{Ca}^{2+}$ uniporter \\
\hline MERCs & Mitochondria-ER contact sites \\
\hline MFF & Mitochondrial fission factor \\
\hline MFN1/2 & Mitofusin1/2 \\
\hline MID & Mitochondrial dynamics proteins \\
\hline Mito UPR & Mitochondrial unfolded protein response \\
\hline mPTP & Mitochondrial permeability transition pore \\
\hline mPTP & Mitochondrial permeability transition pore \\
\hline mtDNA & Mitochondrial DNA \\
\hline $\mathrm{O}_{2}$ & Oxygen \\
\hline $\mathrm{O}_{2}^{-}$ & Superoxide anion \\
\hline OCR & Oxygen consumption rate \\
\hline OMM & Outer mitochondrial membrane \\
\hline OPA1 & Optic atrophy 1 \\
\hline ORP & Oxysterol-binding protein (OSBP)-related proteins \\
\hline OSBP & Oxysterol-binding protein (OSBP \\
\hline OsO4 & Osmium tetroxide \\
\hline PACS2 & Phosphofurin acidic cluster sorting protein 2 \\
\hline PC & Phosphatidylcholine \\
\hline PD & Parkinson's disease \\
\hline PDH & Pyruvate dehydrogenase \\
\hline PE & Phosphatidylethanolamine \\
\hline PGC1 $\alpha$ & Peroxisome proliferator-activated receptor- $\gamma$ co-activator $1 \alpha$ \\
\hline $\mathrm{PIP}_{2}$ & Phosphatidylinositol 4,5 biphosphate \\
\hline PLA & Proximity ligation assay \\
\hline PLC & Phospholipase C \\
\hline PM & Plasma membrane \\
\hline
\end{tabular}




\begin{tabular}{|c|c|}
\hline PPAR $\gamma$ & Peroxisome proliferator-activated receptor- $\gamma$ \\
\hline PS & Phosphatidylserine \\
\hline PSS & PS synthase \\
\hline PTPIP51 & Protein tyrosine phosphatase interacting protein 51 \\
\hline$Q$ & Ubiquinone \\
\hline ROS & Reactive Oxygen Species \\
\hline RRBP1 & Ribosome-binding protein 1 \\
\hline rRNAs & Ribosomal ribonucleic acids \\
\hline Rtn4a & Reticulon $4 a$ \\
\hline RyRs & Ryanodine receptors \\
\hline SERCA & Sarcoendoplasmic reticulum $\mathrm{Ca}^{2+}$-ATPase \\
\hline Sig-1R & Sigma-1 receptor \\
\hline SMP & Synaptotagmin-like Mitochondrial lipid-binding \\
\hline SOCE & Store-operated $\mathrm{Ca}^{2+}$ entry \\
\hline SOD2 & Superoxide dismutase 2 \\
\hline SRP & Signal recognition particle \\
\hline STIM1 & Stromal interaction molecule 1 \\
\hline SYNJ2BP & Synaptojanin-2 binding protein \\
\hline TAC & Tip attachment complex \\
\hline TCA & Tricarboxylic acid \\
\hline TDP-43 & Tar DNA-binding proteins- 43 \\
\hline TEM & Transmission electron microscopy \\
\hline tRNAs & Transfer ribonucleic acids \\
\hline UA & Uranyl acetate \\
\hline UPR & Unfolded protein response \\
\hline VAPB & Vesicle-associated membrane protein-associated protein B \\
\hline VDAC & Voltage-dependent anion channel \\
\hline VPS13A & Vacuolar protein sorting-associated protein \\
\hline
\end{tabular}




\section{ABSTRACT}

In eukaryotic cells, inter-organelle communication is crucial for several cellular functions, as well as for several cell signaling mechanisms. Mitochondria and the endoplasmic reticulum (ER), for example, form tight contact sites that are implicated in many aspects of cell physiology, and whose disruption has been associated with pathology, particularly neurodegenerative diseases. Although the contacts between these organelles are one of the most studied and most stable, the basic understanding of their regulation, as well as the communication of these two organelles via signaling pathways remains unclear.

Here, we explored the regulatory environment of the mitochondria-ER contact sites (MERCs) in various mammalian cells with mitochondrial malfunction. To identify the mechanisms regulating mitochondria and ER crosstalk, we used cellular models of acute and chronic mitochondrial dysfunction, which were generated by chemical inhibition of the respiratory chain and by silencing the expression of UQCRC1 in HeLa cells or preparing murine embryonic fibroblasts from the Ndufs4 KO mouse.

Interestingly, we found distinct effects of mitochondrial malfunction on MERCs formation depending on whether the mitochondrial defect was acute or chronic. Electron microscopy (EM) analysis showed a high number of MERCs in both UQCRC1 KD and Ndufs4 KO models of chronic mitochondrial malfunction. Opposed to these data, acute mitochondrial stress seems to repress MERCs formation, since fewer contact sites were observed. Importantly, we found differential response of AMPK to chronic and acute mitochondrial dysfunction, which negatively correlated with MERCs formation. Indeed, while chronic mitochondrial malfunction resulted in the repression of AMPK, acute mitochondrial stress activated AMPK signaling. Consistently, in AMPK depleted cells, EM and confocal analyses confirmed the increased mitochondria-ER juxtaposition, which was observed in the chronic mitochondrial malfunction models.

We also identified mitochondrial fission factor (MFF), which is involved in mitochondrial fission, as an AMPK target that localized to the MERCs. Indeed, we showed that MFF is involved in the regulation of MERCs, since a robust increase in the number of MERCs was observed in cells lacking MFF. Furthermore, a phospho-mimetic isoform of MFF normalized the number of MERCs to WT levels in cells lacking AMPK activity, suggesting that AMPK mediates the repression of mitochondria-ER contact sites via phosphorylation of MFF. 
Taken together, our data identified a cellular signaling pathway downstream of a key kinase, AMPK that regulates the number of MERCs by modulating MFF activity. Moreover, our results highlight that the association of mitochondria with ER is yet another aspect of mitochondrial biology under the control of AMPK. This study contributed to a better understanding of MERCs by elucidating their response to mitochondrial respiratory chain malfunction in a defined manner, thereby providing a better understanding of pathological conditions linked to MERCs. 


\section{INTRODUCTION}

\subsection{Mitochondria}

\subsubsection{Mitochondrial Structure and function}

In eukaryotic cells, the mitochondrion is the organelle responsible for aerobic respiration. This organelle is a double membrane-enclosed sub-compartment of the cell, which uses oxygen to produce energy, in the form of Adenosine triphosphate (ATP), and thus has been considered for many years as the "powerhouse" of the cells. The mitochondrion is an example of endosymbiont from the endosymbiosis theory, which defends that the inner mitochondrial membrane (IMM) and their content is derived from an ancestral bacteria (Sagan, 1967). In fact, it was discovered that these organelles carry their own DNA (mtDNA). The mitochondrial genome codes 37 genes comprising 13 proteins linked to respiratory chain complexes, 2 ribosomal RNAs (rRNAs) and 22 transfer RNAs (tRNAs). Mitochondria have a very complex ultrastructure, composed of two distinct membranes, the outer mitochondrial membrane (OMM) and the IMM (Figure 1).

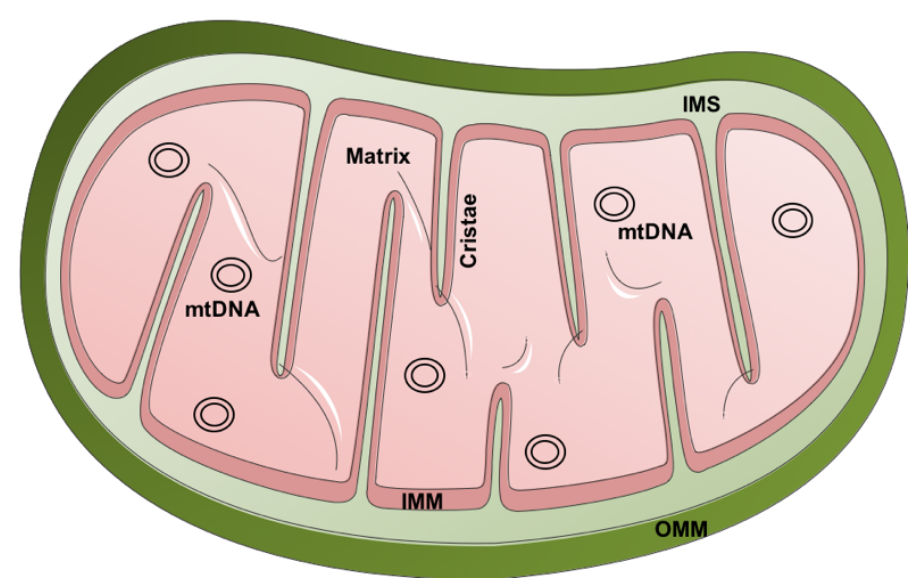

Figure 1 | Mitochondrial structure. Scheme of a mitochondrion showing the outer mitochondrial membrane (OMM) surrounding the intermembrane space (IMS) and inner mitochondrial membrane (IMM). The folding of IMM generates the cristae, which encloses the mitochondrial matrix, where the respiratory chain complexes and mtDNA are located.

The OMM acts as a barrier between the cytoplasm and the intermembrane space (IMS). This barrier is important to avoid the entry of small molecules, as well as to protect against some deleterious mitochondrial factors, such pro-apoptotic factors. In addition, voltage-dependent anion channel (VDAC) is located at the OMM, and is responsible for the entrance of some ions (e.g. $\mathrm{Ca}^{2+}$ ) (Colombini, 1980; Zalman et al., 1980). 
The respiratory chain complexes and carriers for metabolites are located in the IMM. An important characteristic of this membrane is the presence of cardiolipin, a required phospholipid for optimal mitochondrial respiratory chain performance. Moreover, IMM is recognized by the presence of invaginations, called cristae (Figure 1). The cristae are the key structural components in the mitochondria, in which the respiratory chain and the OXPHOS machinery are located. The mitochondrial matrix is where processes involved in mitochondrial metabolism, and maintenance, replication, transcription and translation of mtDNA occur (Scalettar et al., 1991).

Besides ATP production, mitochondria have been also associated with essential cellular functions, such as amino acid and fatty acid metabolism, iron-sulfur cluster biogenesis, heme and steroid synthesis, and calcium homeostasis (Nunnari and Suomalainen, 2012). Mitochondria are also involved in the regulation of cellular processes, like autophagy and apoptosis, cell proliferation and differentiation (Nunnari and Suomalainen, 2012; Raimundo, 2014). Due to the pivotal importance of mitochondria in cellular physiology, mitochondrial malfunction is implicated in several pathologies (DiMauro and Schon, 2008). For instance, it has been shown that defects in genes encoding mitochondrial proteins result in disease, including myopathies and neurodegenerative diseases (DiMauro and Schon, 2008).

\subsubsection{Mitochondrial metabolism}

In eukaryotic cells, most of the energy is derived from oxidation of carbohydrates and fatty acids in mitochondria because the oxidation of glucose via glycolysis is not so efficient. Therefore, several metabolic pathways take place in mitochondria, where enzymes located in the IMM or the matrix are involved.

\subsubsection{Glycolysis}

Glycolysis is the process in which glucose is oxidized to pyruvate, and cytosolic enzymes are responsible for this process. The pathway is composed of 10 reactions, and it generates pyruvate and NADH in the cytosol. Two net ATP molecules per glucose molecule are generated in this metabolic pathway. The pyruvate generated can then enter into the mitochondrial matrix under aerobic conditions, where is metabolized to acetyl-Coenzyme $A(\mathrm{CoA})$, thus feeding the tricarboxylic acid (TCA) cycle and the downstream aerobic metabolism. However, in the absence of oxygen $\left(\mathrm{O}_{2}\right)$, lactate is formed through NADH oxidation by pyruvate. 


\subsubsection{Tricarboxylic acid cycle}

Franz Koop and Carl Martinus made some of the first contributions to the discovery of the TCA cycle, where they found a reaction to form citrate from oxaloacetate. However, it was in 1937 that Hans Krebs and William Johnson discovered the reaction sequence of the TCA cycle, from citrate oxidation to oxaloacetate. In addition, they proposed a two-carbon donor, which allowed the conversion of oxaloacetate to citrate (Krebs and Johnson, 1937). The TCA cycle occurs in the mitochondrial matrix, where the enzymes responsible for this pathway are located, with the exception of succinate dehydrogenase, which is localized to the matrix side of the IMM. AcetylCoA is considered the bridge between the end product of glycolysis, pyruvate, and the synthesis of citrate; therefore acetyl-CoA is the "initial" substrate of TCA cycle. However, it is also possible to obtain acetyl-CoA from other sources, such as beta-oxidation of fatty acids, and from the catabolism of the amino acids, lysine, leucine, phenylalanine, tyrosine and tryptophan.

The pyruvate generated from glycolysis is transported into mitochondria through the pyruvate carrier, where it undergoes oxidative decarboxylation by the pyruvate dehydrogenase (PDH) complex, resulting in acetyl-CoA as product. The TCA cycle starts by the acetyl-CoA reacting with oxaloacetate to form citrate, which is catalyzed by citrate synthase (Stern and Ochoa, 1949; Stern et al., 1952). The next reaction is catalyzed by aconitase, which converts citrate to isocitrate. The first oxidative decarboxylation of the cycle occurs in the synthesis of $\alpha$-ketoglutarate from isocitrate, and it is catalyzed by isocitrate dehydrogenase (IDH), in which one mole of $\mathrm{NAD}^{+}$is reduced to $\mathrm{NADH}$, and one mole of $\mathrm{CO}_{2}$ is released, per mole of $\alpha$-ketoglutarate produced (Adler et al., 1939; Plaut and S. Sung, 1954). In the next step, there is the conversion of $\alpha$-ketoglutarate to succinyl-CoA, catalyzed by the $\alpha$-ketoglutarate dehydrogenase complex, resulting in $\mathrm{CO}_{2}$ release, and in the reduction of one mole of $\mathrm{NAD}^{+}$to $\mathrm{NADH}$. It is in this step that the decarboxylation stages of the TCA cycle terminate, giving way to the next steps that are characterized by several oxidation reactions from succinate in order to recover oxaloacetate. Succinyl-CoA is converted to succinate by succinyl-CoA synthetase, generating GTP /ATP and COA-SH. The sixth reaction of the TCA cycle is characterized by the oxidation of succinate to fumarate, and the reduction of FAD to FADH 2 . This reaction is catalyzed by succinate dehydrogenase, also known as complex II of respiratory chain. As such, this is a direct contact point between the TCA cycle and the respiratory chain. Fumarate hydratase is responsible for the reversible hydration of fumarate to malate. Finally, in the last reaction of the TCA cycle, where oxaloacetate is recovered from malate, catalyzed by malate dehydrogenase, in which there is oxidation of malate with concomitant reduction of NAD+. 


\subsubsection{The respiratory chain and oxidative phosphorylation}

The electron transport chain (ETC), also called mitochondrial respiratory chain, is composed of a group of protein complexes present in the IMM, which is also the place where the ATP synthase is localized. This is a very efficient pathway that couples ATP generation to the oxidation of NADH and $\mathrm{FADH}_{2}$. The ETC consists of complex I, complex II, complex III and complex IV. The complex V, also referred to as mitochondrial ATP synthase, consists of a multi-complex structure made of $F_{1}$ and $F_{0}$ subunits, and is able to transform adenosine diphosphate (ADP) to ATP (Mitchell and Moyle, 1969; Scheffler, 2008). The ETC is responsible for the electron transfer from electron donors to electron acceptors via redox reactions. Additionally, there is pumping of $\mathrm{H}^{+}$across the IMM, from the matrix to the IMS, generating an electrochemical gradient across the IMM, which is required for the process of ATP synthesis via oxidative phosphorylation (MITCHELL, 1961). Briefly, in complexes I and II, NADH and succinate $\left(F A D H_{2}\right)$ are oxidized to $N A D^{+}$and $F A D$ respectively. The electrons released from both complexes are directed to an electron transporter, ubiquinol, which in turn carries the electrons to complex III. In complex III, the received electrons are transferred via ubiquinone cycle to cytochrome $\mathrm{c}$, the second electron transporter in ETC. Cytochrome $\mathrm{c}$ gives the electrons to complex IV (cytochrome c oxidase), which are then passed to $\mathrm{O}_{2}$, forming $\mathrm{H}_{2} \mathrm{O}$. These redox reactions are able to release energy, which is used by complex I, III and IV to pump $\mathrm{H}^{+}$into the IMS. However, these protons are returned to the matrix through ATP synthase (complex V), promoting ATP synthesis from the phosphorylation of ADP and Pi (Figure 2) (Scheffler, 2008).

The crucial role of ETC in the cellular metabolism is known, being the major source of ATP production. As a result, some compounds which can interfere with the complexes of ETC, inhibiting them and consequently impairing the normal functioning of oxidative phosphorylation have been described. These compounds are crucial for understanding further the role of the ETC in several cellular processes. For instance, complex I can be inhibited by a specific inhibitor, rotenone, preventing ubiquinone reduction. However, complex II remains functional, and by transferring electrons to complex III, it allows the functioning of the ETC, although to a lower extent of electron flow. Antimycin A inhibits complex III, and therefore makes it virtually impossible to transfer electrons coming from complexes I and II to the final acceptor, $\mathrm{O}_{2}$. Cyanide is the most potent inhibitor of the ETC, and binds irreversibly to complex IV, making it impossible for $\mathrm{O}_{2}$ to bind to complex IV. Moreover, there is oligomycin which inhibits the phosphorylation in complex $\mathrm{V}$, blocking the $F_{0}$ subunit of ATP synthase. This inhibition stops the entrance of protons from the IMS into the matrix and consequently blocks ATP production (Figure 2). 
Other notable compounds are the uncouplers of respiratory chain, for example carbonyl cyanide4-(trifluoromethoxy)phenylhydrazone (FCCP), which are fatty acids that are able to associate with the protons in the IMS and transport them to the mitochondrial matrix (Kessler et al., 1976; Lou et al., 2007). These uncouplers increase the ETC velocity, allowing an increased reduction of $\mathrm{O}_{2}$ to $\mathrm{H}_{2} \mathrm{O}$, but no ATP is generated due to the dissipation of electrochemical gradient. Thus, these agents uncouple the respiratory chain from oxidative phosphorylation (Lou et al., 2007; Terada, 1990).

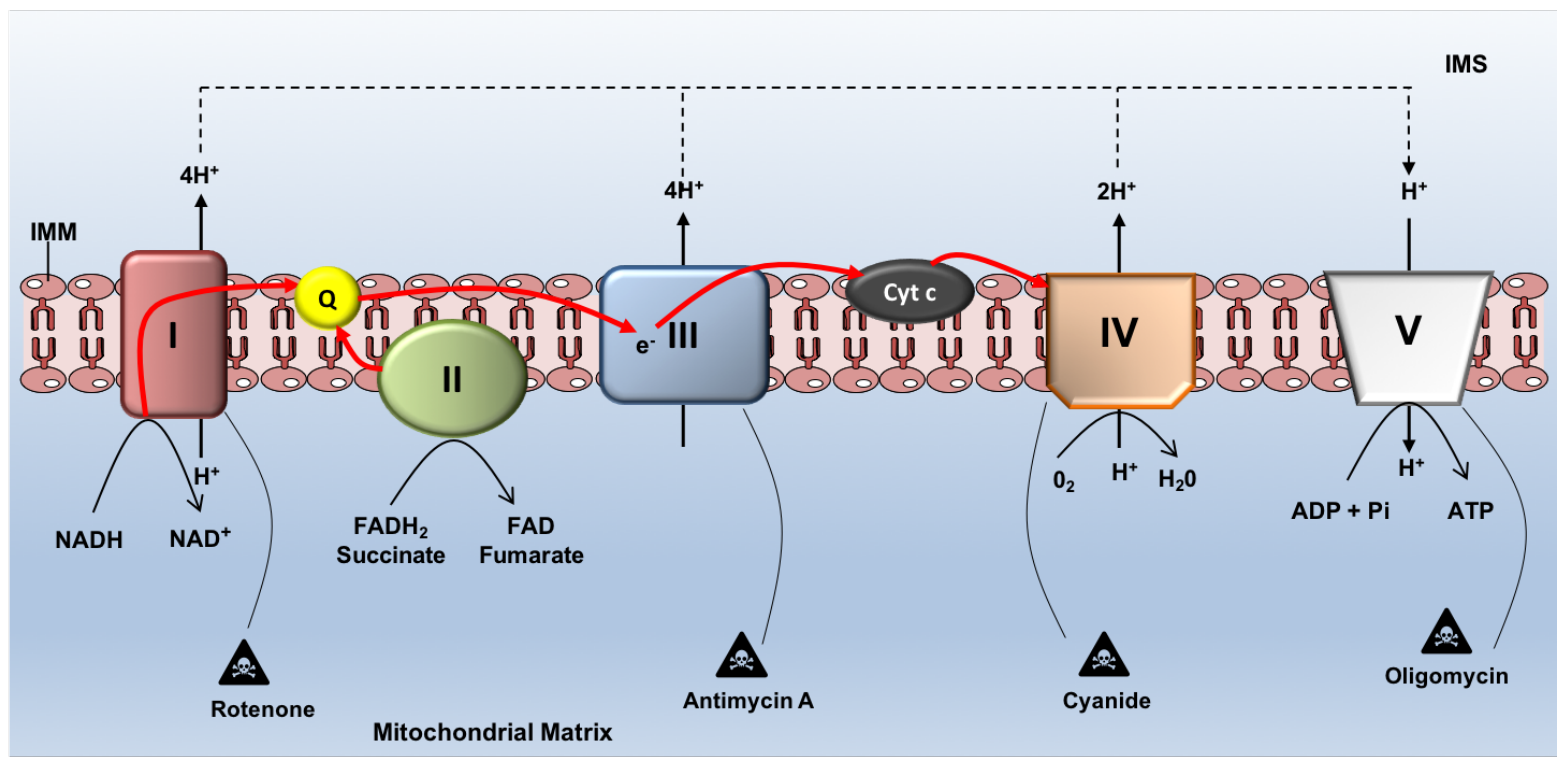

Figure 2 | Mitochondrial respiration and oxidative phosphorylation. The respiratory chain complexes (I-IV), ubiquinone (Q), cytochrome c (Cyt c) and ATP synthase (complex V) are located in the inner mitochondrial membrane (IMM). Red arrows represent the transfer of electrons between respiratory chain complexes.

\subsubsection{Mitochondrial dynamics}

Mitochondria are not anymore considered as static, rod-shaped organelles; nowadays they are very well known to undergo processes like fusion and fission. These two concepts were already discovered in 1914 by Lewis and Lewis, using chick embryo cells in culture (LEWIS and LEWIS, 1914). The division of one mitochondrion into two daughter mitochondria is a process called mitochondrial fission. On the other hand, mitochondrial fusion is characterized by the union of two mitochondria (Tilokani et al., 2018). A balance between fission and fusion is necessary in order to regulate mitochondrial morphology, distribution and function (Chan, 2012). Moreover, these mitochondrial parameters can change depending on cell type, and on the specific needs of the cell. It is known that optimal mitochondrial performance is obtained with a healthy mitochondrial 
morphology. Therefore, mitochondrial dynamics is essential in mitochondrial quality control (Chan, 2012; Pernas and Scorrano, 2016). Due to deregulation of these two processes, mitochondria can have a fragmented profile, which is characterized by a large number of small round-shape mitochondria or a hyperfused network observed as elongated and well connected mitochondria (Tilokani et al., 2018). Naturally, some pathological conditions are associated with these different mitochondrial morphology profiles (Nunnari and Suomalainen, 2012).

The process of mitochondrial fission requires some proteins and one key player is a member of the dynamin superfamily of GTPases, dynamin-related protein 1 (DRP1) (Smirnova et al., 2001). DRP1 is a cytosolic protein, which during mitochondrial division is recruited to mitochondria by adaptor proteins, such as, Fission 1 (FIS1), mitochondrial fission factor (MFF), mitochondrial dynamics proteins (MiD) of 49kDa (MiD49) or 51kDa (MiD51) (Gandre-Babbe and van der Bliek, 2008; Losón et al., 2013; Palmer et al., 2013). The recruitment of DRP1 to the OMM allows its oligomerization into a ring-like structure contributing to membrane constriction and scission in a GTP-dependent manner (Fonseca et al., 2019; Ingerman et al., 2005; Kamerkar et al., 2018; Kraus and Ryan, 2017; Labrousse et al., 1999; Yoon et al., 2001). In this process, the recruitment of DRP1 by adaptor proteins is essential, due to the inability of DRP1 to bind membrane phospholipids by itself because it lacks a PHD domain (Tilokani et al., 2018). Furthermore, it is known that overexpression of MFF enhances mitochondrial fragmentation, while mitochondrial elongation following MFF genetic silencing has been demonstrated (Otera et al., 2010). Mitochondrial fission is associated with cellular processes, such as apoptosis and mitochondrial proliferation during cell division (Chan, 2012; Pernas and Scorrano, 2016).

Mitochondrial fusion is carried out by mitofusins 1 and 2 (MFN1 and MFN2 respectively) and optic atrophy 1 (OPA1). The latter is responsible for IMM fusion, while MFN1 and MFN2 mediate the OMM fusion (Pernas and Scorrano, 2016). Therefore, mitochondrial fusion requires two different events, OMM and IMM fusion. Indeed, the overexpression of mitofusins is associated with mitochondrial aggregation around the nucleus (Eura, 2003). On the other hand, their knockout induces severe mitochondrial morphology defects leading to embryonic lethality in mice and embryonic fibroblasts (Chen et al., 2003; Davies et al., 2007; Ishihara et al., 2009; Wakabayashi et al., 2009).

During mitochondrial fusion, MFN1 and MFN2, two large GTPases, tether two closely apposed mitochondria by establishing homo or heterotypic complexes in trans between both proteins via their HR2 domains, leading to mitochondrial fusion (Chen et al., 2003; Koshiba, 2004) (Hoppins et 
al., 2011). However, another model of the tethering was proposed recently, in which the proteins interact via GTPase domains (Cao et al., 2017; Qi et al., 2016). As a consequence of GTP binding and/or hydrolysis, a conformational change of mitofusins occurs, promoting mitochondrial docking. Finally, the OMM fusion is ensured by GTPase-dependent power stroke or GTP-dependent oligomerization (Tilokani et al., 2018). As mentioned above, IMM fusion is required to complete the mitochondrial fusion process, and is mediated by another GTPase, OPA1, and specific lipids present in the IMM (Tilokani et al., 2018). It has been demonstrated that OPA1 and cardiolipin interact after OMM fusion, contributing to IMM fusion. This interaction allows the tethering of both IMM, which in the end fuse as a result of GTP hydrolysis (Ban et al., 2017; Tilokani et al., 2018). Mitochondrial fusion has been shown to be essential, improving cell survival due to the ability to form branches and interconnected organelles, which in turn allows the exchange of IMS and matrix contents, including mtDNA (Gomes et al., 2011a, 2011b; Rambold et al., 2011).

\subsubsection{Mitochondria and signaling pathways}

Nowadays, it is clear that mitochondria do not only play a role in metabolic functions but also they have signaling functions by communicating their biosynthetic and bioenergetic performance to the rest of the cell (Chandel, 2014). The idea that mitochondria communicate with the cytosol was raised in the 1990s by several groups, and currently, it is well known that mitochondria have several mechanisms to communicate their performance. Some examples are the release of metabolites and ROS, activation of $5^{\prime}$ adenosine monophosphate-activated protein kinase (AMPK), release of peptides, as well as changes in inner mitochondrial membrane potential and calcium (Chandel, 2015). It is possible that all these processes cooperate together, because once mitochondrial respiratory chain flux is impaired, several other consequences appear, such as decrease of ATP release, and a decline of ROS and TCA cycle metabolites can also be observed (Chandel, 2015; Raimundo et al., 2011). Several diseases have been associated with defects of mitochondrial metabolism and consequent impairment to generate ATP, causing mitochondrial disorders, particularly in cells with high energetic demand, such as neurons or cardiomyocytes. Nevertheless, the connection between mitochondrial signaling and mitochondrial impairment is now known. Besides the release of cytochrome $C$ from mitochondria to initiate apoptosis or ROS to induce hypoxic gene expression (Chandel et al., 2000; Liu et al., 1996; Raimundo, 2014), there are other signaling pathways that are able to connect mitochondria to the cytosol, and to the nucleus, such as AMPK signaling, mitochondrial unfolded protein response (mito ${ }^{\mathrm{UPR}}$ ) or calcium $\left(\mathrm{Ca}^{2+}\right)$ release (Figure 3) (Cereghetti et al., 2008; Pellegrino et al., 2013; Raimundo, 2014; Rizzuto et al., 2012). 
Several other mitochondrial-related pathways are known, but only those, which are relevant in the context of this thesis, will be discussed further.

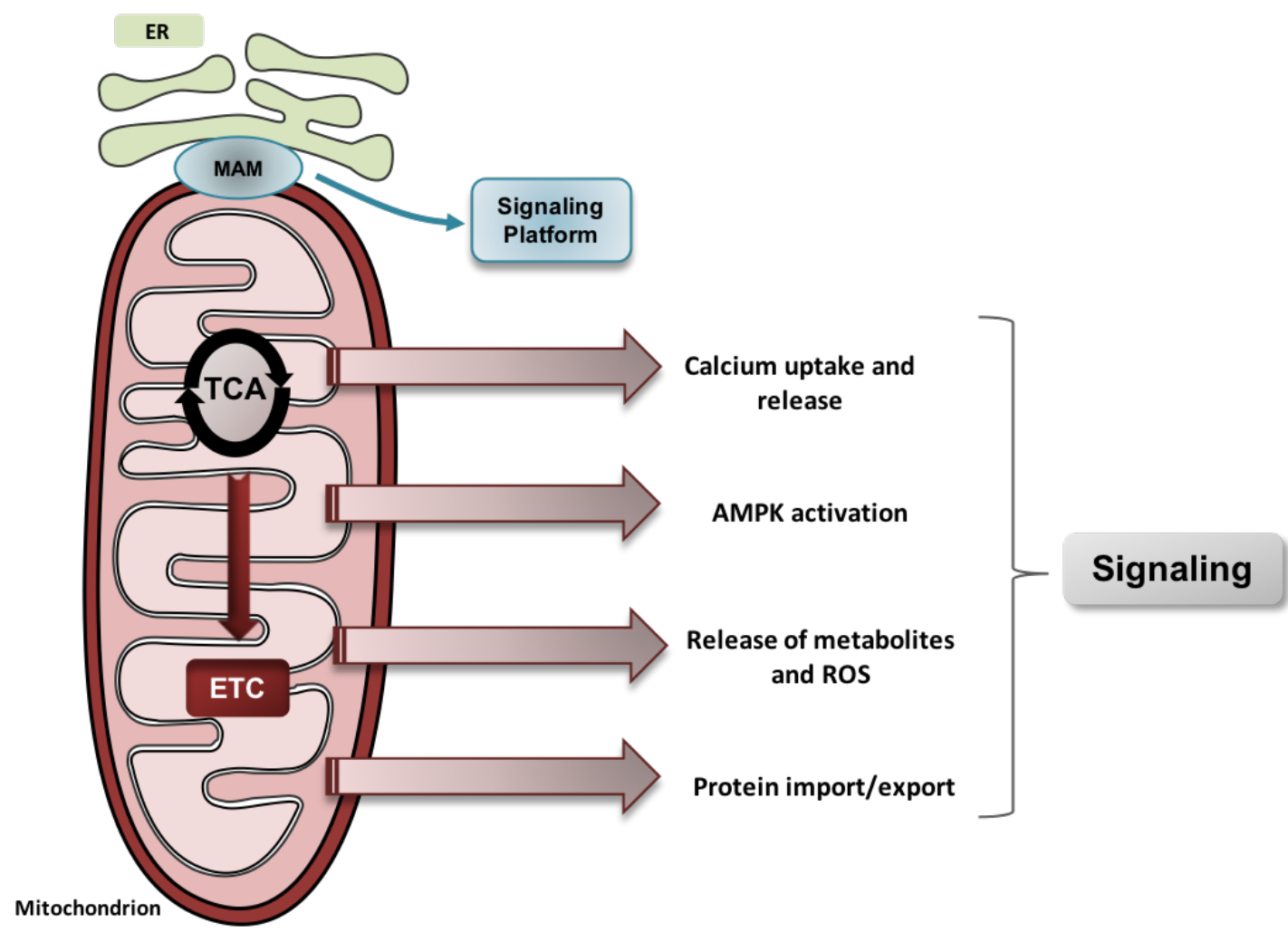

Figure 3 | The different mitochondrial-dependent signaling pathways. In eukaryotes, mitochondria have a role in signaling pathways, in order to communicate mitochondrial fitness to the rest of the cell. Included in these signaling pathways are AMPK activation, release of metabolites and reactive oxygen species (ROS), and also calcium uptake and release. Moreover, MAMs are a greater signaling platform. ETC, electron transport chain; TCA, tricarboxylic acid; MAM, mitochondria-associated membranes; ER, endoplasmic reticulum.

Mitochondria are one of the sources of ROS in the cells, and they are generated by complexes I and III of the respiratory chain (Murphy, 2009). The superoxide anion $\left(\mathrm{O}_{2}^{-}\right)$released to the mitochondrial matrix and to the IMS is converted to $\mathrm{H}_{2} \mathrm{O}_{2}$ by the enzymes superoxide dismutase 2 (SOD2) and SOD1, respectively (Murphy, 2009). Nowadays, ROS are not only considered as toxic by-products in the cell, but they are also molecules that can regulate signaling pathways from mitochondria to the rest of the cell (Murphy, 2009). Indeed, $\mathrm{H}_{2} \mathrm{O}_{2}$ can be considered as a good mitochondrial signal since it crosses membranes (Han et al., 2003). Furthermore, the role of mitochondrial ROS in the regulation of proliferation and differentiation has been shown (Nunnari and Suomalainen, 2012). Additionally, a decrease in the activation of signaling pathways required for cell proliferation, differentiation, and metabolic adaptation was observed when the levels of ROS were reduced (Sena 
and Chandel, 2012). Moreover, ROS have been associated with the regulation of cellular adaptation to hypoxia, due to release of mitochondrial ROS stimulated by hypoxia conditions (Chandel et al., 1998). In 2012, Raimundo et al. showed ROS-dependent activation of AMPK, with consequent apoptosis mediated by E2F1 (Raimundo et al., 2012) further highlighting the widespread nature of mitochondrial ROS signaling in the cell.

In addition to ROS, AMPK signaling can be activated after a drop in ATP levels, with a concomitant increase of AMP levels. This results in a switch from an anabolic to a catabolic process by the phosphorylation of key proteins in multiple pathways, including mitochondrial homeostasis (Egan et al., 2011a; Herzig and Shaw, 2018; Jager et al., 2007; Toyama et al., 2016). This mechanism is considered important for cells to restore cellular energy supply during energy stress.

One mechanism of AMPK action is the stimulation of the breakdown of macromolecules, such as mobilization of lipid stores, to generate energy (Herzig and Shaw, 2018). After stimulation of lipases and release of fatty acids from triglyceride stores, free fatty acids are imported into mitochondria for $\beta$-oxidation (Ahmadian et al., 2011). Moreover, AMPK activity modulates the activity of the acetyl transferase, CPT1, which is responsible for the transport of fatty acids into mitochondria (Herzig and Shaw, 2018). In addition, acetyl-CoA carboxylase 1 (ACC1) and ACC2, targets of AMPK, are able to produce malonyl-CoA, which in turn can inhibit CPT1 (McGarry et al., 1978; Saggerson, 2008). Therefore, phosphorylation and inhibition of ACC by AMPK contributes to reduced levels of malonyl-CoA, promoting fatty acid import to mitochondria for $\beta$-oxidation (Herzig and Shaw, 2018). Another mechanism of AMPK activity in mitochondrial signaling is the upregulation of genes involved in mitochondrial biogenesis, thus controlling mitochondrial number. There are several downstream effectors of AMPK that contribute to mitochondrial biogenesis, including peroxisome proliferator-activated receptor- $\gamma$ co-activator $1 \alpha(\mathrm{PGC1} \alpha)$, which in turn is able to interact with peroxisome proliferator-activated receptor- $\gamma$ (PPAR $\gamma$ ) or estrogen-related receptors (ERRs), thereby activating mitochondrial biogenesis (Garcia-Roves et al., 2008; Herzig and Shaw, 2018; Jager et al., 2007).

An important aspect of mitochondria biology that AMPK regulates is the shape of the mitochondrial network. It is known that mitochondrial insults, including ETC inhibition, are associated with mitochondrial fragmentation (Wai and Langer, 2016). Interestingly, these inhibitors of ETC are also able to activate AMPK, which was shown to be required for mitochondrial fragmentation after rotenone or antimycin A treatment (Toyama et al., 2016). Moreover, in the same study, the receptor of DRP1 in the OMM, MFF, was discovered as an AMPK substrate, responsible for mitochondrial constriction in the mitochondrial fission process (Ingerman et al., 2005; Kraus and 
Ryan, 2017; Toyama et al., 2016). Additionally, an increase of DRP1 at mitochondria was observed after AMPK activation and MFF phosphorylation (Toyama et al., 2016).

Finally, AMPK is also able to regulate autophagy by regulating some aspects of the autophagy machinery in mammalian cells and in yeast (Herzig and Shaw, 2018). It was shown in 2011 that AMPK phosphorylates ULK1, an important kinase for autophagy activation (Egan et al., 2011a; Kim et al., 2011). Indeed mitochondrial respiratory chain deficiency was recently shown to regulate, through AMPK signaling, the biogenesis of lysosomes, which are crucial organelles for the autophagic process (Fernández-Mosquera et al., 2017) suggesting that mitochondria play a role in the autophagy process via AMPK activation.

An important aspect of mitochondrial signaling is its involvement in $\mathrm{Ca}^{2+}$ signaling. Moreover, ATP production is regulated by $\mathrm{Ca}^{2+}$ signaling, since this cation can enter into the mitochondrial matrix and regulate proteins, enzymes and transporters, which are involved in ATP production (Rossi et al., 2019). Mitochondrial $\mathrm{Ca}^{2+}$ uptake is dependent on the electrochemical gradient across the IMM generated by the respiratory chain (Rizzuto et al., 2012). In the OMM, VDAC allows the entrance of $\mathrm{Ca}^{2+}$; however, the IMM is impermeable to this cation, requiring specialized channels such as the mitochondrial $\mathrm{Ca}^{2+}$ uniporter (MCU), which is responsible for $\mathrm{Ca}^{2+}$ entrance into mitochondria (Baughman et al., 2011; De Stefani et al., 2011). Additionally, mitochondria are also able to export $\mathrm{Ca}^{2+}$ through the $\mathrm{Na}^{+} / \mathrm{Ca}^{2+}$ exchanger (Rossi et al., 2019).

It is important to note that although mitochondrial $\mathrm{Ca}^{2+}$ can be beneficial, and can regulate mitochondrial metabolism, on the other hand, it can also be toxic, especially under conditions of high amounts of $\mathrm{Ca}^{2+}$ in the matrix, thus contributing to pathological conditions (Rossi et al., 2019). It is very well established that $\mathrm{Ca}^{2+}$ overload is responsible for the induction of cell death by apoptosis or necrosis. Mitochondrial $\mathrm{Ca}^{2+}$ in excess is able to open the mitochondrial permeability transition pore (mPTP), promoting the release from mitochondria of cytochrome $c$, which is a proapoptotic factor that has been implicated in apoptotic cascade initiation (Rasola and Bernardi, 2011). 


\subsection{Endoplasmic reticulum}

\subsubsection{Endoplasmic reticulum structure}

In 1945, scientists observed for the first time by electron microscopy a "lace-like" structure in the cells. However, it was only in 1952 that Porter and Kallman introduced the term endoplasmic reticulum (ER), where the preferential localization of vesicular structures in the perinuclear area of the cytoplasm was observed (Palade, 1956).

The mammalian ER is characterized by continuous membrane structures, which are organized into different subdomains, as nuclear envelope and peripheral ER. The latter subdomain is defined as flat sheets and branched tubules, which includes the rough ER and smooth ER, respectively (Figure 4) (Park and Blackstone, 2010; Schwarz and Blower, 2016). However, in the presence of cellular stresses, ER architecture can be modified by several processes, including fusion, fission, elongation and membrane degradation (Park and Blackstone, 2010).

The rough ER is characterized by being enriched in ribosomes, and is responsible for protein synthesis, folding and post-translational modifications (Bravo et al., 2013; Schwarz and Blower, 2016). On the other hand, smooth ER is free of ribosomes and has tubular structures (Bravo et al., 2013). Moreover, smooth ER is highly dynamic inside the cell due to the ability to fuse, elongate and branch (Bravo et al., 2013). Thus, it is clear that tubular ER and sheet ER have different characteristics, which can explain the distinct roles in different cellular processes. Therefore, the ratio between rough ER and smooth ER can change depending on the cell requirements. An example is pancreatic secretory cells, which are known to synthesize a large amount of secreted proteins, and thus have higher percentage of sheets (Schwarz and Blower, 2016). However, processes associated with lipid synthesis and $\mathrm{Ca}^{2+}$ signaling are related with cells with more tubular network, such as adrenal cells (Schwarz and Blower, 2016).

In the ER, distinct proteins are responsible for the specific shape of ER structures. In the case of tubules or sheets with high curvatures, it is clear that the presence of a very-well known group of proteins belonging to the reticulon family such as the integral membrane protein Reticulon $4 \mathrm{a}$ (Rtn4a) is essential (Shibata et al., 2010; Voeltz et al., 2006). Additionally, in mammals DP1 is another protein that is able to generate tubules by forming oligomers on the smooth ER (Bravo et al., 2013; Shibata et al., 2008). It was shown that overexpression of Rtn4a was able to form more tubules, while depletion of Rtn4a and DP1 contributes to peripheral sheets expansion (Shibata et al., 2008; Voeltz et al., 2006). Some studies have demonstrated the importance of the ER shape, maintenance and proper distribution in several neurological disorders and viral infections, suggesting the relevance of ER dynamics in physiology (Park and Blackstone, 2010; Salinas et al., 2008; Zhao et al., 2001). 


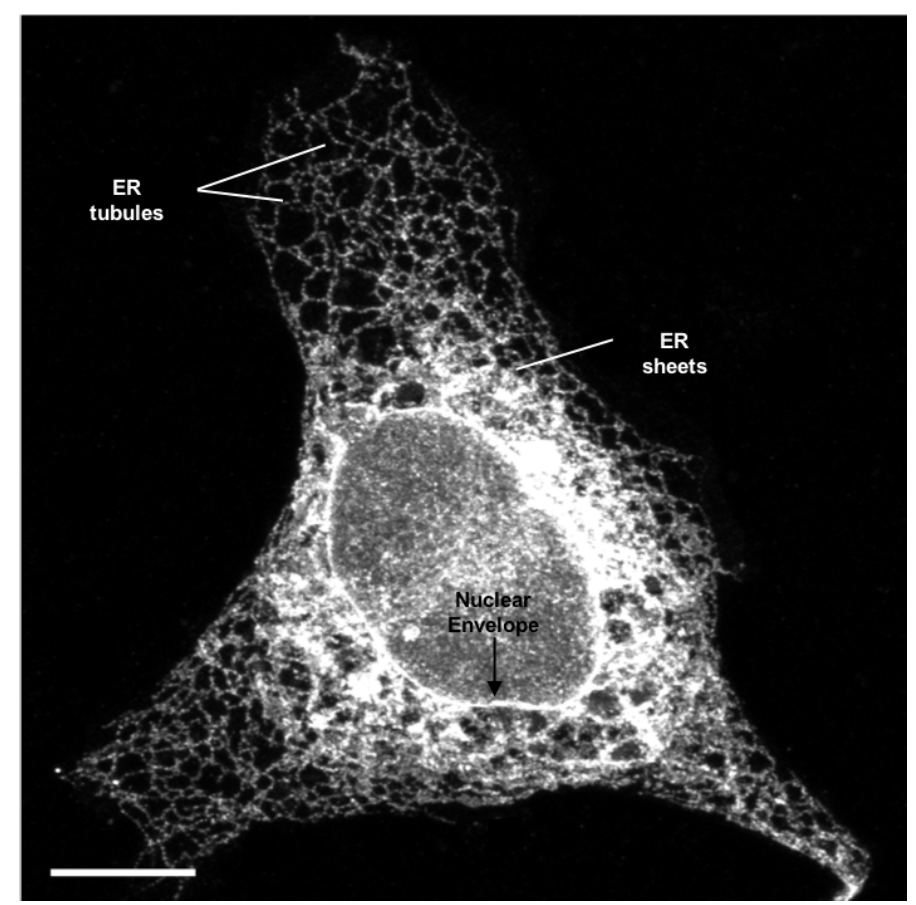

Figure 4 | Endoplasmic reticulum morphology. Confocal image from wild-type mouse embryonic fibroblasts (MEFs) transfected with EGFP-ER marker during 24h. Scale bar $10 \mu \mathrm{m}$.

\subsubsection{Functions of endoplasmic reticulum}

The ER is one of the largest intracellular organelles, and besides being associated with the cellular exo-endocytic pathway, it also performs other fundamental roles in the cell, including lipid and protein synthesis, $\mathrm{Ca}^{2+}$ homeostasis, as well as in the quality control and degradation of proteins (Bravo et al., 2013).

\subsubsection{Protein synthesis and folding}

One of the main functions of the ER is protein synthesis and protein folding (Berridge, 2002). The ribosomes play an essential role in this process, by interacting with mRNAs in the cytosol, and subsequent docking of this complex, ribosome:mRNA, to the ER membrane. This recruitment to the ER membrane occurs via a signal sequence in the newly translated protein, which is recognized and bound by the signal recognition particle (SRP) (Walter, 1981a, 1981b). Then, the translation recommences when the complex is targeted to the ER membrane for docking to the SRP receptor (Gilmore, 1982; Meyer et al., 1982). The proteins are translocated to the ER, and once in ER lumen, 
the ones, which will be secreted undergo proper folding and modifications. The process of folding is supported by chaperones and folding enzymes, determining the destiny of the secretory proteins (Schwarz and Blower, 2016). For the protein secretion process, chaperones are able to release and package them for trafficking to a final destination through the Golgi (van der Zand et al., 2012). However, during this process some failures can occur, and proteins do not achieve native and functional form, and are misfolded and/or aggregated (Hartl and Hayer-Hartl, 2009). In these situations, ER stress response pathways can be activated, including the ER-associated degradation (ERAD) mediated by proteasome. This particular mechanism recognizes and clears these abnormal proteins, preventing them from entering the secretory pathway (Ruggiano et al., 2014). The importance of this pathway is emphasized by reports, which demonstrate that the activation of the ER stress response pathways is associated with some pathologies, such as Alzheimer's disease, diabetes and some cancers (Schwarz and Blower, 2016).

\subsubsection{Calcium homeostasis}

Another crucial role of ER is related with $\mathrm{Ca}^{2+}$ homeostasis in the cell, where this organelle is the major intracellular $\mathrm{Ca}^{2+}$ store (Eisen, 1985; Jaffe, 1983). The ER has several channels and receptors, which are responsible for the release of $\mathrm{Ca}^{2+}$ from the ER to the cytosol, such as ryanodine receptors and inositol 1,4,5-triphosphate $\left(\mathrm{IP}_{3}\right)$ receptors $\left(\mathrm{IP}_{3} \mathrm{R}\right)$ (Clapham, 2005).

The mechanism of $\mathrm{Ca}^{2+}$ release starts in the plasma membrane (PM) with the stimulation of phospholipase C (PLC) by G protein-coupled receptor (GPCR) (Oude Weernink et al., 2007), which in turn cleaves phosphatidylinositol 4,5 biphosphate $\left(\mathrm{PIP}_{2}\right)$ leading to the formation of diacylglycerol (DAG) and $I_{3}$. The generated $I_{3}$ binds to $I P_{3} R$ present in $E R$ membrane, allowing its activation and promoting $\mathrm{Ca}^{2+}$ release to the cytosol (Clapham, 2005).

Moreover, ryanodine receptors (RyRs), predominantly present in excitable cells, are also $\mathrm{Ca}^{2+}$ channels essential for calcium release from the ER to the cytosol. However, their mechanism of release is dependent on intracellular $\mathrm{Ca}^{2+}$ levels, also known as the $\mathrm{Ca}^{2+}$-induced $\mathrm{Ca}^{2+}$ release (CICR) mechanism (Schwarz and Blower, 2016). Additionally, RyRs can also be activated by dihydropyridine receptors, which are voltage-gated channels, and are able to interact with RyRs with subsequent $\mathrm{Ca}^{2+}$ release (Fill and Copello, 2002).

Important to mention is also the entrance of $\mathrm{Ca}^{2+}$ into the ER from the cytosol, which is mainly carried out by a $\mathrm{Ca}^{2+}$ pump called Sarcoendoplasmic reticulum $\mathrm{Ca}^{2+}$-ATPase (SERCA) (Clapham, 2005). In cases of low $\mathrm{Ca}^{2+}$ levels in ER stores, there is activation of a mechanism, which allows $\mathrm{Ca}^{2+}$ entrance into the cell, known as store-operated $\mathrm{Ca}^{2+}$ entry (SOCE) (Clapham, 2005). Therefore, it is 
clear that the ER plays a vital role in intracellular $\mathrm{Ca}^{2+}$ homeostasis, the maintenance of which is essential, due to the distinct roles of $\mathrm{Ca}^{2+}$ in several cellular processes and signaling pathways.

\subsubsection{Lipid synthesis}

The biosynthesis of lipids, in particular glycerophospholipids and sphingolipids, mainly occurs in the ER (Laplante and Sabatini, 2009). Moreover, the enzymes responsible to transform glycerol and fatty acids into triglycerides and diacylglycerol phosphate, the phospholipids precursors, are localized in the ER (Bravo et al., 2013). Furthermore, diacylglycerol phosphate is converted into DAG, which in turn is converted to phosphatidylcholine (PC) and phosphatidylethanolamine (PE) (Bravo et al., 2013). In addition, phosphatidylinositol is also synthesized in the ER, and is essential for signaling and vesicle trafficking (Fagone and Jackowski, 2009). The newly synthesized lipids are then transported to other organelles and cellular membranes via vesicles of the secretory pathway (Van Meer et al., 2008).

\subsubsection{Dynamics of endoplasmic reticulum}

As mentioned above, the smooth ER is very dynamic and is in constant morphological remodeling. In mammalian cells, the important role of microtubules in the ER structure and reorganization is known. Indeed, changes in ER shape were observed after treatment with depolymerizing agents (Bravo et al., 2013). In fact, in 1986 the requirement of microtubules for the maintenance of ER network was already proved (Klopfenstein et al., 1998; Terasaki, 1986). Furthermore, an integral protein, 63-kDa cytoskeleton-linking membrane protein (CLIMP63), which helps the interaction between ER and microtubules was discovered (Klopfenstein et al., 1998).

The ER tubule elongation along microtubules is associated with two different mechanisms: tip attachment complex (TAC) and ER sliding (Bravo et al., 2013). Briefly, the TAC process involves the interaction the ER resident protein, stromal interaction molecule 1 (STIM1) and EB1, a microtubule protein. This interaction promotes ER growth depending on microtubules formation (Bravo et al., 2013; Grigoriev et al., 2008). Regarding ER sliding, two proteins, kinesin 1 and dynein, are responsible to pull ER tubules from the ER membrane together with acetylated microtubules (Friedman et al., 2010; Wozniak et al., 2009). For some cellular mechanisms, such as differentiation or cell division, this interaction between ER and microtubules is crucial for organelle shape remodeling depending on the cell needs (Friedman and Voeltz, 2011). 
ER fusion and branching are also processes occurring in the ER, which are crucial for the formation of a reticular structure (Anderson and Hetzer, 2007). For this process, the presence of proteins called Atlastins, which have GTPase activity, and are able to interact with ER-shaping proteins, promoting ER junction formation is necessary (Barlowe, 2009; Farhan and Hauri, 2009; Hu et al., 2009).

\subsubsection{ER-organelle contacts}

As mentioned above, the ER is one of the biggest organelles in the cell, contributing to the most of membrane contact sites, which have been described in the past years. The ER can have contact sites with several other organelles, including Golgi, mitochondria, PM, lipid droplets and peroxisomes (Wu et al., 2018). The ER-mitochondria is one of the most well studied contact sites, and since it is the main focus of this study, it is discussed in detail in the next section. Another contact site that is well known is the connection between ER and PM. It has been reported that the distance between ER and PM is approximately $30 \mathrm{~nm}$ and this communication has been also associated with several cellular functions, including calcium regulation, PI metabolism and sterol transfer (English and Voeltz, 2013; Schulz and Prinz, 2007; Stefan et al., 2011). In mammals, an example of a protein complex, which has been associated with ER-PM contact sites is Stim1 and Orai1 present in ER and PM, respectively (Liou et al., 2005, 2007). The low $\mathrm{Ca}^{2+}$ in ER can be detected by Stim1, which in turn oligomerizes and interacts with Orai1 on PM, promoting the entrance and restoring of $\mathrm{Ca}^{2+}$ in the ER (Liou et al., 2005, 2007).

Besides, the connection of ER to Golgi apparatus as major components of the secretory pathway, has been also described as physical contacts between these two organelles (Bravo et al., 2013). For example, ceramides which are produced in the ER are transported to the Golgi in order to be converted into sphingomyelin (Hanada et al., 2009; Lev, 2010). Additionally, there is transport of phosphatidylinositol and DAG between the organelles by non-vesicular mechanisms, which seems to require VAP protein and Nir2 protein for phosphatidylinositol transfer (Peretti et al., 2008). Recent studies, also showed an interplay between ER and peroxisomes, which are essentially derived from ER membrane, and also share some metabolic functions such as the synthesis of some phospholipids (Lodhi and Semenkovich, 2014; Wu et al., 2018). Moreover, it is known that acylcoenzyme A binding domain protein 5 (ACBD5) of the peroxisome can interact with VAPs in the ER (Costello et al., 2017; Hua et al., 2017). In fact, an increase of number and surface of ER-peroxisome contact sites was observed after overexpression of either VAPs or ACBD5 (Costello et al., 2017; Hua et al., 2017). Interestingly, the ER can also make connections with lipid droplets with a special 
feature, and there is a membrane continuity between the two organelles (Wu et al., 2018). All the examples mentioned have been reported in mammalian cells. However, it is important to mention that in other organisms, such as in yeast, more contacts and proteins are known, and some of them are conserved, but the machinery to make and keep them is different in eukaryotes (EisenbergBord et al., 2016).

\subsection{Mitochondria-ER contact sites}

In all eukaryotic cells, there is a compartmentalization of specific biological and biochemical functions in organelles. However, nowadays this idea has further evolved, and we can consider that organelles are not isolated and static, and that there is inter-organelle crosstalk. This organelle communication is achieved mostly by physical contact between different organelles or through signaling mechanism from one organelle to the other.

The connections between mitochondria and ER were one of the first membrane contact sites discovered in 1959 through electron microscopy (Copeland, 1959). Afterwards, several aspects about mitochondria-ER contact sites (MERCs) have been discovered.

Nevertheless, at biochemical level the MERCs were only characterized in 1990 by Jean Vance, when she was able to isolate a membrane structure from rat liver, which she defined as the physical contact sites between ER and mitochondria (Vance, 1990). Further, functional characterization was done but only in the late 1990s when Rizzuto and co-workers showed the role of MERCs on $\mathrm{Ca}^{2+}$ transfer between $\mathrm{ER}$ and mitochondria through microdomains of high $\mathrm{Ca}^{2+}$ concentrations upon $\mathrm{ER}$ release (Rizzuto, 1998). Since then, several studies characterizing the function role of MERCs have emerged, and it is now known that MERCs play fundamental roles in several aspects of cellular function. Moreover, in the past several years, there have been a lot of discoveries regarding the MERCs proteome, showing potential proteins associated with MERCs formation and function (De Brito and Scorrano, 2008; Cho et al., 2017; Elbaz-Alon et al., 2015; Filadi et al., 2015; Hung et al., 2017; Simmen et al., 2005; Stoica et al., 2014).

In this chapter several aspects of MERCs are addressed including the structure and composition, as well as the main cellular functions. In addition, mechanistic details of how mitochondria-ER communication can impact some pathologies, such as neurodegenerative diseases, cancer and metabolic disorders are discussed. 


\subsubsection{MERCs: structure and composition in mammals}

The formation of MERCs is facilitated by the presence of proteins in both organelles, which can interact and consequently contribute to the specific properties and functions of MERCs. The distance between ER and mitochondria has been reported to be approximately 10 to $25 \mathrm{~nm}$ (Csordás et al., 2006), which explains how proteins in different organelles can interact, and also the importance of MERCs in several cellular functions. For the past years, the list of proteins associated with MERCs formation or function has been continuously growing. Recently, several experts in the field have reviewed and discussed the real concepts of contact sites and have classified four types of proteins that can be located in the contact sites (Scorrano et al., 2019). The classes can be divided as: structural proteins, functional proteins, sorter/recruitment proteins and regulator proteins (Scorrano et al., 2019). Structural proteins include tethers, which can hold the two organelles together, and spacers that keep both membranes at a defined distance (Scorrano et al., 2019). Regarding the functional proteins, these are proteins that support the function of a specific contact site, and therefore are located and enriched in those areas. Included in this class are proteins, which are able to exchange ions, protein, lipids and metabolites (Scorrano et al., 2019). The contact site proteome and lipidome can be defined by sorting and recruitment proteins. Finally, the last class is defined by proteins that are able to regulate the extent of the contact sites and the function of the active proteins present in this region (Scorrano et al., 2019).

In mammals, one of the protein complexes present in the MERCs is the IP ${ }_{3}$-Grp75-VDAC1, which has been associated with calcium transfer between both organelles (Rapizzi et al., 2002; Szabadkai et al., 2006). The $\mathrm{IP}_{3} \mathrm{R}$ is one of the receptors located at the $E R$, and is responsible for $\mathrm{Ca}^{2+}$ release from this organelle to the cytosol (Foskett et al., 2007). However, IP $P_{3}$ interacts with VDAC1 present in the mitochondria via glucose related regulated protein 75 (GRP75), therefore the calcium released from ER by $I P_{3} R$ is transferred to mitochondria by this interaction in MERCs (Paillusson et al., 2016; Rapizzi et al., 2002; Szabadkai et al., 2006). Nevertheless, it is known that complete loss of $I P_{3} R$ does not affect the MERCs (Csordás et al., 2006; Paillusson et al., 2016) further illustrating the complexity involved in this process.

Also, the homo- and heterotypic interactions between mitochondrial mitofusin $1 / 2$ and ER-located MFN2 have been proposed as a tethering complex (De Brito and Scorrano, 2008). That notwithstanding, there are some controversies regarding the real effect of MFN2 on MERCs, since studies from different laboratories have shown contradictory results (De Brito and Scorrano, 2008; Filadi et al., 2015; Naon et al., 2016). In 2015, Filadi et al. showed in different cell lines that an ablation of MFN2 enhances the MERCs with consistent, efficient $\mathrm{Ca}^{2+}$ transfer from ER to 
mitochondria. In the end, they proposed a model in which MFN2 works as an antagonist of MERCs, avoiding some excessive and toxic proximity between both organelles (Filadi et al., 2015). On the other hand, Scorrano's group has reiterated the role of MFN2 as an agonist of MERCs. In fact, in 2008 they already showed a disruption of MERCs upon silencing of MFN2 in HeLa cells, thus contributing to a reduction on mitochondrial $\mathrm{Ca}^{2+}$ uptake after IP3R stimulation (De Brito and Scorrano, 2008). Additionally, these data were re-evaluated in 2016 by Naon et al., due to the discrepancies in other studies, and the same results were observed after constitutive or acute MFN2 deletion, confirming once again the role of MFN2 as a MERCs tether (Naon et al., 2016).

Moreover, localized in the ER there is a vesicle-associated membrane protein-associated protein $B$ (VAPB), which was shown to bind to the protein tyrosine phosphatase interacting protein 51 (PTPIP51), an outer mitochondrial membrane protein (Stoica et al., 2014; De Vos et al., 2012). The modulation of this protein complex has been demonstrated to induce alterations in the MERCs and consequently affect calcium transfer between the organelles. For example, in a motoneuron-like neuroblastoma cell line, the overexpression or silencing of VAPB and PTPIP51 was shown to strongly increase or decrease the MERCs, respectively (Stoica et al., 2014).

Another complex, formed between the mitochondrial fission protein, Fis1 and BAP31 located at the ER, has been implicated in apoptotic functions of MERCs (Iwasawa et al., 2011). In addition, the complex between phosphofurin acidic cluster sorting protein 2 (PACS2) and Bap31 was associated with MERCs formation, and with a role in controlling cellular homeostasis and apoptosis (Simmen et al., 2005). In 2017, it was shown that a protein located in ER, the PDZ8, which has an orthologous Synaptotagmin-like Mitochondrial lipid-binding protein (SMP) domain, also found in yeast, was required for MERCs formation as well as mediation of $\mathrm{Ca}^{2+}$ transfer between the organelles in mammalian neurons (Hirabayashi et al., 2017). Moreover, an elegant study from Hung et al. discovered a tail-anchored OMM protein, synaptojanin-2 binding protein (SYNJ2BP) using peroxidase-mediated proximity biotinylation approach (Hung et al., 2017). They also showed an increase of MERCs after overexpression of SYNJ2BP, mediated by interaction with ribosomebinding protein 1 (RRBP1), which is present in the ER membrane (Hung et al., 2017). In 2018, the lab from Pietro De Camilli, reported another human protein present in ER, vacuolar protein sortingassociated protein 13A (VPS13A), which can tether to mitochondria, contributing to lipid transport from ER to mitochondria due to the presence of a hydrophobic cavity in the $\mathrm{N}$-terminal portion of VPS13 (Kumar et al., 2018). 


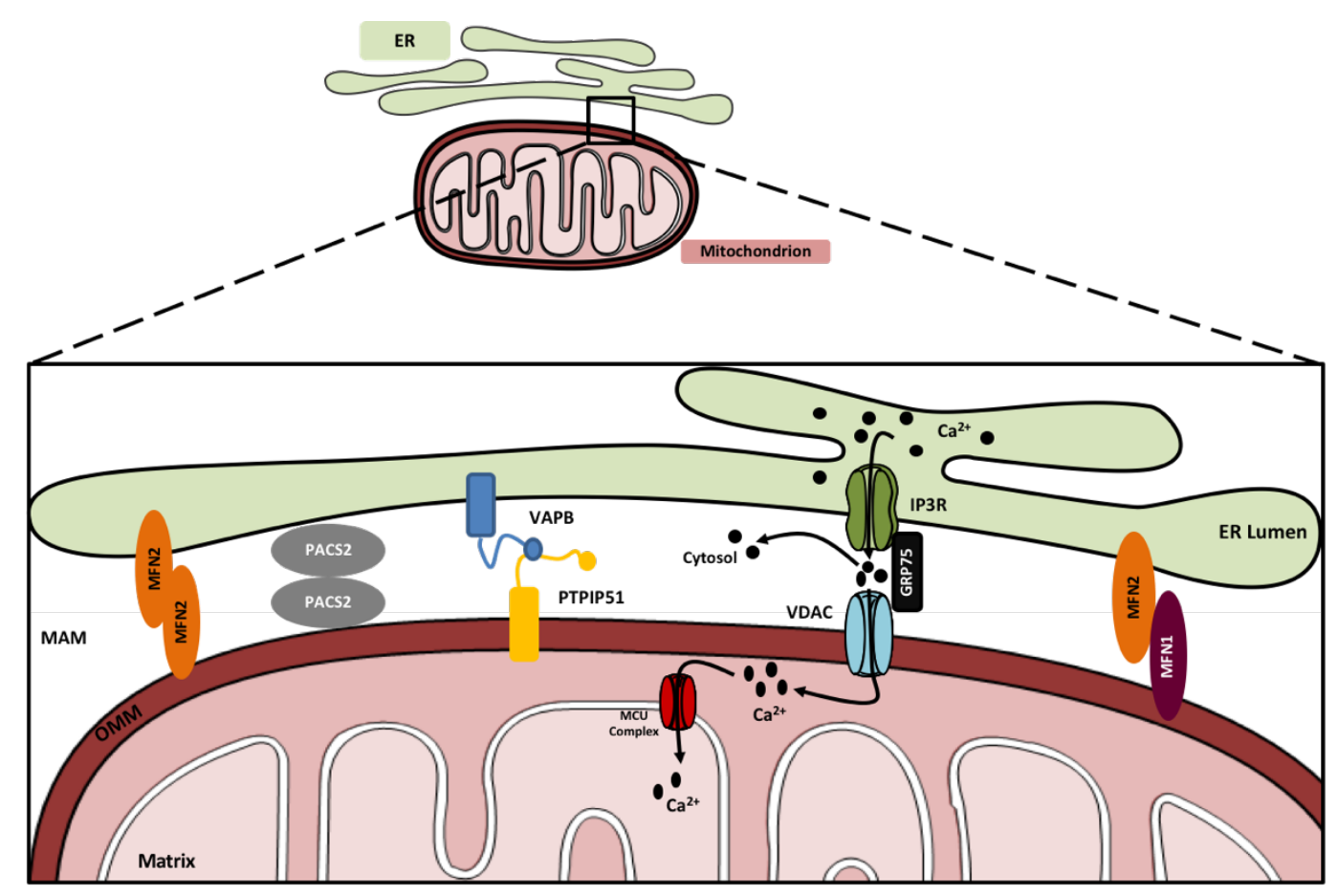

Figure 5 | Illustration of proteins and protein complexes present at ER-mitochondria contact sites (MERCs). MFN2, mitofusin 2; MFN1, mitofusin 1; MAM, mitochondria-associated membranes; VAPB, vesicle-associated membrane protein-associated protein B; PTPIP51, the protein tyrosine phosphatase interacting protein 51; VDAC, voltage-dependent anion channel; $I P_{3} R$, inositol-1,4,5-triphosphate receptor; GRP75, glucose related regulated protein 75 ; $M C U$, mitochondrial $\mathrm{Ca}^{2+}$ uniporters; PACS2, phosphofurin acidic cluster sorting protein 2.

\subsubsection{Control of cellular functions by MERCs in mammals}

\subsubsection{MERCs and lipid transfer}

The biosynthesis of phospholipids is dependent on the crucial functional interplay between ER and mitochondria. In the ER there are enzymes which are required for the synthesis of the majority of phospholipids (Petrungaro and Kornmann, 2019; Vance and Tasseva, 2013). In mammals, the phosphatidylserine (PS) is generated in the ER by the PS synthase (PSS), Which, incidentally, was also the first described component of mitochondria-associated membranes (MAMs)(Stone and Vance, 2000; Vance, 1990; Vance and Tasseva, 2013). In mitochondria, PS is rapidly converted to $P E$ in which a fraction of PE generated is transferred back to the ER, where it is converted to PC or further distributed to other cellular membranes (Figure 6) (Petrungaro and Kornmann, 2019). The conversion to PC requires the hepatic enzyme PE-N-methyltransferase which is enriched in the MERCs (Cui et al., 1993). Taking into consideration this complex interplay, the presence of the close contact sites between the organelles makes sense in order to facilitate lipid transfer through the 
aqueous cytosol. Besides, the fact that mitochondria are not connected to the endomembrane system via vesicular trafficking, meaning that the lipid transfer is just based on non-vesicular transport, makes these contacts more relevant (Petrungaro and Kornmann, 2019).

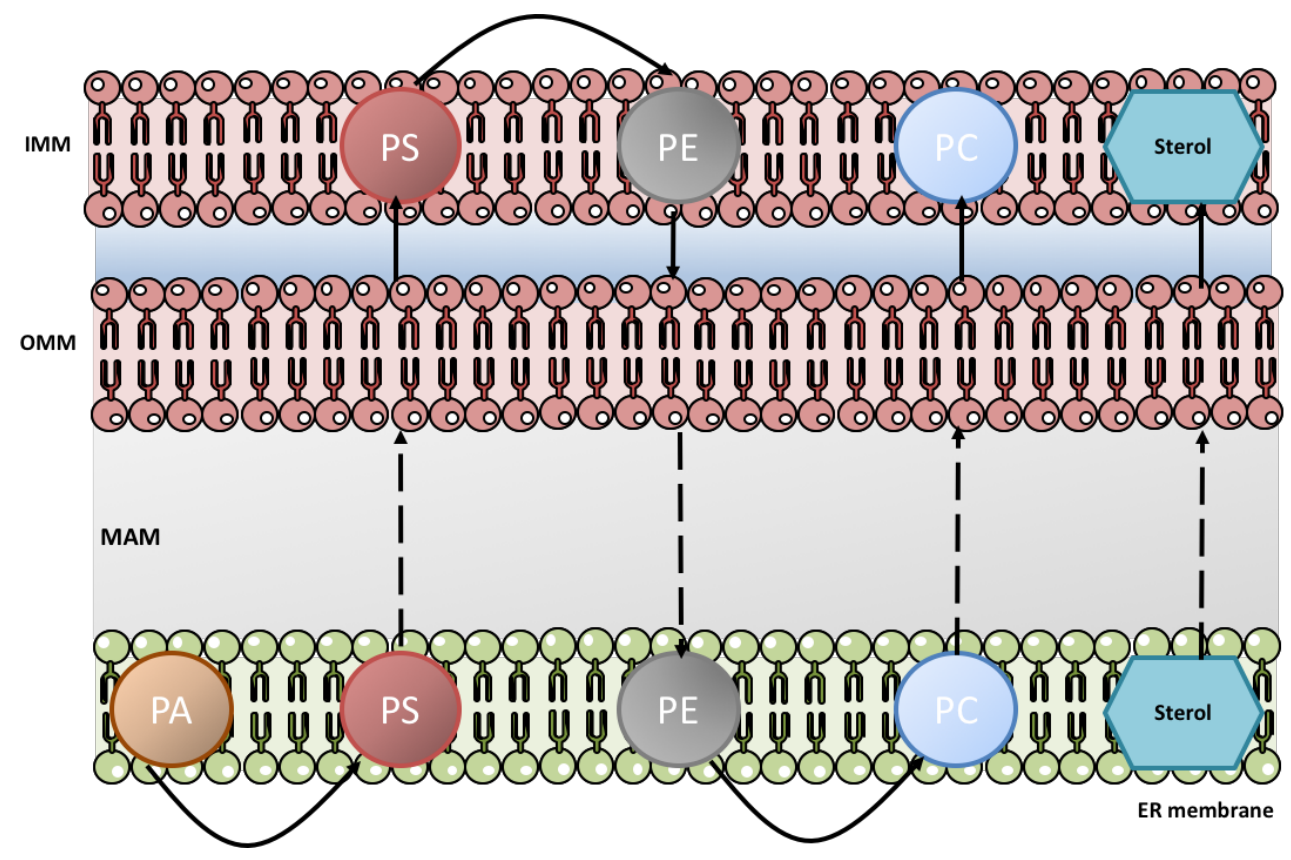

Figure 6 | Mitochondria-ER contact sites (MERCs) function in lipid transfer. In the MERCs, lipids can be transferred between both organelles. In the ER membrane phosphatidic acid (PA) is converted to phosphatidylserine (PS), which in turn is transferred to inner mitochondrial membrane (IMM). In mitochondria, PS is converted to phosphatidylethanolamine (PE) and transferred back to the ER. Part of PE transferred to the ER is converted to phosphatidylcholine (PC), which in turn is transported back to mitochondria. Moreover, sterols can also be transferred between ER and mitochondria at MERCs. IMM, inner mitochondrial membrane; OMM, outer mitochondrial membrane; MAM, mitochondria-associated membranes; PA, phosphatidic acid; PS, phosphatidylserine; PE, phosphatidylethanolamine; PC, phosphatidylcholine.

The MERCs distance $(<30 \mathrm{~nm})$, as well as, the enrichment of lipid-binding proteins and lipid synthesizing enzymes in MAMs, provides a good platform for the non-vesicular lipid transport (Petrungaro and Kornmann, 2019; Vance, 1990). However, this requires some machinery, for example, proteins that are involved in the tethering of both organelles with a role in lipid transport. In the past years, several tethers have been proposed in yeast and as well in mammals (EisenbergBord et al., 2016; Giordano, 2018; Lang et al., 2015; Tamura and Endo, 2017). Moreover, some studies show the importance of lipid transfer proteins (LTPS) in the regulation of lipid composition of the organelle membranes, thus contributing to the non-vesicular lipid transport at MERCs (Helle et al., 2013; Lahiri et al., 2015; Tatsuta et al., 2014). The majority of these studies, based on the 
yeast model, have shown the presence of protein complexes involved in the tethering, as well as in lipid trafficking at MERCs, including ER-mitochondria encounter structure (ERMES) and ERmembrane protein complex (EMC) (Tamura and Endo, 2017). In mammalian cells, several proteins have been described in the past years, however, none has been shown to be involved in lipid trafficking, explaining why is not very well known how lipids can be transferred at MERCs. Nevertheless, as mentioned above, the PDZ8 was recently discovered in MERCs, and it was considered a good candidate for lipid transfer since it has a mammalian SMP domain (Hirabayashi et al., 2017). However, until now, its function on the mitochondrial lipidome has not assessed.

In all eukaryotic membranes, sterols have important roles such as in membrane fluidity, permeability and curvature, as well as in membrane protein function (Petrungaro and Kornmann, 2019). Sterol, once synthesized in the ER, is transported to other organelles, including mitochondria. Although in the mitochondrial membranes the amount of sterols is very low, its importance on mitochondrial morphology is well characterized in yeast (Petrungaro and Kornmann, 2019). Additionally, it is known that in MERCs the cholesterol levels are higher than in the rest of the ER, contributing to the idea that MERCs could favor lipid transfer from ER to mitochondria (Petrungaro and Kornmann, 2019).

As mentioned above, LTPs have been closely associated with the non-vesicular transport between organelles. Indeed, there are four major families of LTPs which are conserved from yeast to mammals, such as oxysterol-binding protein (OSBP)-related proteins (ORP), START, START/VASt, and SMP (Petrungaro and Kornmann, 2019). These proteins have been described as tethers, lipid sensors, or transporter in contact sites. More recently, some proteins belonging to the ORP and Lam/GRAM families, which in fact can be great candidates for mediating lipid transfer, were shown in MERCs (Elbaz-Alon et al., 2015; Petrungaro and Kornmann, 2019). In fact, in 2016 the group of Francesca Giordano showed that knockdowns of ORP5/ORP8 affect mitochondrial morphology and performance, which can be due to the decreased levels of mitochondrial PE (Galmes et al., 2016). Recently, a preprint from the same group showed that ORP5/8 are located in MERCs and mediate non-vesicular transport of PS between ER and mitochondria by physical and functional interaction with MIB/MICOS complexes in mitochondria. However, they also demonstrated a regulation of $\mathrm{Ca}^{2+}$ transfer to mitochondria by ORP5 but not by ORP8 (Rochin et al., 2019). 


\subsubsection{MERCs and calcium regulation}

The major $\mathrm{Ca}^{2+}$ store in mammalian cells is the ER, and the release of $\mathrm{Ca}^{2+}$ is through channels present in the ER membrane, such as $I P_{3} R$ (Foskett et al., 2007). Once $\mathrm{Ca}^{2+}$ is released into the cytosol, it can be taken up into neighboring organelles that require higher levels of $\mathrm{Ca}^{2+}$. In fact, several studies show the crucial role of MERCs in intracellular $\mathrm{Ca}^{2+}$ signaling. Due to the close proximity between both organelles and the formation of $\mathrm{Ca}^{2+}$ microdomains at MERCs, its transfer to mitochondria is direct. Therefore, MERCs have a fundamental role in several aspects of cell metabolism and cell fate determination through cellular $\mathrm{Ca}^{2+}$ regulation (Rizzuto et al., 2009). The entrance of $\mathrm{Ca}^{2+}$ in mitochondria is dependent on $E R \mathrm{Ca}^{2+}$ release. The $\mathrm{IP}_{3} \mathrm{R}$ isoform $3\left(\mathrm{IP}_{3} \mathrm{R} 3\right)$ is present in the MERCs and is involved in $\mathrm{Ca}^{2+}$ transfer from ER to mitochondria through physically interaction with the mitochondrial VDAC1, present in the OMM. This interaction is facilitated by the protein GRP75, which acts as a bridge between IP $_{3}$ R3 and VDAC1 (Figure 7) (Rapizzi et al., 2002; Szabadkai et al., 2006). The IMM is not permeable to $\mathrm{Ca}^{2+}$, however in the IMM there are mitochondrial $\mathrm{Ca}^{2+}$ uniporter (MCU), which allow $\mathrm{Ca}^{2+}$ transfer into the mitochondrial matrix (Baughman et al., 2011; De Stefani et al., 2011). The low $\mathrm{Ca}^{2+}$ affinity of MCU has already been described, therefore higher $\mathrm{Ca}^{2+}$ concentrations at MERCs are required, as well as, an optimal distance between the organelles in order to facilitate an efficient $\mathrm{Ca}^{2+}$ delivery to the mitochondrial matrix (Csordás et al., 2010; Giacomello et al., 2010; De Stefani et al., 2011).

The basal $\mathrm{Ca}^{2+}$ transfer between ER and mitochondria via MERCs is crucial to maintain cellular bioenergetics (Denton, 2009). It is known that $\mathrm{Ca}^{2+}$ can stimulate the activity of some enzymes in the TCA cycle, such as isocitrate and $\alpha$-ketoglutarate dehydrogenases, as well PDH which connects glycolysis with TCA cycle, resulting in more energy production in the cell (Doghman-Bouguerra and Lalli, 2019; Rizzuto et al., 2012). Furthermore, mitochondrial ATP synthase can be activated by $\mathrm{Ca}^{2+}$, thereby contributing to ATP production (Denton, 2009; Territo et al., 2017).

More recently, it was also revealed that $\mathrm{Ca}^{2+}$ transfer at MERCs plays a role in the stimulation of $\mathrm{H}_{2} \mathrm{O}_{2}$ mobilization from mitochondria to MERCs, forming $\mathrm{H}_{2} \mathrm{O}_{2}$ domains in these appositions (Booth et al., 2016). These $\mathrm{H}_{2} \mathrm{O}_{2}$ domains formed can be useful in sustaining cytosolic $\mathrm{Ca}^{2+}$ oscillations by targeting $I P_{3} R$ (Booth et al., 2016).

The efficiency in MERCs calcium exchange is also achieved if there is an efficient $\mathrm{ER} \mathrm{Ca}^{2+}$ storage by SERCA, and its modulation by calnexin, an ER chaperone, also determines the capacity of ER to release $\mathrm{Ca}^{2+}$ (Lynes et al., 2013). Furthermore, there are other proteins which have been associated with mitochondrial $\mathrm{Ca}^{2+}$ uptake, such as Mfn2, PACS-2, Sigma-1 Receptor and Presenilins (BravoSagua et al., 2013). 


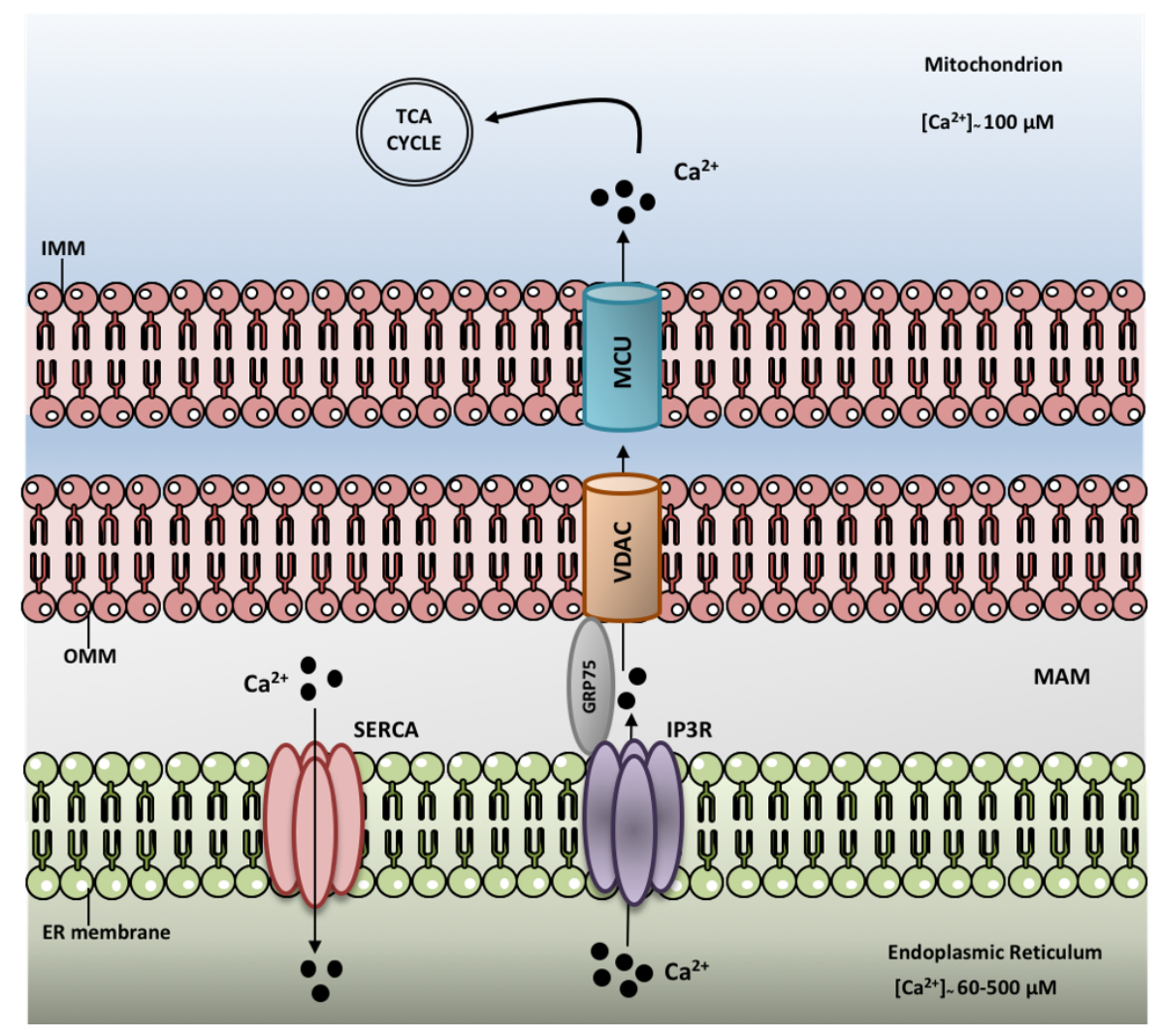

Figure 7 | Calcium $\left(\mathrm{Ca}^{2+}\right)$ transfer occurs at mitochondria-ER contact sites (MERCs). The Ca ${ }^{2+}$ released from the ER through $I P_{3} R$ is directly transferred to mitochondria by voltage dependent channel 1 (VDAC1) located in the outer mitochondrial membrane (OMM). Then, the $\mathrm{Ca}^{2+}$ is transferred into mitochondrial matrix by the mitochondrial $\mathrm{Ca}^{2+}$ uniporter (MCU) present in the inner mitochondria membrane (IMM). Once in mitochondria, this cation can activate some enzymes related to the TCA cycle, improving the bioenergetics of the cell. The ER lumen is the major $\mathrm{Ca}^{2+}$ store in the cell $\left(\left[\mathrm{Ca}^{2+}\right]\right.$ of $\left.60-500 \mu \mathrm{M}\right)$, and in the mitochondria the $\mathrm{Ca}^{2+}$ concentration can reach $100 \mu \mathrm{M}$.

The $\mathrm{Ca}^{2+}$ uptake by mitochondria has to be a very well-regulated process, since an excessive $\mathrm{Ca}^{2+}$ uptake is also associated with mitochondrial impairment and apoptosis induction (Pinton et al., 2008). Mitochondrial $\mathrm{Ca}^{2+}$ overload stimulates apoptosis by activating the mPTP, which in turn leads to the dissipation of the mitochondrial potential, mitochondria swelling and consequently the release of pro-apoptotic factors, such as cytochrome C (CROMPTON, 1999; Naon and Scorrano, 2014). Altogether, it is worthy of note that MERCs can have beneficial effects in the cell. On the other hand, prolonged $\mathrm{Ca}^{2+}$ release, in time or too widespread can be detrimental to the cells (López-Crisosto et al., 2015). 


\subsubsection{Control of cell physiology by MERCs in mammals}

\subsubsection{MERCs and ER stress}

The ER is involved in protein folding, which is a crucial process to maintain protein homeostasis and thus it is enriched in $\mathrm{Ca}^{2+}$ and several chaperones. For this process, ATP is required for chaperone function, as well as for $\mathrm{Ca}^{2+}$ homeostasis. As a result, this is a process with high energy demand. However, in some situations, failures in this process can occur, resulting in an inadequate protein folding due to failure in the ER machinery. This ER state is called ER stress, in which an accumulation of unfolded proteins occurs (Bravo et al., 2013; van Vliet et al., 2014).

Importantly, ER also have an adaptive mechanism called the unfolded protein response (UPR), which is able to restore ER homeostasis by increasing synthesis of chaperones, degradation of misfolded proteins and increasing ER volume (Bravo et al., 2013). It is also important to mention that all these alterations are also accompanied with changes in cellular metabolism. Indeed, it has been shown that in early phases of ER stress there are changes in ER morphology and redistribution, as well as of mitochondrial network towards the perinuclear area of the cell. Additionally, an increase of MERCs in this area of the cell was observed (Bravo et al., 2011). All these alterations are complemented with pieces of evidence showing an increase in mitochondrial $\mathrm{Ca}^{2+}$ uptake, mitochondrial metabolism and ATP production (Bravo et al., 2011). Altogether, this demonstrates that reorganization of both organelles, and the increase of MERCs favors cell survival after ER stress (Bravo et al., 2011). Furthermore, several studies also show the role of other MERCs components on MERCs regulation during ER stress, such as the chaperone sigma-1 receptor (Sig-1R). This chaperone was shown to increase $\mathrm{Ca}^{2+}$ transfer and ATP production (Bravo-Sagua et al., 2013; Shioda et al., 2012). Additionally, MFN2 seems to be involved in ER stress response, since its amount increases after ER stress and on the other hand its deficiency can induce ER stress (Ngoh et al., 2012).

\subsubsection{MERCs in autophagy}

In eukaryotes, autophagy is responsible for the intracellular quality control. It is a well-studied mechanism, which involves the removal and recycling of intracellular content, as well as damaged organelles, and ending in lysosomal degradation (Singh and Cuervo, 2011). However, there is debate about which membrane contributes to autophagosomes formation, the structural hallmark of macroautophagy (Shibutani and Yoshimori, 2014). For some time, it has been known that the ER membranes were contributing to the formation of autophagosomes (Tooze and Yoshimori, 2010). 
Nevertheless, more recently there were pieces of evidence demonstrating that MERCs were involved in the formation of autophagosomes (Hamasaki et al., 2013). Interestingly, a report from Hamasaki and colleagues showed the recruitment of ATG14, a pre-autophagosome marker, to the MERCs under starvation conditions (Hamasaki et al., 2013) further supporting the role of MERCs in autophagosome formation. Consistently, these authors also observed that perturbing the MERCs, by interfering with proteins associated with formation of these contact sites, blocks the localization of ATG14 at MERCs and consequently prevents the proper formation of autophagosomes (Hamasaki et al., 2013). In addition, ATG5, also critical for autophagosome formation, was also found in MERCs during phagophore biogenesis (Hamasaki et al., 2013).

In another study, Rab32, which is required for the formation of autophagic vacuoles (Hirota and Tanaka, 2009), it was shown that Rab32 also has a role in the regulation of MERCs properties (Bui et al., 2010). Furthermore, PACS2 and MFN2 have been associated with an essential role in autophagy regulation since silencing PACS2 and MFN2, which consequently disrupts MERCs formation, was shown to prevent autophagosome formation (Hamasaki et al., 2013).

Recently, it was also discovered that manipulating VAPB and PTPIP51 expression, by silencing or overexpressing, activates or impairs autophagy respectively. These effects are shown to be due to their role in $\mathrm{Ca}^{2+}$ exchange between ER and mitochondria (Gomez-Suaga et al., 2017).

Altogether, these findings support the evidence that MERCs are not only essential for $\mathrm{Ca}^{2+}$ signaling or sensing cellular stress but are also crucial for autophagosome formation and supporting a prosurvival mechanism. Additionally, the recruitment of autophagic machinery at MERCs supports the idea of MERCs function in mitochondrial fission in order to promote mitochondrial clearance (Chu, 2010).

\subsubsection{MERCs and organelle dynamics}

Mitochondria are very dynamic organelles; always undergoing fission and fusion processes, which is crucial in the maintenance of cellular homeostasis (Nunnari and Suomalainen, 2012). The DRP1 is one of the key players in mitochondrial fission (Kraus and Ryan, 2017; Smirnova et al., 2001). In mammalian cells, it is well known that MERCs play a role by marking the constriction sites where the fission process will occur (Friedman et al., 2011). In addition, it is recognized that DRP1 spirals assemble on mitochondria in these contact points (Friedman et al., 2011). Moreover, it is now known that inverted formin-2 in the ER and spire1C present at mitochondria can interact at MERCs. This interaction is responsible for actin filament polymerization and elongation, providing force in the OMM and constriction mitochondria following DRP1 recruitment, and ultimately mitochondrial 
fission occurs (Cohen et al., 2018; Korobova et al., 2013; Manor et al., 2015). In addition, in 2018, a role of actin polymerization at MERCs in stimulating $\mathrm{Ca}^{2+}$ uptake in mitochondria, as well as the constriction of IMM was described (Chakrabarti et al., 2018).

\subsubsection{Organelle pathology: MERCs in disease}

Organelle communication, in particular MERCs, controls several crucial cellular functions such as cell bioenergetics, $\mathrm{Ca}^{2+}$ signaling and lipid transport. Therefore, it is expected that alterations in MERCs could have wide-ranging effects in cell physiology and consequently culminate in some pathologies.

For instance, MERCs have been associated with some neurodegenerative disorders, including Alzheimer disease (AD), Parkinson's disease (PD) and amyotrophic lateral sclerosis with associated frontotemporal dementia (ALS/FTD) (Paillusson et al., 2016). It is known that all the cellular functions regulated by MERCs are impaired in all these neurodegenerative diseases, clearly suggesting the involvement of MERCs in the progression of these diseases.

In sections of brains obtained from human AD patients, an up-regulation of proteins associated with MAMs was observed (Hedskog et al., 2013). In addition, the accumulation of amyloid $\beta$ peptide, characteristic from AD disease, as well as, the overexpression of presenilin 2, enhances the number of MERCs, and contributes to increased $\mathrm{Ca}^{2+}$ transfer between ER and mitochondria (Hedskog et al., 2013; Zampese et al., 2011).

Parkinson's disease is another disease where MERCs dysfunction seems to be present. The familial PD is characterized by mutation in the Parkin and PTEN induced putative kinase 1 (PINK1) genes (Deas et al., 2011; Kitada et al., 1998; Yu et al., 2011). It has been shown that Parkin-mediated mitophagy, and the recruitment of the autophagy machinery occurs in MERCs (Gómez-Suaga et al., 2018; Yang and Yang, 2013). Also, Gelmetti et al. showed that after mitochondrial depolarization, PINK1 and Parkin are re-located to MERCs (Gelmetti et al., 2017; Gómez-Suaga et al., 2018). However, some controversy exists regarding the modulation of these contact sites in a PD model. Some studies have reported that Parkin overexpression increases the MERCs and their functions (Cali et al., 2013). Contrary to the findings, Gautier et al. showed in PARK2 knockout mice and in PD patients that MERCs were enhanced (Gautier et al., 2016).

Furthermore, $\alpha$-synuclein, a protein strongly linked to PD, was shown to be present in MERCs (Guardia-Laguarta et al., 2014). Indeed, a recent study reported that overexpression of wild-type $\alpha$-synuclein increases mitochondrial $\mathrm{Ca}^{2+}$ uptake, and these findings were corroborated by an observed increase of MERCs following $\alpha$-synuclein overexpression(Calì et al., 2012). 
In ALS and FTD, accumulation of tar DNA-binding proteins-43 (TDP-43) is shown to be responsible for the pathology, and mutations in this gene cause also familial ALS and FTD (Paillusson et al., 2016). TDP-43 overexpression reduces MERCs with consequent disruption in $\mathrm{Ca}^{2+}$ exchange (Stoica et al., 2014). This decrease of MERCs has shown to be caused by a disruption of VAPB-PTPIP51 tethers (Stoica et al., 2014). Besides, Sigma 1 receptor, shown to also be responsible for some familial forms of ALS/FTD, has been associated as well with reduction on MERCs following its loss (Bernard-Marissal et al., 2015).

In different cancer types alterations in MERCs were also observed, highlighting the close relationship between processes which are altered during carcinogenesis and MERCs. Notably, several oncogenes and tumor suppressors have been found in MERCs (Sassano et al., 2017). For instance, PACS2 seems to be highly mutated in sporadic colorectal cancer, thus supporting its role as a potential tumor suppressor (Raturi and Simmen, 2013). Also, it seems that the ability of cancer cells to adapt to intracellular stress can be modulated by signaling pathways present at MERCs (Sassano et al., 2017).

Metabolic disorders have been also associated with MERCs disruption (López-Crisosto et al., 2015). For instance, in obese mice an increase of MERCS accompanied with an excessive mitochondrial $\mathrm{Ca}^{2+}$ that resulted in mitochondrial impairment was observed (Arruda et al., 2014). Additionally, PACS2 or IP $\mathrm{R}$ down-regulation in obese mice was reported to ameliorate cellular stress and glucose metabolism (Arruda et al., 2014). Moreover, the role of MERCs on hepatic insulin signaling and nutrient sensing has been characterized (Theurey and Rieusset, 2017). In the liver, overexpression of MERCs proteins enhances insulin signaling, revealing the crucial importance of MAM integrity for insulin signaling (Tubbs et al., 2014). In addition, hypertrophic cardiomyocytes showed insulin signaling disruption due to alterations in mitochondrial $\mathrm{Ca}^{2+}$ levels (Gutiérrez et al., 2014).

\subsubsection{Experimental approaches to investigate MERCs}

In order to elucidate the structural and functional role of MERCs, several approaches have been employed in this field. One of the first methods being used is transmission electron microscopy (TEM), which is a direct and preeminent approach since it offers the required high resolution to quantify the distance between both membranes, the number and the length of MERCs interface (Csordás et al., 2006). Therefore, TEM is considered the "gold standard" method to analyze the contact sites architecture. Besides, it is also possible to couple this technique with tomography, which provides a 3D nature of organelle contacts. However, these techniques are not widely available, and also very time consuming, which makes it not suitable for routine use by researchers 
to evaluate the MERCs. Another strategy that can be used is the proximity ligation assay (PLA) between two contact sites elements present on opposing membranes. This can be useful to analyze alterations in contact sites due to some cellular perturbations. For instance, protein complexes which have been associated with the tethering of ER and mitochondria, such as IP $R$ R-VDAC1 or VAPB-PTPIP51, can be used for this technique (Stoica et al., 2014). However, there are some limitations with PLA, such as the requirement of the localization of PLA partners at the contact sites and requires the proper controls (Scorrano et al., 2019).

A third strategy widely used in organelle research is the epifluorescence and confocal microscopy to evaluate the colocalization of cells expressing fluorescent proteins selectively targeted to the two organelles of interest, or by using markers for the subcellular structures of interest which have been immunostained (Scorrano et al., 2019). This approach has advantages since it allows for live cell imaging, and it is a fast and amenable technique. On the other hand, there are limitations regarding the microscope resolution, which is approximately $250 \mathrm{~nm}$ in $x y$ plane, because the distance between most of the contact sites are far smaller. Second, the chemical fixation for immunofluorescence can introduce some artifacts in the analysis of contact site interactions (Scorrano et al., 2019). Nowadays, more focus is devoted in the field to developing strategies to overcome these limitations, such as fluorescence resonance energy transfer (FRET) and the split fluorescence reporters (Cieri et al., 2018; Scorrano et al., 2019). The FRET-based reporters allow to analyze the proximities between organelles, such as mitochondria and ER, and is based in the transfer of energy between fluorophores: a donor (for example cyan fluorescent protein) and an acceptor (yellow fluorescent protein for example) (Csordás et al., 2010). This technique is useful to investigate the contact sites dynamics and it is very sensitive to the distance between membranes. However, it is important to mention that this is a challenging approach and requires a special microscopy set up (Scorrano et al., 2019). On the other hand, split fluorescence probes, which includes split Venus or green fluorescence protein (GFP), are two non-fluorescent fragments targeted to the partner organelles, and only when these two portions encounter each other that they bind, and a fluorescent signal is emitted (Pinaud and Dahan, 2011). This experimental approach is advantageous since it is possible to discover new contact sites with this technique. It can also be used to perform rescue experiments by using synthetic tethers (Scorrano et al., 2019). In addition to microscopy, the organelle contact sites in particular the MERCs can be explored in the context of biochemical approaches. The MAMs fraction was, for the first time, isolated by the group of J. Vance in the 1990s. This cellular fraction was enriched in ER and OMM membranes (Vance, 1990). This biochemical procedure can be applied for different mammalian tissues and cells, and it is simply based on the isolation of crude mitochondria that is subsequently centrifuged and 
separated from pure mitochondria by gradient centrifugation. With this approach, it is possible to study the composition of MERCs, by analyzing proteins, which are present in the contact sites, as well as to evaluate how the proteins change in response to the cellular environment. In addition, the purified fraction can be subsequently used for mass-spectrometry to reveal other unknown components. Like the other methods, some disadvantages are present, such as contamination from other organelles.

Given the essential role of MERCs in several cellular functions, it is also important to take into account functional studies in order to confirm morphological alterations in MERCs, together with its impact on cellular functions. For this purpose, several functional studies in both cell lines and isolated tissues take advantage of the role of MERCs in $\mathrm{Ca}^{2+}$ transfer and lipid exchange between organelles (De Brito and Scorrano, 2008; Filadi et al., 2015; Galmes et al., 2016; Naon et al., 2016; Stoica et al., 2014; De Vos et al., 2012). The evaluation of $\mathrm{Ca}^{2+}$ exchange is one of the most applied approaches, and it is used in living cells, since it is possible to increase the mitochondrial $\mathrm{Ca}^{2+}$ uptake by stimulating its release from $E R$ in response to some exogenous agents that act on $I P_{3} R$ (for example, histamine). As a result, strategies such as using recombinant targeted protein sensors (Bravo et al., 2011; Rizzuto et al., 1992) or fluorescent chemical indicators (Matsuzaki et al., 2013) have been employed. Regarding the lipid transfer between organelles, it is possible to quantify the conversion of PS-to-PE or the PS content in mitochondria (Vance, 1990). This is possible by using radioisotopes to label phospholipids in order to identify and quantify them by thin layer chromatography (Vance, 1990) or mass spectroscopy (Area-Gomez, 2014; Kainu et al., 2013). 


\subsection{Aims of this study}

Over the last decade, the research based on organelle contact sites and inter-organelle communication has grown, and new insights into its roles in cell physiology have been discovered, namely molecular components and functions of contact sites. In the case of MERCs, they are implicated in several cellular functions (e.g. $\mathrm{Ca}^{2+}$ buffering, lipid trafficking, mitochondrial morphology), and their disruption has been associated with pathological conditions. Despite the fact that MERCs are one of the most studied contact sites, there are still outstanding questions, in particular if and how MERCs respond to cellular signaling, and the basic understanding of their regulation, primarily, their association and dissociation. Additionally, given the molecular complexity of the mitochondria-ER interface, it is plausible to hypothesize its implication in key signaling pathways, including the ones, which are activated following mitochondrial stress. Taken together, the goal of this study was to understand if and how mitochondrial malfunction affects the communication between mitochondria and ER. To this end, the specific aims of this study were to:

- determine the response of MERCs to acute and chronic mitochondrial stress in mammalian cells.

- identify signaling pathways linking mitochondrial malfunction to the formation and stability of MERCs. 


\section{MATERIAL AND METHODS}

\subsection{Material}

\subsubsection{Reagents}

Several chemicals and reagents were used in this study. The respective lists of reagents and their suppliers are provided in the table below.

Table 1 | List of reagents, their suppliers and catalog numbers.

\begin{tabular}{|c|c|c|}
\hline Product & Supplier & $\begin{array}{l}\text { Catalog } \\
\text { number }\end{array}$ \\
\hline 2-Mercapthoethanol & Carl Roth & 4227.3 \\
\hline A769662 & Cayman chemical & 11900 \\
\hline Absolute ethanol & BD Bioscience & 354052 \\
\hline Acrylamide solution $40 \%$ & AppliChem & A0385 \\
\hline Agar 100 Premix kit-hard & Agar Scientific & AGR1140 \\
\hline Agarose & Carl Roth & 3810.3 \\
\hline Ammonium Persulfate (APS) & AMRESCO & 0486 \\
\hline Antimycin & Sigma Aldrich & A8674 \\
\hline Bovine serum albumin (BSA) & Sigma Aldrich & A7906 \\
\hline Bromophenol blue & Carl Roth & T116.1 \\
\hline Cacodylic acid sodium salt trihydrate & Carl Roth & 5169.1 \\
\hline CCCP & Fluka & 857815 \\
\hline Cold fish gelatin & Sigma Aldrich & G7765 \\
\hline Coomassie Brilliant Blue G & Carl Roth & 9598.2 \\
\hline D (+)-Glucose & Carl Roth & 275227941 \\
\hline DAPI & Carl Roth & C6335.1 \\
\hline DMEM High glucose & Gibco & 41965-062 \\
\hline DMEM high glucose + pyruvate & Gibco & 41966-029 \\
\hline DMSO & Sigma Aldrich & D8418 \\
\hline DTT & AppliChem & A2948 \\
\hline $\begin{array}{l}\text { Dulbecco's Modified Eagle's Medium (DMEM) } \\
\text { Base }\end{array}$ & Sigma Aldrich & D5030-1L \\
\hline EDTA & Carl Roth & 8043.2 \\
\hline Ethanol $70 \%$ & VWR & 84858.440 \\
\hline FCCP & Sigma Aldrich & C2920 \\
\hline Fetal Bovine Serum Heat Inactivated & Gibco & $10500-064$ \\
\hline
\end{tabular}


Fugene R6 Transfection Reagent

Promega $\quad$ E2691

\begin{tabular}{|c|c|c|}
\hline GlutaMax-1 (100X) & Gibco & $35050-061$ \\
\hline Glycerol & Carl Roth & 7530.1 \\
\hline Glycine & VWR & 0167 \\
\hline Goat Serum & Life Technologies & $10000 \mathrm{C}$ \\
\hline $\mathrm{H}_{2} \mathrm{O}$ Nuclease-free & VWR & E476 \\
\hline Halt ${ }^{\mathrm{TM}}$ Protease \& Phosphatase single use Inhibitor & Thermo Scientific & 78442 \\
\hline
\end{tabular}

\section{Cocktail}

\begin{tabular}{|c|c|c|}
\hline $\mathrm{HCl}$ & Sigma Aldrich & H1758 \\
\hline HEPES & Carl Roth & HN77.2 \\
\hline $\mathrm{K}_{2} \mathrm{HPO}_{4}$ & Carl Roth & P749.2 \\
\hline $\mathrm{KCl}$ & Volu-Sol & 83608.26 \\
\hline $\mathrm{KH}_{2} \mathrm{PO}_{4}$ & Carl Roth & 3904.1 \\
\hline Methanol & VWR & 20903.368 \\
\hline $\mathrm{MgCl}_{2}$ & VWR & 8.14733 .0100 \\
\hline $\mathrm{MgSO}_{4}-7 \mathrm{H}_{2} \mathrm{O}$ & Sigma Aldrich & 63138 \\
\hline Mowiol 4-88 & AppliChem & A9011 \\
\hline N-dodecylmaltoside & Carl Roth & CN26.2 \\
\hline $\mathrm{NaCl}$ & AppliChem & $\mathrm{A} 1430,0010$ \\
\hline $\mathrm{NaH}_{2} \mathrm{PO}_{4} \cdot \mathrm{H}_{2} \mathrm{O}$ & Carl Roth & K300.1 \\
\hline $\mathrm{NaHCO}_{3}$ & Carl Roth & P029.3 \\
\hline $\mathrm{NaN}_{3}$ & Sigma Aldrich & $52002-1006$ \\
\hline $\mathrm{NH}_{4} \mathrm{Cl}$ & Carl Roth & K298.1 \\
\hline Nonidet ${ }^{\circledR} \mathrm{P}-40$ Substitute & AMRESCO & E109-50ML \\
\hline Oligomycin & Sigma Aldrich & 04876 \\
\hline Osmium tetroxide (OsO4) & Electron Microscopy Sciences & 19134 \\
\hline PageRuler Plus Prestained Protein Ladder & Thermo Scientific & 26619 \\
\hline Paraformaldehyde (PFA) & AppliChem & A3813 \\
\hline PMSF & Sigma & P7626 \\
\hline Polybrene & Sigma & $28728-55-4$ \\
\hline Potassium ferrocyanide & Sigma & P9387 \\
\hline Puromycin 100mg & Fisher Scientific & BP2956-100 \\
\hline SDS & Sigma Aldrich & L4509-500G \\
\hline Skim Milk Powder & Fluka & 70166 \\
\hline TEMED & Sigma Aldrich & T7024 \\
\hline Tris Base & Sigma Aldrich & $\underline{\mathrm{T} 1503}$ \\
\hline Tris- $\mathrm{HCl}$ & Carl Roth & 9090.2 \\
\hline
\end{tabular}




\begin{tabular}{|lll}
\hline Triton $\mathbf{X - 1 0 0}$ & AMRESCO & 0694 \\
\hline Trypan Blue $\mathbf{0 . 4 \%}$ & Life Technologies & T10282 \\
\hline Tween $\mathbf{2 0}$ & AMRESCO & $\mathbf{0 7 7 7 - 1 L}$ \\
\hline Uranyl acetate (UA) & Agar Scientific & AGR1260A \\
\hline Uridine & Sigma Aldrich & U3003
\end{tabular}

\subsubsection{Kits and disposables}

The kits and disposables used throughout this thesis, with their corresponding suppliers and catalog numbers, can be found in the table below (Table 2).

Table 2 | List of kits and disposables used in the study.

\begin{tabular}{|c|c|c|}
\hline Product & Supplier & Catalog number \\
\hline Amersham Hybond PO45 PVDF & GE Healthcare & 10600023 \\
\hline Centrifuge tubes $15 \mathrm{~mL}$ Corning CentriStar & Corning & 430791 \\
\hline Centrifuge tubes $50 \mathrm{~mL}$ Corning CentriStar & Corning & 430829 \\
\hline CRYSTAL RNA Mini Kit & New England Biolabs & $31-010-404$ \\
\hline Cuvettes PMMA & VWR-International & $634-0678$ \\
\hline FACS Tubes Polystyrene Round-Bottom 5 mL & Corning & 352052 \\
\hline High Performance chemiluminiscence film & GE Healthcare & 28906837 \\
\hline Lenti $X$ concentrator & Clontech & 631231 \\
\hline Luminata $^{\mathrm{TM}}$ Classico Western HRP Substrate & Millipore & WBLUC0500 \\
\hline Luna $^{\circledR}$ Universal qPCR Master Mix & New England BioLabs & M3003 \\
\hline Medical X-Ray Film & Foma & ----- \\
\hline Microscope slides & Carl Roth & 2111 \\
\hline MitoSOX ${ }^{\mathrm{TM}}$ Red & Life Technologies & M36008 \\
\hline PBS tablets & Sigma Aldrich & Р44177-100TAB \\
\hline Penicillin/Streptomycin & Gibco & $15140-062$ \\
\hline Pierce BCA Protein Assay Kit & Thermo Scientific & $23225 i$ \\
\hline Protein Assay Dye Reagent Concentrate & Bio-Rad & $500-0006$ \\
\hline Serological Pipette $10 \mathrm{~mL}$ & StarLab & E4860-1011 \\
\hline Serological Pipette 25 mL & StarLab & E4860-2511 \\
\hline Serological Pipette $5 \mathrm{~mL}$ & StarLab & E4860-0511 \\
\hline Sodium Pyruvate & Sigma & S8636 \\
\hline Test Tube Soda Glass & VWR-International & $212-003$ \\
\hline
\end{tabular}




\begin{tabular}{|c|c|c|}
\hline Tips $10 \mu \mathrm{L}$ TipOne & StarLab & S111-3210 \\
\hline Tips $1000 \mu \mathrm{L}$ TipOne & StarLab & S1111-6001 \\
\hline Tips $20 \mu \mathrm{L}$ TipOne & StarLab & S120-1810 \\
\hline Tips $200 \mu$ L TipOne & StarLab & S1120-8800 \\
\hline Triple ${ }^{t}$ Express (1X) & Gibco & $12605-010$ \\
\hline Trypan Blue $0.4 \%$ & Life Technologies & T10282 \\
\hline Tubes $0.5 \mathrm{~mL}$ molecular probes & Life Technologies & Q33856 \\
\hline Tubes $1.5 \mathrm{~mL}$ & StarLab & E1415-1500 \\
\hline Tubes $2 \mathrm{~mL}$ & StarLab & S1620-2700 \\
\hline Western Blot Paper & TH-Geyer & $4-01-60-0041$ \\
\hline $\mathrm{XF}^{\mathrm{e}} 96$ extracellular flux assay kit Seahorse & BD Bioscience & $102416-100$ \\
\hline
\end{tabular}

\subsubsection{Buffers and solutions}

All buffers and solutions used in this study are listed below in Table 3. Unless otherwise stated, all buffers and solutions were prepared in ${ }_{\mathrm{dd}} \mathrm{H}_{2} \mathrm{O}$ and concentrated buffers were further diluted to $1 \mathrm{X}$ in ${ }_{d d} \mathrm{H}_{2} \mathrm{O}$ also.

Table 3 | List of buffers and solutions.

\begin{tabular}{|c|c|}
\hline Buffer/solution & Composition \\
\hline $\begin{array}{l}\text { Blocking buffer } \\
\text { (Immunoblotting) }\end{array}$ & $5 \%(w / v)$ Skimmed milk or BSA in TBST \\
\hline $\begin{array}{l}\text { Blocking buffer } \\
\text { (Immunocytochemistry) }\end{array}$ & $\begin{array}{l}0.1 \% \text { Cold fish gelatin, } 0.1 \% \text { Triton } \mathrm{X}-100,3 \% \mathrm{BSA}, 1 \% \text { Goat serum and } \\
0.05 \% \text { Tween-20 in PBS }\end{array}$ \\
\hline Cacodylate buffer & $0.2 \mathrm{M}$ of Cacodylic acid sodium salt trihydrate in ddH2O, $\mathrm{pH} 7.4$ \\
\hline Ferrocyanide solution & $1.5 \%(\mathrm{w} / \mathrm{v})$ of ferrocyanide in $\mathrm{ddH} 2 \mathrm{O}$ \\
\hline $\begin{array}{l}\text { Freezing medium } \\
\text { (Cell culture) }\end{array}$ & $10 \%(v / v)$ DMSO, $20 \%$ (v/v) FBS in DMEM high glucose \\
\hline Mowiol & 6.4mM Mowiol 4-88, 5.4M Glycerol, 0.1M Tris pH 8.5 \\
\hline MS homogenization buffer & $\begin{array}{l}210 \mathrm{Mm} \text { mannitol, } 70 \mathrm{mM} \text { sucrose, } 5 \mathrm{mM} \text { Tris- } \mathrm{HCl}, 1 \mathrm{mM} \text { EDTA, } \mathrm{pH} \\
7.5\end{array}$ \\
\hline NaN3 & $10 \% \mathrm{NaN3}$ \\
\hline Osmium tetroxide (OsO4) & $4 \%(\mathrm{w} / \mathrm{v})$ of $\mathrm{OsO} 4$ in $0.1 \mathrm{M}$ cacodylate buffer \\
\hline PBS & 5 PBS tablets in $1 \mathrm{~L} \mathrm{ddH2O}$ \\
\hline
\end{tabular}




\begin{tabular}{|c|c|}
\hline Resolving gel $12 \%$ & $\begin{array}{l}0.375 \mathrm{M} \text { Tris, } \mathrm{pH} 8.8,0.1 \% \mathrm{SDS}, 12 \% \text { Polyacrylamide, } 0.1 \% \text { APS, } 0.04 \% \\
\text { TEMED }\end{array}$ \\
\hline Ringer's buffer & $\begin{array}{l}55 \mathrm{mM} \mathrm{NaCl}, 4.5 \mathrm{mM} \mathrm{KCl}, 10 \mathrm{mM} \text { glucose, } 5 \mathrm{mM} \text { HEPES, } 2.75 \mathrm{mM} \\
\mathrm{MgCl} 2,0.5 \mathrm{mM} \mathrm{CaCl} 2, \mathrm{pH} 7.437^{\circ} \mathrm{C}\end{array}$ \\
\hline RSB hypo buffer & 10 mM NaCl, 1.5 mM MgCl2, 10 mM Tris-HCl, pH 7.5 \\
\hline Running buffer $1 \mathrm{x}$ & $5 \mathrm{mM}$ Tris, $192 \mathrm{mM}$ glycine, $0.1 \%$ SDS \\
\hline SDS loading buffer & $\begin{array}{l}\text { 62.5mM Tris, pH 6.8, } 50 \%(\mathrm{v} / \mathrm{v}) \text { Glycerol, } 12 \%(\mathrm{w} / \mathrm{v}) \mathrm{SDS}, 0.06 \%(\mathrm{w} / \mathrm{v}) \\
\text { Bromophenol blue. Add 5\% 2-Mercaptoetanol freshly prior to use }\end{array}$ \\
\hline Stacking gel $4 \%$ & $\begin{array}{l}\text { 62.5mM Tris pH 6.8, 0.1\% SDS, } 4 \% \text { Polyacrylamide, } 0.1 \% \text { APS, } 0.05 \% \\
\text { APS \% TEMED }\end{array}$ \\
\hline Sucrose density gradient & 1.0 M sucrose or $1.5 \mathrm{M}$ sucrose, $10 \mathrm{mM}$ Tris- $\mathrm{HCl}, 1 \mathrm{mM}$ EDTA, $\mathrm{pH} 7.5$ \\
\hline TBST 10X & 200mM Tris, 1.5M NaCl, 1\% Tween 20, pH 7.4 \\
\hline TE buffer & 10mM Tris- $\mathrm{HCl}, 0.2 \mathrm{mM}$ EDTA pH 7.5 \\
\hline Transfer buffer 10X & 250mM Tris, 1.92M Glycine. $1 X$ dilution includes 20\% (v/v) Methanol \\
\hline Uranyl Acetate (UA) & $4 \%(w / v)$ of $U A$ in $d d H 2 O$ \\
\hline Whole cell extraction buffer & $\begin{array}{l}1.5 \% \mathrm{~N} \text {-dodecylmaltoside in PBS supplemented with fresh } 1 \mathrm{X} \\
\text { protease/phosphatase inhibitor }\end{array}$ \\
\hline
\end{tabular}

\subsubsection{Antibodies}

Different primary and secondary antibodies were used for immunoblotting (IB) and immunocytochemistry (ICC) experiments in this thesis. These antibodies are listed below.

Table 4 | Primary antibodies for immunoblotting and immunocytochemistry.

\begin{tabular}{|c|c|c|c|c|}
\hline Antibody & Supplier & Catalog number & Application & Dilution \\
\hline ACC & Cell signaling & 3676 & IB & 1:1000 \\
\hline ACC pSer ${ }^{79}$ & Cell signaling & 3661 & IB & 1:1000 \\
\hline AMPK $\alpha 1$ & Cell Signaling & 2795 & IB & 1:1000 \\
\hline AMPK $\alpha 1$ & Novus Biologicals & NBP2-22127 & IB & 1:1000 \\
\hline DRP1 & BD Biosciences & BD-611113 & IB/ICC & 1:1000/1:50 \\
\hline GAPDH & Sigma Aldrich & G9545 & IB & 1:10000 \\
\hline HPRT & Abcam & ab10479 & IB & $1: 4000$ \\
\hline MFF & Proteintech & 17090-1-AP & IB & 1:1000 \\
\hline MFN2 & abcam & $a b 124773$ & IB & 1:1000 \\
\hline S6 & Cell signaling & 2217 & IB & 1:2000 \\
\hline S6 pSer ${ }^{235 / 236}$ & Cell signaling & $4858 S$ & IB & $1: 2000$ \\
\hline
\end{tabular}




$\begin{array}{lllll}\text { TOM20 } & \text { Proteintech } & \text { 11802-1-AP } & \text { IB/ICC } & 1: 1000 / 1: 200 \\ \text { UQCRC1 } & \text { Abcam } & \text { ab110252 } & \text { IB } & 1: 2000 \\ \text { VAPB } & \text { biomol } & \text { A302-894A-M } & \text { IB } & 1: 1000 \\ \text { VDAC1 } & \text { abcam } & \text { ab14734 } & \text { IB } & 1: 1000\end{array}$

Table 5 | Secondary antibodies for immunoblotting and immunocytochemistry.

\begin{tabular}{llll} 
Antibody & Supplier & Code & Applications \\
\hline Goat anti-mouse Alexa 568 & Life Technologies & A-11031 & ICC \\
\hline Goat anti-mouse IgG & Dianova & $115-035-146$ & IB \\
\hline Goat anti-rabbit Alexa 488 & Life Technologies & A-11008 & ICC \\
\hline Goat anti-rabbit IgG & Dianova & $115-035-144$ & IB
\end{tabular}

\subsubsection{Cell culture materials}

The following materials and devices were used specifically for cell culture experiments carried out in this study.

Table 6 | Cell culture materials and devices.

\begin{tabular}{|c|c|c|}
\hline Product & Supplier & Catalog number \\
\hline $100 \times 20 \mathrm{~mm}$ TC dish & Starlab & CC7682-3394 \\
\hline 12-well TC plate & Starlab & CC7682-7512 \\
\hline $150 \times 20 \mathrm{~mm}$ TC dish & Starlab & CC7682-3617 \\
\hline 6-well TC plate & Starlab & CC7682-7506 \\
\hline $60 \times 20 \mathrm{~mm}$ TC dish & Starlab & CC7682-3354 \\
\hline 96-well TC plate & Starlab & CC7682-7596 \\
\hline Cell scraper & StarLab & CC7600-0202 \\
\hline Countess cell counting chamber slides & Invitrogen & C10283 \\
\hline Coverslips & Marienfeld & 017580 \\
\hline Cryogenic vial 2 mL & Fisher Brand & 1050026 \\
\hline Filter syringe $0.22 \varnothing$ Rotilabo CME & Carl Roth & SE2M35I07 \\
\hline Syringe Inject & Becton. Dickinson and Company & 4606205 \\
\hline
\end{tabular}




\subsubsection{Plasmids}

The transfections in this study were performed with pcDNA and shRNA listed below (Table 9).

Table 7 | Plasmids and shRNA used in this study.

\begin{tabular}{|c|c|c|}
\hline Plasmid & Supplier & Catalog number \\
\hline 4mtD3cpv & \multicolumn{2}{|c|}{ Kind gift from Prof. Dr. Ivan Bogeski } \\
\hline EGFP-TMsac1 & \multicolumn{2}{|c|}{ Kind gift from Dr. Ira Milosevic } \\
\hline Mito-mcherry & \multicolumn{2}{|c|}{ Kind gift from Prof. Dr. Stefan Jakobs } \\
\hline $\begin{array}{l}\text { pLenti.PGK.blast-Flag-MFF- } \\
\text { S155,172A }\end{array}$ & Addgene & 74380 \\
\hline enti.PGK.blast-Flag-MFF-S155,172D & Addgene & 74442 \\
\hline pLenti.PGK.blast-Flag-MFF-WT & Addgene & 74379 \\
\hline pLKO.1-blast-scrambled & Addgene & 26701 \\
\hline PLP1, PLP2, PLP-VSVG & \multicolumn{2}{|c|}{ Kind gift from Prof. Dr. Katschinski } \\
\hline Scrambled negative control & Integrated DNA Technologies & 51-01-19-09 \\
\hline ShRNA-UQCRC1 & GE Dharmacon & TRCN0000046483 \\
\hline ShRNA-UQCRC1 & GE Dharmacon & TRCN0000046484 \\
\hline ShRNA-UQCRC1 & GE Dharmacon & TRCN0000046485 \\
\hline shRNA-UQCRC1 & GE Dharmacon & TRCN0000046486 \\
\hline ShRNA-UQCRC1 & GE Dharmacon & TRCN0000046487 \\
\hline
\end{tabular}

\subsubsection{Equipment}

All the equipment used in this study is listed in Table 10 below.

Table 8 | List of equipment.

\begin{tabular}{ll} 
Equipment & Manufacturer \\
\hline Ace Block Digital Dry Bath & Labnet \\
\hline Amaxa 4D-Nucleofector & Lonza \\
\hline Bio-Rad Power Pack HC Mini-Protean Tetra System & Bio-Rad \\
CAWOMAT 2000 & CAWO \\
Cell Observer High Speed microscope & Zeiss \\
\hline Centrifuge 5415R & Eppendorf \\
\hline Centrifuge 5810R & Eppendorf
\end{tabular}


Centrifuge Allegra X-15R

\begin{tabular}{ll}
\hline Countess C10281 & Invitrogen \\
\hline FACS Canto ${ }^{T M}$ II & BD Biosciences \\
\hline Gene Quant 1300 & GE Healthcare \\
\hline Hood Herasafe & Thermo Scientific \\
\hline Incubator Heracell 150i & Thermo Scientific \\
\hline JEM 1011 transmission electron microscope & JEOL \\
\hline Microscope Zeiss Axio Vert A1 & Zeiss \\
\hline Nanodrop $2000 C$ & Peqlab \\
\hline Potter S homogenizer & Sartorius \\
\hline Quant Studio 6 Flex & Life Technologies \\
\hline Scanner Epson Perfection V850 Pro & Epson \\
\hline SE600 Ruby system & GE Healthcare \\
\hline Seahorse XF96 extracellular Flux Analyzer & Agilent \\
\hline SYNERGYM1 microplate reader & BioTek \\
\hline Vortex RS-VA10 & Phoenix Instrument \\
\hline Zeiss LSM 800 Airyscan & Zeiss \\
\hline
\end{tabular}

\subsubsection{Software}

Several software were used to analyze most of the data obtained from experiments carried out in this study. The table below lists these software packages and their manufacturers

Table 9 | List of software.

\begin{tabular}{llll} 
Software & Manufacturer & City/State & Country \\
\hline Adobe Illustrator CS6 & Adobe Systems Inc. & San Jose, CA & USA \\
\hline FACS DIVA $^{\text {TM }}$ software & BD Biosciences & Heidelberg & Germany \\
\hline FlowJo & FlowJo LLC & Ashland, OR & USA \\
\hline GraphPad Prism 6 \& 7 & GraphPad Software Inc. & La Jolla, CA & USA \\
\hline ImageJ & NIH & Bethesda, MD & USA \\
\hline Imaris & Bitplane & Zurich & Switzerland \\
\hline Mendeley & Mendeley Ltd. & London & UK \\
Microsoft Office & Microsoft Corporation & Redmond, WA & USA \\
Velocity & Perkin Elmer & Waltham, MA & USA
\end{tabular}




\subsection{Methods}

\subsubsection{Cell Culture}

\subsubsection{Cell lines and culture growth conditions}

In the present work, HeLa cells and mouse embryonic fibroblasts (MEFs) were used.

The HeLa cell line was purchased from ATCC (LGC Standards, Middlesex, UK). AMPK MEFs, AMPK $\alpha 1^{-/} \alpha 2^{-/-}$and the corresponding wild-type, were a kind gift of Dr. Benoit Viollet (Institut Cochin, Paris, France). MFF"- MEFs and the corresponding wild-type, were a kind gift of Dr. David Chan (Caltech, California, USA). All the cell lines used were stored frozen in liquid nitrogen vapor phase and expanded according to manufacturer's instructions.

HeLa RC-kd and scrambled control cells were grown in sterile Dulbecco's Modified Eagle Medium high glucose medium (DMEM) with $1 \mathrm{mM}$ pyruvate supplemented with $10 \%$ fetal bovine serum (FBS), $1 \%$ Penicillin/Streptomycin (P/S) and $200 \mu \mathrm{M}$ uridine at $37^{\circ} \mathrm{C}$ and $5 \% \mathrm{CO}_{2}$. All MEFs used were cultured in sterile DMEM supplemented with $10 \% \mathrm{FBS}$ and $1 \% \mathrm{P} / \mathrm{S}$ at $37^{\circ} \mathrm{C}$ and $5 \% \mathrm{CO}_{2}$. Cells were seeded at least 24 hours before experiments and harvested at $70-80 \%$ confluence for all experiments. For routine subculturing, cells were first rinsed with sterile $1 x$ Phosphate Buffered Saline (1x PBS; 0.137 M NaCl, $2.7 \mathrm{mM} \mathrm{KCl}, 1.4 \mathrm{mM} \mathrm{KH2PO4,0.01} \mathrm{M} \mathrm{Na2HPO4)} \mathrm{and} \mathrm{then}$ incubated with 1 volume of trypsin-EDTA for $5 \mathrm{~min}$ at $37^{\circ} \mathrm{C}$. Trypsin activity was inhibited by the addition of 1 volume of complete growth medium and the final volume centrifuged at $1000 \mathrm{xg}$ for $3 \mathrm{~min}$ at room temperature. An appropriate aliquot of the cell suspension was added to new culture flasks. This procedure was repeated when cultures reached $80-90 \%$ confluence.

\subsubsection{Generation of stable knock down and overexpression cell lines}

Lentiviral stable knockdown generation was done by growing HEK293T packaging cells in DMEM high glucose (Gibco) supplemented with $10 \%$ FBS and after $24 \mathrm{~h}$ cells were transfected with viral components (an optimized mix of packaging plasmids PLP1, PLP2 and PLP/VSVG) and shRNA against target genes (or scrambled control) using Lipofectamine 2000, grown and concentrated using Lenti-X Concentrator (Clontech) according to the manufacturer's instructions. The shRNA constructs were purchased from Open Biosystems (Dharmacon). HeLa cells were then seeded at 12000 cells/cm2 and grown overnight to $70-80 \%$ confluence. These cells were transduced with the lentiviral particles using Polybrene $(8 \mu \mathrm{g} / \mathrm{ml})$ and puromycin was used as selection agent. For stable knockdown, efficiency was confirmed by Western blot. The best knockdown was selected for subsequent experiments. For stable MFF overexpression, lentiviral suspensions 
were obtained as described before using packaging and envelope viral vectors with MFF constructs (purchased from Addgene, Cambridge, USA). AMPK DKO MEFs were then seeded at $1 \times 10^{5}$ cells and grown overnight to $70-80 \%$ confluence. These cells were transduced with the lentiviral particles using Polybrene $(8 \mu \mathrm{g} / \mathrm{ml})$ and puromycin resistant cells were selected.

\subsubsection{Transfection of HeLa RC-kd and mouse embryonic fibroblasts}

HeLa RC-kd cells were seeded $24 \mathrm{~h}$ before transfection in 12-well-plates with coverslips coated with poly-lysine for microscopy. Afterwards, cells were transfected using FuGENE HD Transfection Reagent ( $1 \mu \mathrm{g}$ of DNA: $3 \mu \mathrm{L}$ of FuGENE). The transfecting mix was prepared by adding $3 \mu \mathrm{L}$ of FuGENE directly into $50 \mu \mathrm{L}$ of Opti-MEM medium, mixed and incubated for 5 minutes at room temperature. In the meanwhile, in another microcentrifuge tube, $50 \mu \mathrm{L}$ of OptiMEM medium and $1 \mu \mathrm{g}$ of respective DNA was prepared and mixed. Finally, DNA dilution was added to the FuGENE dilution, mixed and incubated for 20 minutes at room temperature. Following incubation, the transfection mix was added to the wells dropwise and gently mixed. 6 hours post-transfection, the medium was changed with fresh medium and cells were kept for 24 hours at $37^{\circ} \mathrm{C}$ and $5 \% \mathrm{CO}_{2}$.

Mouse embryonic fibroblasts were transfected with respective plasmids using electroporation, following manufacturer's instructions. Briefly, after collecting cells as described before, the cell pellet was resuspended in warm growth medium and $10 \mu \mathrm{L}$ of the suspension was applied to an improved Neubauer cell counting chamber. The cell density was determined by the formula: $N \times 10^{4}$ where $N$ is the average cell number in the four corner squares. Following cell density determinations, $5 \times 10^{5}$ cells were pelleted and the excess medium aspirated. $20 \mu \mathrm{L}$ of Amaxa transfection working reagent (made of $263 \mu \mathrm{L}$ reagent $A$ and $62.5 \mu \mathrm{L}$ reagent $B$ ) was added to the cell pellet. Plasmids $(1 \mu \mathrm{g})$ were added and the cell pellet was resuspended by gently pipetting up and down. The cell suspension was transferred to labeled electroporation small cuvettes and electroporated with the appropriate program for dermal MEFs using the Amaxa 4D Nucleofector. The electroporated cells were then incubated at room temperature for 10 minutes, resuspended in warm growth medium and seeded $\left(3 \times 10^{4}\right.$ cells/well) into appropriate 12-well plates for microscopy. The transfected cells were cultured in normal growth conditions at $37^{\circ} \mathrm{C}$ and $5 \% \mathrm{CO}_{2}$ and fixed after 24 hours. 


\subsubsection{Material harvesting}

\subsubsection{Cells harvesting for total protein}

In order to prepare the whole cell lysates, adherent cells were harvested by aspirating the growth medium and washed once in PBS $1 x$. Then, cells were scraped on ice with ice cold PBS $1 \mathrm{x}$ and the cell suspension was collected and pelleted at $2500 \mathrm{rpm}, 4^{\circ} \mathrm{C}$ for 5 minutes. The cell pellet was used for the required technique or frozen in liquid nitrogen and stored at $-80^{\circ} \mathrm{C}$ for later use. For specific techniques, such as flow cytometry, cryopreservation and transfection of MEFs (described later), the whole cell extract was prepared differentially using trypsinization. For this purpose, the growth medium was aspirated, and adherent cells were washed with warm PBS $1 x$ and. Then, TrypLE Express enzyme was added for cell detaching, and cells were incubated during 5 minutes at $37^{\circ} \mathrm{C}$. The trypsin activity was inhibited by adding twice the volume of growth medium and subsequently, the cell suspension was collected and subjected to centrifugation at $1,500 \mathrm{rpm}, 4{ }^{\circ} \mathrm{C}$ for 5 minutes. the cell pellet used for further experiments and the supernatant was discarded.

\subsubsection{Subcellular fractionation}

The crude mitochondria and pure mitochondria were prepared as described previously with some modifications (Clayton and Shadel, 2015; Clayton et al., 2014). The preparation of further subcellular fractions (microsomes and cytosol) was adapted from Wieckowski et al. (Wieckowski et al., 2009). Briefly, AMPK MEFs grown on 15-cm dishes were resuspended in RSB hypo buffer $(10 \mathrm{mM} \mathrm{NaCl}, 1.5 \mathrm{mM} \mathrm{MgCl}, 10 \mathrm{mM}$ Tris-HCl, pH 7.5) and homogenized using Potter-Elvehjem homogenizer with a Teflon pestle. Following homogenization, cells were subjected to differential centrifugation in order to obtain nuclear/whole cell, crude mitochondrial, and microsomal fractions. Supernatants were collected in order to obtain cytosolic/microsomal fractions. The crude mitochondrial fraction in MS homogenization buffer (210 Mm mannitol, 70 $\mathrm{mM}$ sucrose, $5 \mathrm{mM}$ Tris- $\mathrm{HCl}, 1 \mathrm{mM}$ EDTA, $\mathrm{pH}$ 7.5) was subjected to sucrose density gradient centrifugation (1.0 M sucrose or $1.5 \mathrm{M}$ sucrose, $10 \mathrm{mM}$ Tris-HCl, $1 \mathrm{mM}$ EDTA, pH 7.5) to obtain pure mitochondria. Cytoplasmic fractions were ultracentrifuged at $100,000 \times \mathrm{g}$ for $1 \mathrm{~h}$ at 4 으 $\mathrm{C}$ to isolate cytosolic and microsomal fractions. 


\subsubsection{Biochemical procedures}

\subsubsection{Preparation of whole cell lysates from adherent cells}

For the preparation of whole cell lysates, cell pellets from previously harvested adherent cells were resuspended on ice in $50-150 \mu \mathrm{L}$ of lysis buffer (1.5\% N-dodecyl $\beta$-D-maltoside in PBS $1 \mathrm{x}$ supplemented protease and phosphatase inhibitor), depending on pellet size. Afterwards, the cell suspension was lysed by rotation at $4{ }^{\circ} \mathrm{C}$ for 30 minutes and centrifuged during 20 minutes at $16,000 \mathrm{rpm}$ in order to obtain the cell homogenate. The supernatant (whole cell lysate) was collected and the protein concentration was measured by the Bradford Assay. The lysates were either used for further experiments or aliquoted and frozen at $-80^{\circ} \mathrm{C}$ in order to avoid freezethaw cycles.

\subsubsection{Protein concentration determination using Bradford assay}

For the determination of protein concentrations, Protein Assay Dye Reagent Concentrate (BioRad) was used accordingly manufacturer's protocol. For this purpose, protein concentration of cell lysates and standards using bovine serum albumin (BSA) was determined by mixing, $800 \mu \mathrm{L}$ of ${ }_{d d} \mathrm{H}_{2} \mathrm{O}, 1 \mu \mathrm{L}$ of sample or standard and $200 \mu \mathrm{L}$ of protein assay dye reagent concentrate in a glass tube and vortexed briefly. Afterwards, the tubes were incubated during 5 minutes at room temperature in the dark and transferred into plastic cuvettes. The absorbance at $595 \mathrm{~nm}$ was measured using a GeneQuant 1300 spectrophotometer. The protein concentration of the cell lysates was calculated based on the standard calibration curve.

\subsubsection{SDS-PAGE and Immunoblotting}

After protein quantification, equal amount of protein from cell lysates was mixed with homemade $6 x$ SDS loading buffer and with the appropriate volume of ${ }_{d d} \mathrm{H}_{2} \mathrm{O}$ to load the same amount of protein in each well and boiled for 5 minutes at 95으. These steps allow for protein denaturation and therefore loss of quaternary, tertiary and secondary protein structure. Moreover, proteins obtained a uniform negative charge, which masks the intrinsic charges on the amino acids side-chain. Consequently, after this point, proteins can be separated solely as a function of their molecular size by SDS-PAGE (Gallagher and li, 2006). To accomplish that, samples (10 to $25 \mu \mathrm{g}$ of protein) were loaded in a discontinuous vertical polyacrylamide gel electrophoresis system. Polyacrylamide gels were formed by copolymerization between 
acrylamide and bis-acrylaminde through a vinyl addition polymerization reaction initiated with the addition of ammonium persulfate (APS) and catalysed by tetramethylethylenediamine (TEMED). To run the SDS-PAGE, the Mini Protean Tetra System (Bio-Rad) was used filled with $1 \mathrm{x}$ running buffer and connected to a PowerPac Basic Power Supply (Bio-Rad) outputting an initial constant amperage of $20 \mathrm{~mA}$ per gel and switched to constant 30-40 mA when samples were in the resolving gel. Separation was carried out at room temperature and until the front of the run reached the bottom end of the gel. Note that in every gel, a molecular weight standard (PageRuler plus Prestained ladder) was included to allow molecular weight estimation.

Once protein separation was complete, Western blot was carried according to protocols described by (Gallagher and li, 2006). Briefly, polyvinylidene fluoride (PVDF) membranes were pre-activated ( 1 minute in methanol, washed 1 minute with ddH2O followed by 15 min in transfer buffer $1 \mathrm{x}$ ). For the protein transfer, gels were placed in a 'transfer sandwich' (filter paper-gel-membrane-filter paper), cushioned by wet sponges and pressed together by a support grid/cassette. The supported gel sandwich was then placed in the transfer apparatus in a tank filled with $1 \mathrm{X}$ transfer buffer while ensuring the correct orientation (bottom half towards cathode). A cold ice pack was also placed in the tank to avoid excessive heating of the transfer buffer. Protein transfer was performed at a constant voltage $(100 \mathrm{mV})$ during $90 \mathrm{~min}$ with a PowerPac ${ }^{\mathrm{TM}}$ Basic Power Supply (Bio-Rad). Once protein transfer was complete, the membranes were blocked in blocking buffer with mild shaking for 1 hour at RT, to avoid non-specific binding. The membranes were then washed three times in 1X TBST for 5 minutes and labeled with primary antibodies (diluted in blocking buffer) by overnight incubation at $4{ }^{\circ} \mathrm{C}$. On the next day, membranes were washed three times for $10 \mathrm{~min}$ each with $1 \mathrm{X}$ TBS-T and incubated with appropriate HRP-conjugated secondary antibodies diluted in blocking buffer at RT for 1 hour and washed three more times in 1X TBST for 10 minutes. Signals representing proteins by chemiluminescence were detected by draining excess $1 \mathrm{X}$ TBST on membranes, incubating membranes in Luminata Western HRP substrate for 3 minutes and developing signals on medical X-ray films using the AGFA Curix 60 processor. The films were scanned with the Epson Perfection V850 Pro Scanner. Band density quantification was determined using ImageJ after subtraction of background and normalized against loading controls (HPRT).

\subsubsection{Immunoprecipitation}

For immunoprecipitation, cells were used at approximately $80 \%$ of confluence. For collection and lysis, cells were harvested using cell scraper, lysis and protein extraction was performed as 
described before (see 3.2.3.1.). After protein quantification by Bradford assay, $500 \mu \mathrm{g}$ of protein in $500 \mu \mathrm{l}$ of WCE buffer was prepared. Afterwards, protein G agarose (30 $\mu \mathrm{l}$ per IP) was equilibrated with $500 \mu \mathrm{l}$ PBS1x prior to adding it to lysate and centrifugated at 2000xg for 2 min. After centrifugation, supernatant was removed and $500 \mu$ l of the prepared cell lysate (containing $500 \mu \mathrm{g}$ of protein) was added. The antibodies ( $5 \mu \mathrm{l}$ of antibody per IP) were added to the previous solution and incubated at $4{ }^{\circ} \mathrm{C}$ overnight with gentle rotation.

On the next day, centrifugation at 2000xg for 2 minutes was performed and the supernatant discarded. In the next step, $500 \mu \mathrm{l}$ of WCE was added and incubated during 5 minutes at $4^{\circ} \mathrm{C}$ with rotation, followed by centrifugation at $2000 x g$ for 2 minutes. This was repeated for at least 4 times and in the last step most of the supernatant was removed and approximately $40 \mu \mathrm{l}$ of SDS loading dye was added to the beads. The samples were boiled for 5 minutes at $95^{\circ} \mathrm{C}$ and centrifuged at maximum speed (16000xg) for 1 minute and $10 \mu \mathrm{l}$ of the supernatant was analyzed by western blotting.

\subsubsection{Evaluation of oxygen consumption}

Respirometry of cultured cells in this study was evaluated using the Seahorse XF96 Extracellular Flux Analyzer (Seahorse Bioscience). The $\mathrm{XF}^{\mathrm{e}} 96$ extracellular flux assay kit was used following manufacturer's protocols. Cells were seeded in the XF96 cell culture plate in $200 \mu \mathrm{L}$ normal growth medium per well and cultured overnight. In the same day, it was prepared the the $\mathrm{XF}^{\mathrm{e}}$ 96 sensor cartridge plate by adding $200 \mu \mathrm{L}$ of XF calibrant to it and incubated in a non-CO2 incubator at $37^{\circ} \mathrm{C}$ overnight. Next day, XF medium was prepared by supplementing with $25 \mathrm{mM}$ glucose and $\mathrm{pH}$ adjusted to 7.4 and the previously cultured cells were washed with PBS $1 \mathrm{x}$ and the medium was replaced with $180 \mu \mathrm{L}$ of warm XF medium and incubated at $37^{\circ} \mathrm{C}$ for 1 hour without CO2. During this incubation time, the sensor cartridge was loaded in the indicated ports with different compounds which were prepared in warm XF medium. The compounds were $10 \mu \mathrm{M}$ Oligomycin $(20 \mu \mathrm{L}$ per well loaded in Port A), $20 \mu \mathrm{M}$ FCCP $(22 \mu \mathrm{L}$ per well loaded in Port B) and $10 \mu \mathrm{M}$ each of Rotenone and Antimycin ( $25 \mu \mathrm{L}$ per well loaded in Port $\mathrm{C})$. Afterwards, the sensor cartridge plate was loaded and calibrated in the XF96 extracellular Flux Analyzer. Once the calibration was completed, the XF96 cell culture plate was loaded into the XF96 extracellular Flux Analyzer at $37^{\circ} \mathrm{C}$ and a MitoStress Test was run using the $\mathrm{XF}^{\mathrm{e}}$ analyzer software. All results were averages of at least technical triplicates of three biological replicates. 


\subsubsection{Flow cytometry}

\subsubsection{Mitochondrial membrane potential}

Mitochondrial membrane potential was determined by flow cytometry using JC1 dye (Molecular Probes) according to manufacturer's recommendation. Briefly, $5 \mathrm{mM}$ of JC1 stock was prepared by resuspending in DMSO. The JC1 working solution $(20 \mu \mathrm{M})$ was prepared in warm DMEM medium. Previously, cultured cells were washed with warm PBS $1 x$, stained by adding JC1 working solution to cells, and incubating at $37^{\circ} \mathrm{C}$ for 20 minutes. Afterwards, the excess of dye was washed off the cells twice with warm PBS $1 x$ and cells were harvested by trypsinization as described before. Then, cells were centrifuged at $1500 \mathrm{rpm}$ for 5 minutes and resuspended in $1000 \mu \mathrm{L}$ of cold PBS 1x. Cell suspension was transferred to FACS tubes along with unstained cells, stained test samples and positive controls (treated with $10 \mu \mathrm{M}$ of FCCP) and mitochondrial membrane potential was determined by flow cytometry. The results were analyzed using FlowJo v10 software.

\subsubsection{Measurement of mitochondrial superoxide levels}

The MitoSOX Red mitochondrial superoxide indicator (Molecular Probes) was used in order to measure mitochondrial superoxide levels by flow cytometry according to manufacturer's protocol. For this purpose, $5 \mathrm{mM}$ MitoSOX concentration stock was prepared by resuspending

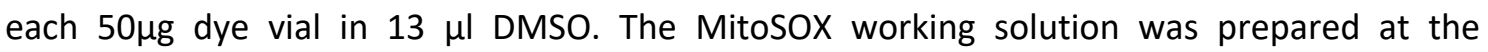
concentration of $5 \mu \mathrm{M}$ with warm PBS 1x supplemented with $0.5 \mu \mathrm{M}$ of glucose. The cultured cells were washed with PBS $1 x$ and stained with MitoSOX working solution by incubating at 37 ${ }^{\circ} \mathrm{C}$ for 15 minutes. Following staining, cells were washed with warm PBS $1 \mathrm{x}$ and harvested by trypsinization as described before. Afterwards, cells were washed twice with PBS $1 \mathrm{x}$ and centrifuged during 5 minutes at $1500 \mathrm{rpm}$. The cell pellet was resuspended in $500 \mu \mathrm{L}$ of PBS $1 \mathrm{x}$ supplemented with $0.5 \mu \mathrm{M}$ of glucose. Cell suspension was transferred to FACS tubes along with unstained cells, stained test samples and mitochondrial superoxide levels were determined by flow cytometry. As positive controls, cells were treated with $100 \mu \mathrm{M} \mathrm{H}_{2} \mathrm{O}_{2}$ or $100 \mu \mathrm{M}$ Antimycin for 20 minutes at $37^{\circ} \mathrm{C}$. The results were analyzed using FlowJo v10 software. 


\subsubsection{Immunocytochemistry}

Cells seeded in 12-well-plates containing coverslips were washed with PBS $1 x$ and fixed using $4 \%$ of PFA. After that, the PFA was removed and the coverslips were washed 3 times with PBS $1 \mathrm{x}$. Cells were blocked and permeabilized with $500 \mu \mathrm{L}$ of blocking solution during $1 \mathrm{~h}$ at room temperature with mild shacking. Then, the coverslips were incubated with primary antibodies overnight at $4^{\circ} \mathrm{C}$. The next day, the antibodies were removed, and the coverslips were washed thrice with PBS $1 x$ for 10 minutes. After the wash, the coverslips were incubated with secondary antibodies (1:200) for an hour at $\mathrm{R} / \mathrm{T}$ in the dark. When the incubation was completed, the antibody was removed, and the coverslips washed three times again with PBS for 10 minutes. The coverslips were mounted on slides using Mowiol, dried, sealed with nail polish and stored in the dark at $4^{\circ} \mathrm{C}$ until imaged.

\subsubsection{Confocal microscopy and image analysis}

HeLa RC-kd cells and MEFs were plated and transiently transfected as described before on coverslips in 12 well-plates at normal growth conditions and fixed after 24 hours. Images were obtained using a Zeiss LSM 800 Laser Scanning Microscope with Airyscan. Images were acquired with the same parameters throughout all experiments. All settings were saved and applied to subsequent imaging sessions for comparable groups. Post-acquisition image analyses were carried out with ZEN software (Zeiss) and ImageJ.

\subsubsection{Electron microscopy and image analysis}

Cells seeded on $24 \mathrm{~mm}$ round glass coverslips were fixed with $2.5 \%$ (vol/vol) glutaraldehyde in $0.1 \mathrm{M}$ sodium cacodylate during $1 \mathrm{~h}$ at room temperature. After washing with $0.1 \mathrm{M}$ cacodylate buffer, cells were post-fixed with $1 \%$ of $\mathrm{OsO}_{4}$ solution and $1.5 \%$ of ferrocyanide in $0.1 \mathrm{M}$ of cacodylate during $1 \mathrm{~h}$ at room temperature. The samples were then washed three times in $0.1 \mathrm{M}$ of cacodylate buffer followed by a further three times washes in Millipore water and then incubated in $1 \%$ aqueous uranyl acetate at $4{ }^{\circ} \mathrm{C}$ overnight. Then, cells were rinsed with Millipore water, dehydrated with ethanol and infiltrated in Epon using 1:1 (v/v) resin and anhydrous ethanol for $1 \mathrm{~h}$. Additionally, two steps of $100 \%$ resin during $1 \mathrm{~h}$ were performed. After, the cells were incubated at $45^{\circ} \mathrm{C}$ for one overnight followed by a second overnight at $60{ }^{\circ} \mathrm{C}$. Thin sections 
were obtained and imaged using a JEM 1011 transmission electron microscope (JEOL) operated at $80 \mathrm{kV}$, equipped with a Gatan Orius $1200 \mathrm{~A}$ camera using the Digital Micrograph software package. Two independent experiments were performed, and each sample was labeled with a numerical code. The electron microscopy images were collected blinded at 4000x or higher magnification and then imported into ImageJ (National Institutes of Health) for further analysis. Morphometric analysis was carried out at least in 100 images per condition with $>5$ mitochondria per image. The analysis of MERCs number, mitochondrial perimeter and length, were also carried out blind, using ImageJ software and setting $30 \mathrm{~nm}$ as the maximum distance from any segment of an ER tubule to a mitochondrion in order to be considered a contact site. In ImageJ software, the mitochondrial perimeter for each clearly defined mitochondrion was outlined by drawing a freehand line and measured. These values were then averaged to get the average mitochondrial perimeter for a given sample. To measure the length of the contact, it was drawn a freehand line from the beginning to the end of the mitochondrion-ER contact that was considered before. These values were then averaged to get the average length of contact sites for a given sample.

\subsubsection{Mitochondrial calcium measurement}

Mitochondrial $\mathrm{Ca}^{2+}$ was measured using $4 \mathrm{mtD} 3 \mathrm{cpv}$ (mitochondrial cameleon). Cells seeded on $25 \mathrm{~mm}$ round glass coverslips were transiently transfected with $1 \mu \mathrm{g}$ of $4 \mathrm{mtD} 3 \mathrm{cpV}$ using Fugene 6 Transfection Reagent (Promega) according to the manufacturer's instructions and as described before (see 2.2.1.3.). The experiments were performed $48 \mathrm{~h}$ after transfection. On the experimental day, cell-containing glass coverslip was inserted into the microscopy chamber and the growth medium replaced by Ringer's buffer $(55 \mathrm{mM} \mathrm{NaCl}, 4.5 \mathrm{mM} \mathrm{KCl}, 10 \mathrm{mM}$ glucose, 5 $\mathrm{mM}$ HEPES, $2.75 \mathrm{mM} \mathrm{MgCl}_{2}, 0.5 \mathrm{mM} \mathrm{CaCl}_{2}, \mathrm{pH} 7.437^{\circ} \mathrm{C}$ ). The image acquisition was obtained with a Cell Observer High Speed (Zeiss) microscope equipped with 40x oil Fluar (N.A. 1.3) objective. The experiment started with the measurement of the basal level of $\mathrm{Ca}^{2+}$ for at least 40 cycles and afterwards mitochondrial $\mathrm{Ca}^{2+}$ uptake was induced by $1 \mathrm{mM}$ histamine. Data analysis was performed with the software provided by the microscope supplier and to calculate FRET ratios, background intensity was subtracted. 


\subsubsection{Statistical analyses}

The results were analysed using Microsoft Excel 2013 and GraphPad Prism version 6. For Western blotting, all data points represent the mean of at least three independent biological replicates. Error bars represent standard errors of the mean unless otherwise stated. For confocal images, typically at least 30 cells were analyzed from at least three independent experiments; the data indicate average and the error bars represent the standard deviation. Differences of means were considered statistically significant for parametric data if they passed thresholds calculated as Student's $t$-test for two-parameter comparisons. For electron microscopy data, all data points represent the mean of at least three independent biological replicates. Error bars represent standard errors of the mean. Differences of means were considered statistically significant based on Mann-Whitney test. These were indicated as $p<$ $0.05(*), \mathrm{p}<0.01(* *)$ or $\mathrm{p}<0.001(* * *)$. 


\section{RESULTS}

\subsection{Loss of AMPK activity results in increased endoplasmic reticulum- mitochondria contact sites}

\subsubsection{Mitochondrial function is compromised in AMPK defective cells}

Mitochondria are the major cellular ATP suppliers, promoting the maintenance of energy homeostasis, and therefore it is important for the cells to adjust when mitochondria are not performing optimally (Mihaylova and Shaw, 2011; Toyama et al., 2016). The AMPK is involved in several aspects of mitochondrial biology and homeostasis (Herzig and Shaw, 2018; Kahn et al., 2005; Toyama et al., 2016). AMPK signaling is one of the major metabolic pathways activated after acute mitochondrial insults, restoring the energy levels by inhibiting ATP-consuming pathways or by stimulating catabolic ATP- generating processes (Fernández-Mosquera et al., 2017; Toyama et al., 2016). In this sense, in this project we explored if there is an association between mitochondrial signaling pathways, which are activated by mitochondrial stress, and the regulation of MERCs. For this purpose, mouse embryonic fibroblasts lacking the two catalytic subunits of AMPK, $\alpha 1$ and $\alpha 2$ (AMPK $\alpha 1 \alpha 2$ double knock-out, henceforth AMPK-DKO) were used to evaluate if AMPK is involved in the regulation of MERCs, given its essential role in responding to mitochondrial malfunctions as described above (Laderoute et al., 2006). In order to demonstrate that both AMPK catalytic subunits were lacking, we measured AMPK activity by assessing protein levels of AMPK (and phospho-T172 of AMPK, a marker of active AMPK), and phosphorylation of ACC pSer ${ }^{79}$, one of the AMPK targets, by western blot. As expected, we observed no phosphorylation of AMPK-T172 and ACC pSer ${ }^{79}$, indicating no AMPK activity in AMPK-DKO (Figure 8a). Moreover, mitochondrial function was also analyzed through the evaluation of different parameters, such as, mitochondrial membrane potential, oxygen consumption and ROS production. Mitochondrial oxygen consumption rate (OCR) was measured under basal conditions and after inhibition or uncoupling oxidative phosphorylation (maximal OCR). The AMPK-DKO MEFs showed reduced basal and maximal OCR (Figure 8b-d). Additionally, ATP-linked respiration, as well as spare respiratory capacity were reduced in AMPK-DKO cells when compared with WT cells (Figure 8e and 8f). Taken together, these results show an impairment of mitochondrial function in AMPK-DKO cells, as expected. 
a

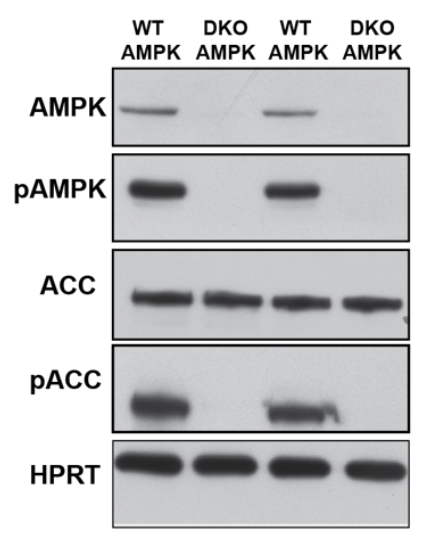

C

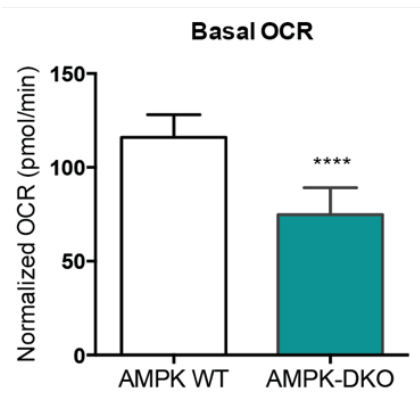

e

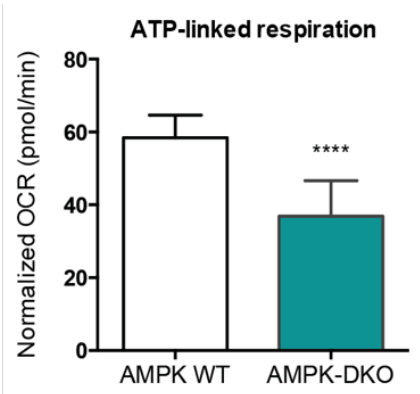

b

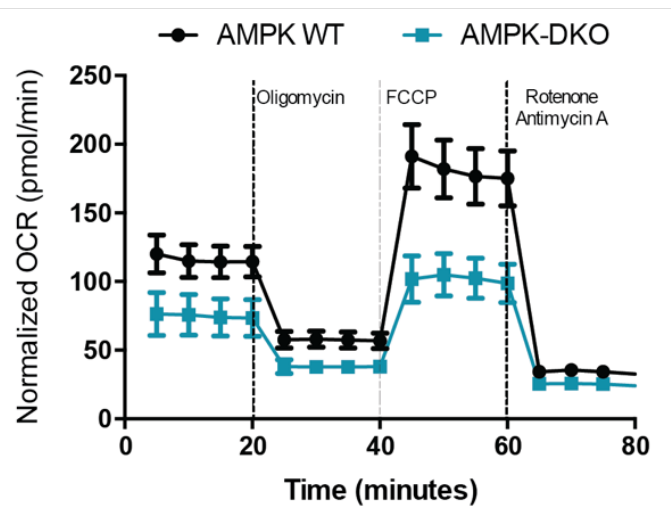

d

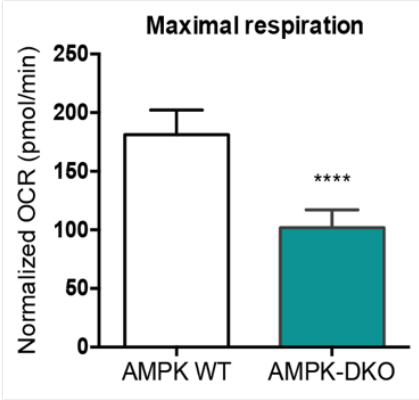

$\mathbf{f}$

Spare respiratory capacity

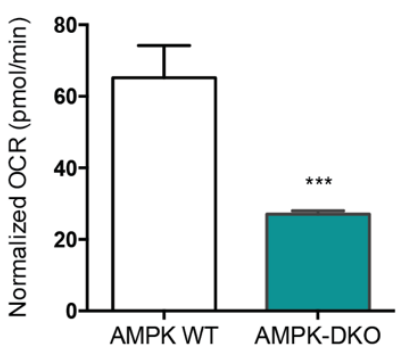

Figure 8 | Mitochondrial function is compromised in a defective AMPK cell model. (a) Representative immunoblots of AMPK substrates (b) Mitochondrial oxygen consumption rate as assessed by Real Time Respirometry (c) Reduced basal mitochondrial OCR in AMPK-DKO MEFs (d) Reduced maximal mitochondrial OCR in AMPK-DKO MEFs measured by Real Time Respirometry (e) ATP-linked respiration reduction in AMPK-DKO (f) Reduced spare respiratory capacity. Data are representative for three independent measurements. Graphs are mean \pm SEM for the indicated number of sample replicates and the differences were significant based on the $t$ test, $* * * \mathrm{p} \leq 0.001$ and $* * * * \mathrm{p} \leq 0.0001 ;$ SEM: standard error of the mean. 
Mitochondrial membrane potential was measured by flow cytometry using JC-1 dye. This probe is a cationic carbocyanine dye that accumulates in mitochondria, and exhibits fluorescence emission at two typical wavelengths: (i) red fluorescent J-aggregates (emission maximum at 590 $\mathrm{nm}$ ) at high mitochondrial concentrations reflecting higher mitochondrial potential; and (ii) green fluorescent J-monomers (emission maximum at $\sim 529 \mathrm{~nm}$ ) at low mitochondrial concentrations indicating loss of membrane potential (Chazotte, 2011; Elefantova et al., 2018). In this experiment, subpopulations can be obtained accordingly with the different intensities in both channels. Therefore, healthy non-apoptotic cells will be detected in both red and green channels, corresponding to the Q2 subpopulation. However, cells with mitochondrial dysfunction will have more green intensity, but less red fluorescence, represented as the Q3 subpopulation in our analysis. From our results, we observed that $12.4 \%$ of AMPK-DKO and only $2.25 \%$ of WT cells were in Q3 population, this increase suggests low mitochondrial membrane potential $(\triangle \Psi \mathrm{m})$ in AMPK-DKO cells (Figure 9a and 9c). As a positive control, FCCP was used to demonstrate increased population of cells with mitochondrial dysfunction (increased green fluorescence). This chemical causes a quick mitochondrial membrane depolarization, reducing the red fluorescence signal and reflecting an increase of $56.9 \%$ of cells with low $\Delta \Psi \mathrm{m}$ (Figure 9b). 
a

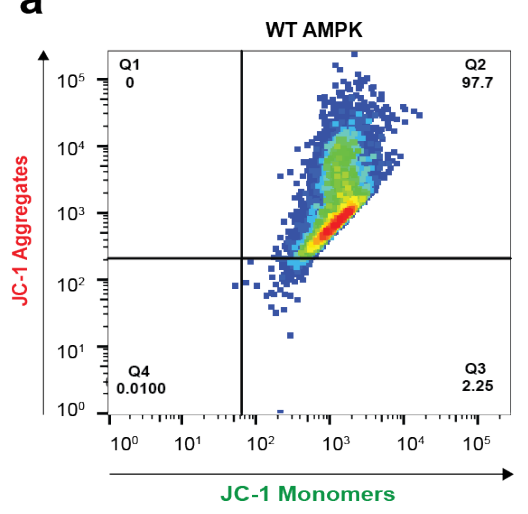

C

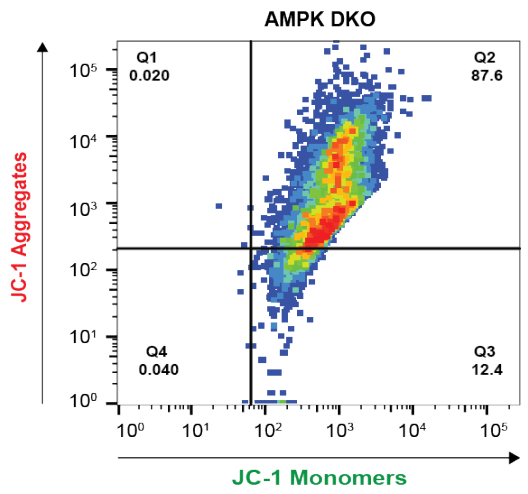

b

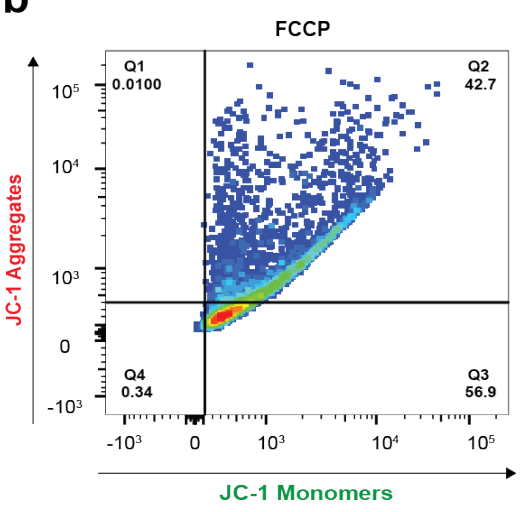

d

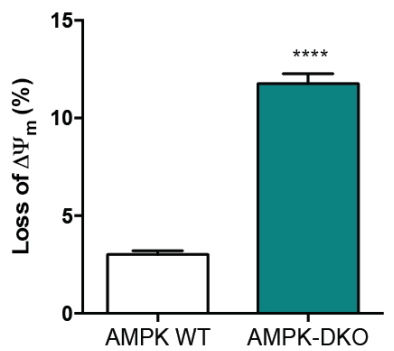

Figure 9 | AMPK-DKO cells have lower mitochondrial membrane potential. (a) JC1-monomers vs. JC1-aggregates plot for WT cells with JC-1. (b) JC1-monomers vs. JC1-aggregates plot for cells with JC-1 and FCCP, as a positive control. (c) JC1-monomers vs. JC1-aggregates plot for AMPK-DKO cells with JC-1 (d) Percentage of WT and AMPKDKO cells with positive green signal, represented as Q3 subpopulation. Data are representative for three independent measurements. Graph represents mean \pm SEM for the indicated number of sample replicates and the differences were significant based on the t-test, $* * * * p \leq 0.0001$; SEM: standard error of the mean.

The ETC is a major source of cellular ROS due to the inefficient reduction of molecular oxygen in the respiratory chain (Rabinovitch et al., 2017; Valko et al., 2007). Therefore, mitochondrial ROS measurement is often used as an indirect parameter to investigate mitochondrial function. In this way, mitochondrial ROS production was analyzed by flow cytometry using MitoSOX probe, but no significant differences were observed between WT and AMPK-DKO cells (Figure 10a and 10b). 
a

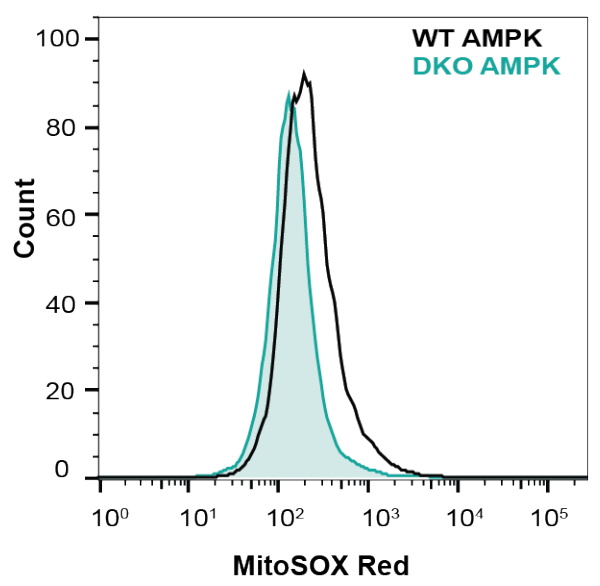

b

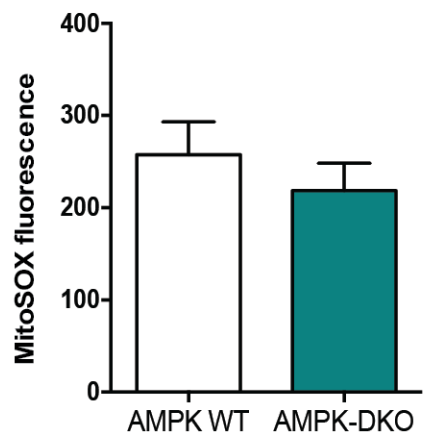

Figure 10 | MitoSox fluorescence in WT and AMPK-DKO cells, representing mitochondrial superoxide levels. (a) Data are representative for three independent measurements. (b) Graph represents mean \pm SEM for the indicated number of sample replicates; SEM: standard error of the mean.

Altogether, these data show that in AMPK-DKO model there is an impairment of mitochondrial function. Therefore, we decided to investigate if the mitochondrial morphology was also affected in the AMPK-DKO cells. For this purpose, cells were immunostained with TOM20, a marker for mitochondria, and several parameters were analyzed, such as mitochondrial area and perimeter. From our analysis, no alterations were observed in mitochondrial morphology in AMPK-DKO in all parameters analyzed (Figure 11a-c). 
a
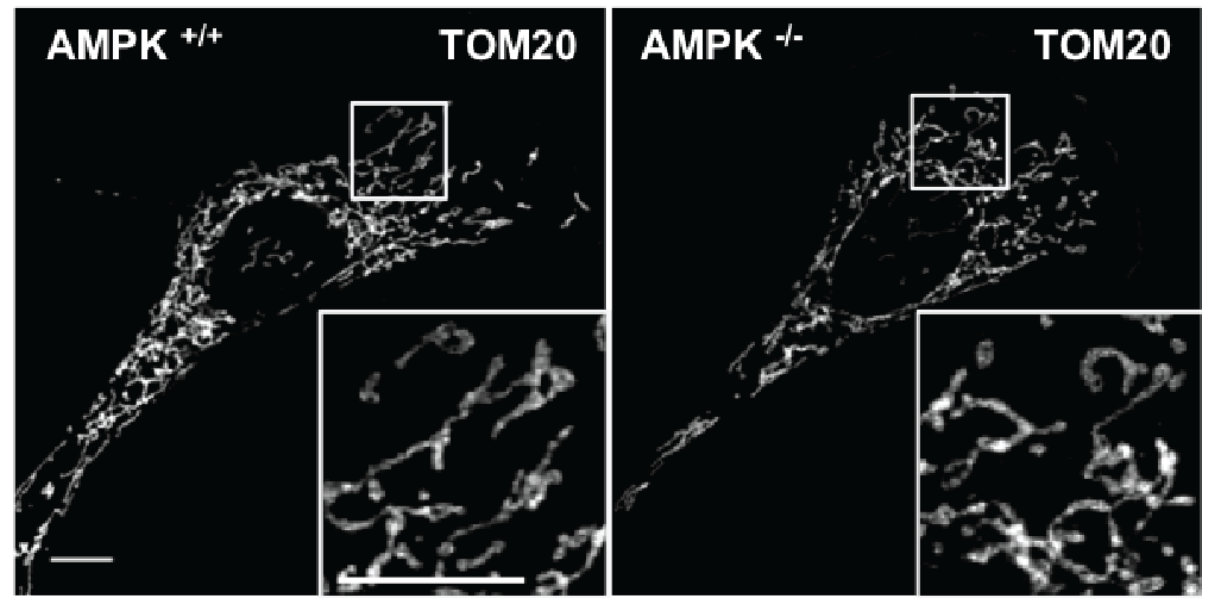

b

C

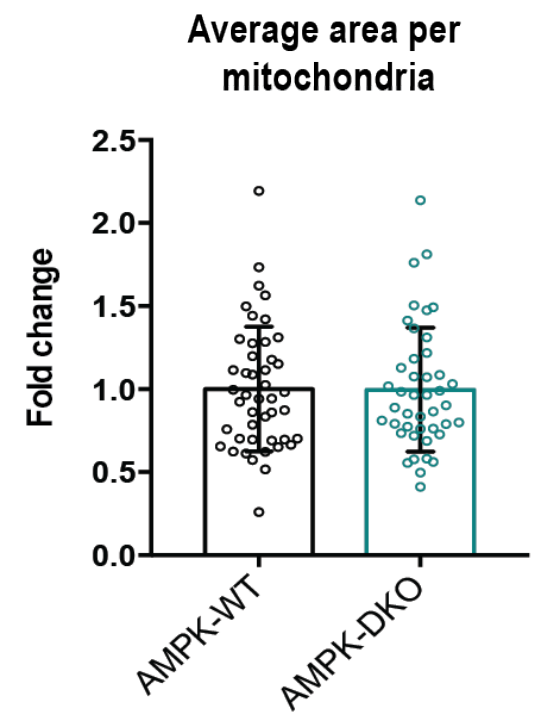

\section{Average perimeter per mitochondria}

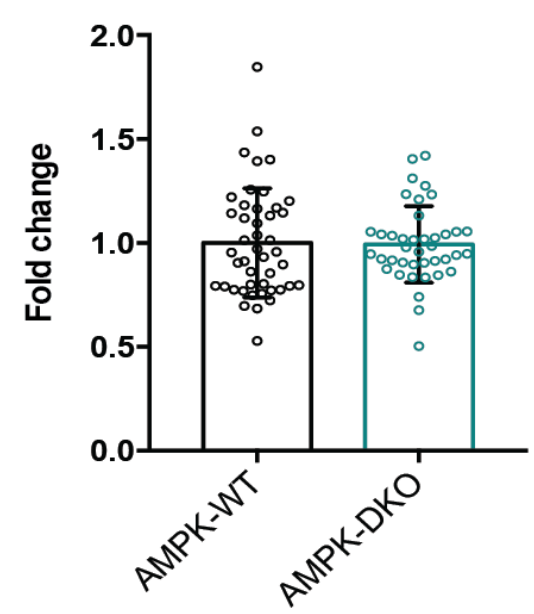

Figure 11 | Mitochondrial morphology is not altered in AMPK-DKO. (a) Representative TOM20 immunostainings of WT and AMPK-DKO (Scale bar $10 \mu \mathrm{m}$ ). ( $\mathbf{b}$ and $\mathbf{c}$ ) Respective mitochondrial morphology analysis of cells in $\mathbf{b}$ average area per mitochondria and $\mathbf{c}$ average perimeter per mitochondria. Data represent mean \pm SD of three independent experiments.

\subsubsection{Absence of AMPK activity enhances ER-mitochondria communication in} mouse embryonic fibroblasts

ER and mitochondria contact sites are localized in specific regions of the cell, when the organelles are in close proximity, with an inter-membrane distance of 10-30 nm (Csordás et al., 
2006). Therefore, electron microscopy (EM) is required in order to achieve the resolution needed to analyze such close contacts. Furthermore, several parameters, such as number of contacts and extension, morphological changes of the organelles, can be provided by EM (Csordás et al., 2018; Filadi et al., 2018). In this manner, electron micrographs of these cells were prepared and used to quantify the number and extension of MERCs (Figure 12a). For this purpose, the distance of $30 \mathrm{~nm}$ between both organelles was a key criterion to consider organelle proximity as a contact site. Representative images are shown in Figure 12a, for which 281 contacts ( $n=30$ cells) were analyzed in WT cells and 517 contacts in AMPK-DKO cells $(n=29$ cells). From the systematic quantification of MERCs, we observed a robust increase of the number of MERCs in AMPK-DKO cells (Figure 12b). Additionally, the average perimeter of the mitochondria involved in contact sites was decreased in AMPK-DKO cells, suggesting that the increase of the number of MERCs is not a consequence of bigger mitochondria (Figure 12c).

a
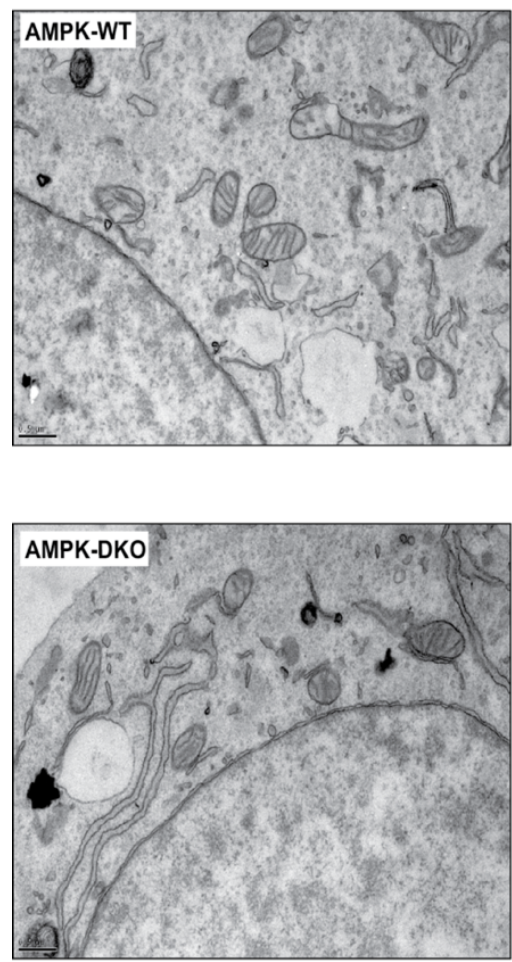
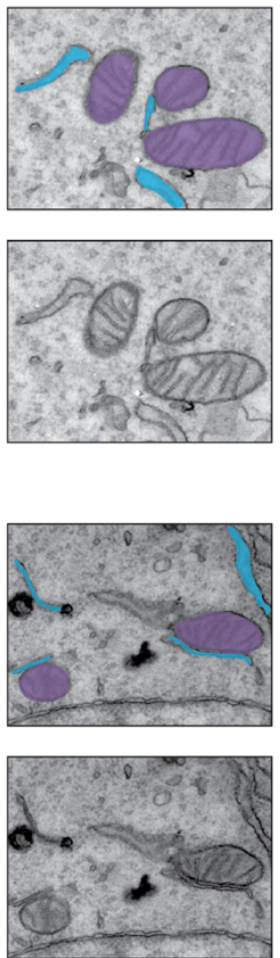

b

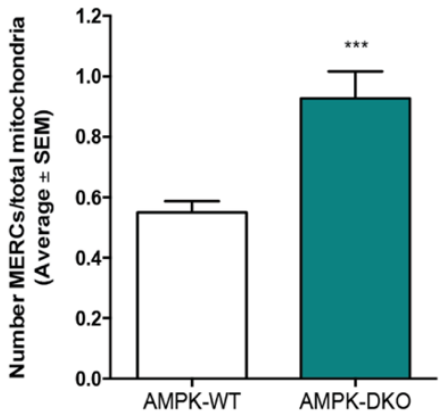

C

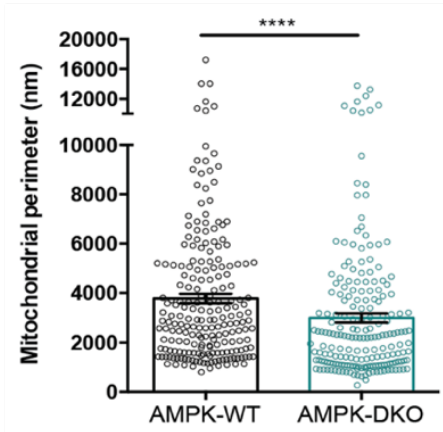

Figure 12 | AMPK is a negative regulator of ER-mitochondria contact sites. (a) Representative EM images of WT and AMPK-DKO MEF (Scale bars, $0.5 \mu \mathrm{m}$ ) (b) Quantification of the number of ER-mitochondria contact sites, in which the organelles are separated by less than $30-\mathrm{nm}$. Data represent mean \pm SEM of two independent experiments ( $\mathrm{n}>300$ mitochondria per experiment). (c) Morphometric analysis of mitochondrial perimeter involved in contact sites. Data represent mean \pm SEM of two independent experiments $(n>300$ mitochondria per 
experiment). The differences were significant based on Mann-Whitney test; ${ }^{* *}{ }^{*}<<0.001 ;{ }^{* * * *} \mathrm{P}<0.0001 ; \mathrm{SEM}$ : standard error of the mean.

Regarding the length of MERCs, no difference was observed in the average length between AMPK-DKO and WT cells (Figure 13a); however longer contact sites were observed in AMPKDKO cells (Figure 13b). Taken together, these results suggest that absence of AMPK activity is associated with increased number of MERCs.

a

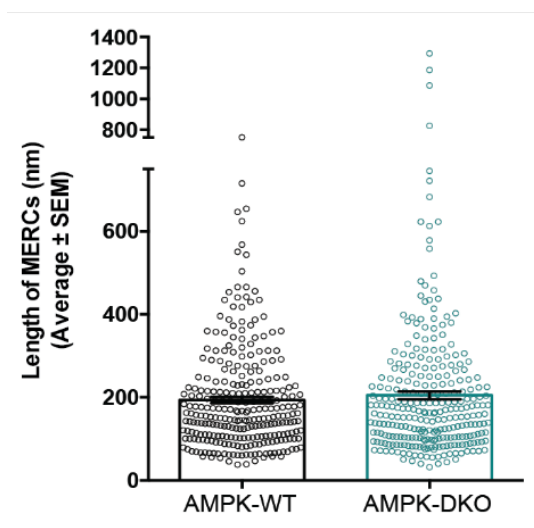

b

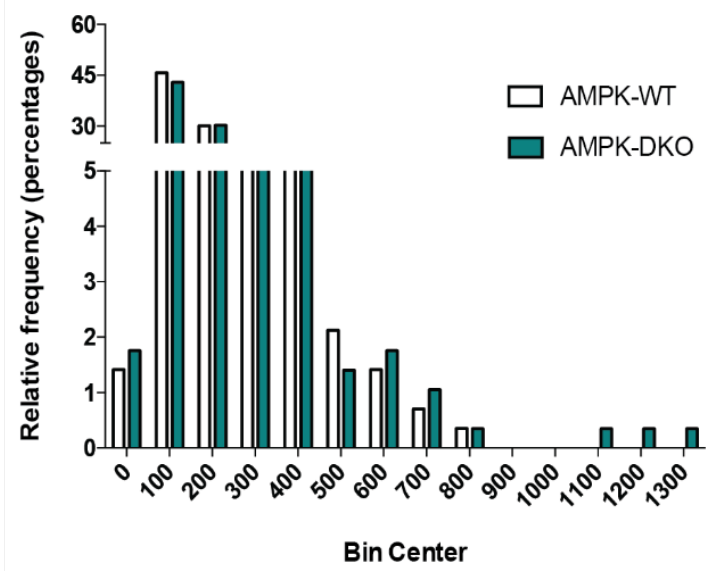

Figure 13 | AMPK -DKO cells have longer contact sites than WT AMPK cells. (a) Morphometric analysis of ER length located at less than 30-nm from mitochondria. Data represent mean \pm SEM of two independent experiments ( $n>300$ mitochondria per experiment) (b) Histogram reporting lengths quantified in (a). SEM: standard error of the mean.

To further validate this result, we transfected cells with GFP targeted to the cytoplasmic side of the ER membrane (EGFP-ER) and immunostained the cells with TOM2O for mitochondria and obtained confocal images of the cells (Figure 14a). The analysis showed that the overlap between the two signals was higher in AMPK-DKOs (Figure 14b). This result suggests an increased amount of mitochondria-ER contact sites in the AMPK-DKO cells, in agreement with our electron microscopy data. 
a

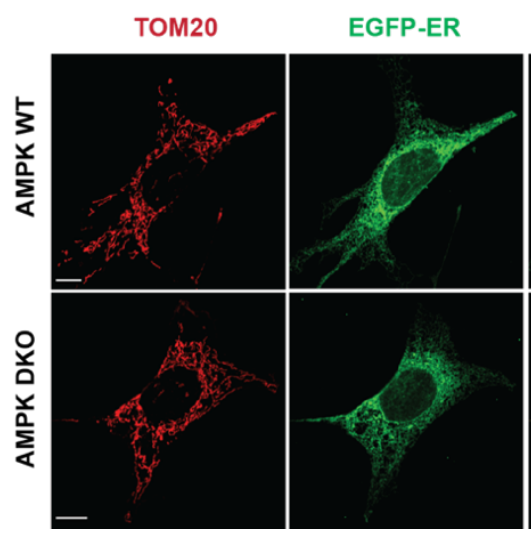

b

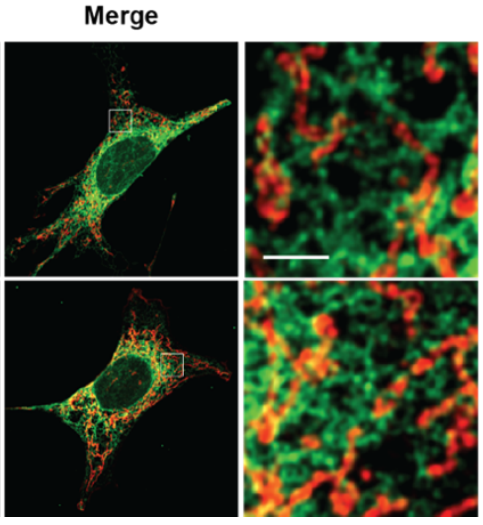

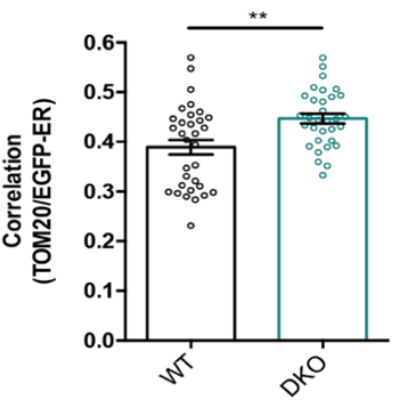

Figure 14 | Increased overlap between mitochondria and ER in AMPK-DKOs. (a) Representative confocal images of MEFs of the indicated genotype transiently transfected with an ER marker (green) and immunostained with TOM20 (red) for mitochondria (Scale bar $10 \mu \mathrm{m}$ ). (b) Quantification of the TOM20 (mitochondria) and TM-Sac1GFP (ER) colocalization is shown in (b). Graph represents mean \pm SD of three independent experiments and the differences were significant based on the t-test; $* * \mathrm{P}<0.01$.

\subsubsection{Basal mitochondrial calcium is increased in AMPK-DKO MEFs}

The electron microscopy approach allowed us to investigate the structure of MERCs, however this does not take into consideration the dynamics of ER-mitochondria contacts. Therefore, as a functional readout we focused on one of the hallmarks of MERCs, which is calcium transfer between the organelles (Patergnani et al., 2011). Since we observed an enhancement of the number of contact sites in our model of AMPK-DKO, we hypothesized that this effect consequently could affect calcium trafficking mediated by MERCs. Thus, we employed a FRET probe, whose emission shifts in a $\mathrm{Ca}^{2+}$-dependent manner, as illustrated in Figure 15 (Gibhardt et al., 2016; Palmer and Tsien, 2006; Palmer et al., 2006). Furthermore, this strategy is advantageous, since it is possible to specifically target this probe to mitochondria, and therefore specifically follow mitochondrial $\mathrm{Ca}^{2+}$ levels (Gibhardt et al., 2016). 

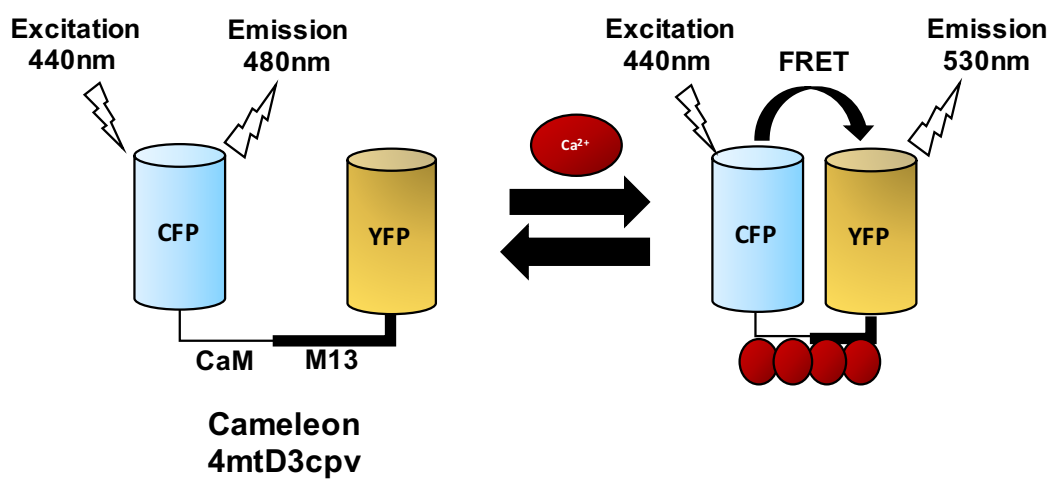

\section{4mtD3cpv}

Figure 15 | Schematic illustration of the FRET sensor $4 \mathrm{mtD} 3 \mathrm{cpV}$, targeted to mitochondria and allowing mitochondrial $\mathrm{Ca}^{2+}$ measurement. This emission shifts in a $\mathrm{Ca}^{2+}$-dependent manner $\left(\mathrm{K}_{d}=0.6 \mu \mathrm{M}\right)$.

For this purpose, AMP-WT and AMPK-DKO cells were transiently transfected with $4 \mathrm{mtD} 3 \mathrm{cpv}$ (mitochondrial cameleon) followed by live imaging. The basal mitochondrial $\mathrm{Ca}^{2+}$ measured were increased in AMPK-DKO cells, which is consistent with the increased number of MERCs observed previously (Figure 16b).

a

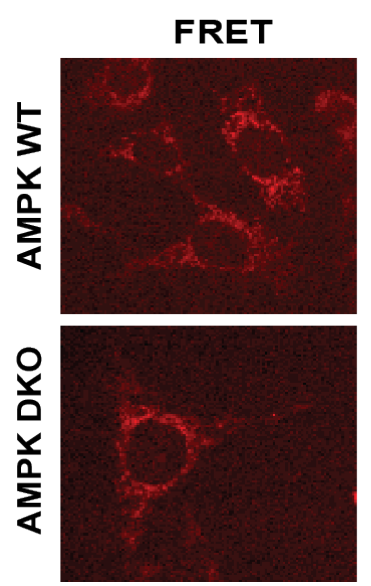

YFP
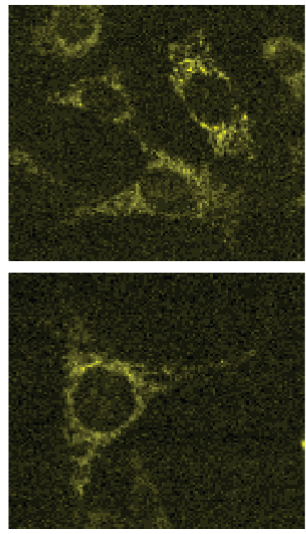

b

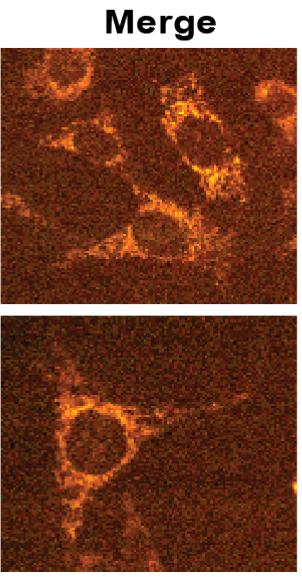

Basal Calcium

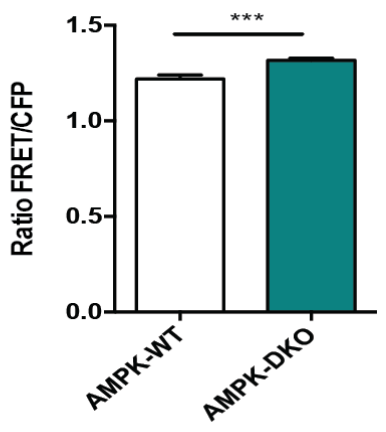

Figure 16 | Mitochondrial calcium is enhanced in AMPK DKO MEFs. (a) Representative fluorescence images of AMPK DKO and AMPK WT MEFs transiently transfected with mitochondrial $\mathrm{Ca}^{2+}$ sensor 4mtD3cpV $48 \mathrm{~h}$ before the measurements. Basal mitochondrial $\mathrm{Ca}^{2+}$ levels were recorded in Ringer's solution containing $0.5 \mathrm{mM} \mathrm{Ca}^{2+}$. (b) Quantification of basal mitochondrial $\mathrm{Ca}^{2+}$ in AMPK DKO (140 cells) and AMPK WT (138 cells). Graph represents mean \pm SEM of three independent experiments and t-test was performed. SEM: standard error of the mean. 


\subsection{AMPK mediates repression of MERCs via MFF}

\subsubsection{MFF is an AMPK target and a mitochondria-ER protein}

Given the observed increase in the number of contact sites in the absence of AMPK activity, we sought to understand which AMPK targets were in physical proximity of MERCs or played a role in their regulation. For this purpose, we crossed a published proteome of MERCs (Hung et al., 2017) with a list of established AMPK targets (Egan et al., 2011a; Gwinn et al., 2008). We found the MFF as the only protein, which scored both as an AMPK target and was localized to the MERCs (Figure 17a). This protein is known to be required for mitochondrial fission (Losón et al., 2013; Otera et al., 2010; Toyama et al., 2016). In order to study the cellular localization of MFF, subcellular fractionation experiments of WT and AMPK-DKO MEF were performed. Accordingly, MFF is present in 'crude' mitochondrial fractions, however the amount of MFF associated with the 'pure mitochondrial' fraction was robustly reduced in the AMPK-DKO cells while increased in the cytoplasm (Figure 17b). Additionally, in AMPK-DKO we can appreciate an increase of protein levels of MFN2 and TOM20 in ER fraction, which is in agreement with the increase of MERCs observed by EM. As markers for the different fractions, we used TOM20 and VDAC1 for the mitochondrial fraction, sec61 beta (ER protein) for the microsomal fraction and finally HPRT for the cytosolic fraction (Figure 17b). Taken together, this result suggests that localization of MFF in mitochondria is highly regulated by its phosphorylation by AMPK, consistent with the role of AMPK in activating mitochondrial fission (Toyama et al., 2016).

a

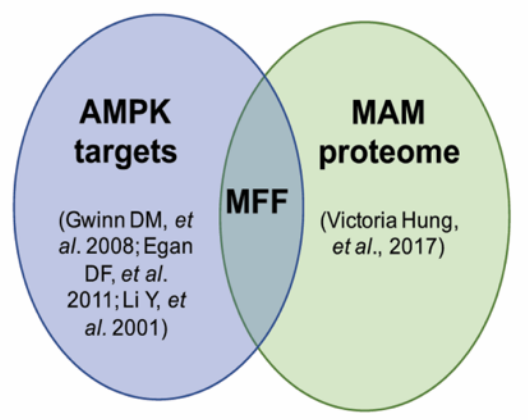

b

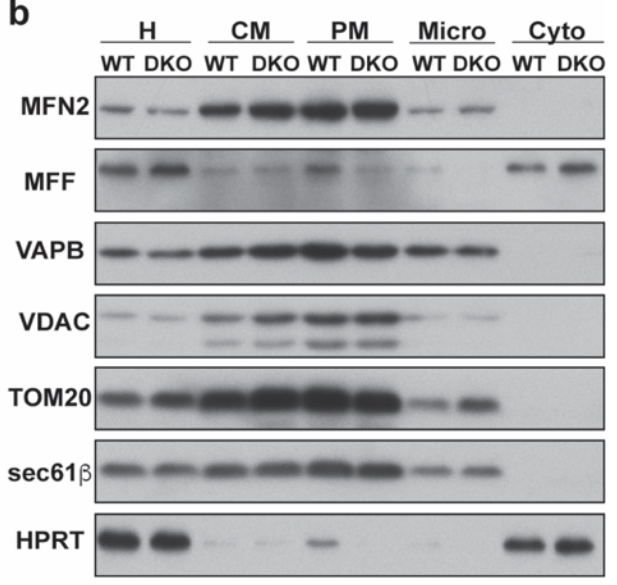

Figure 17 | MFF is a MERC-localized AMPK target. (a) Venn diagram showing overlap of AMPK targets with MAM proteome. (b) Immunoblots of subcellular fractions of AMPK MEFs subjected to gradient centrifugation. VDAC1 and TOM2O were used as markers of mitochondria, whereas sec61 beta for ER marker. $\mathrm{H}$, homogenate; $\mathrm{CM}$, crude mitochondria; PM, pure mitochondria; micro, microsomes; cyto, cytosol. 


\subsubsection{Increased number of MERCs in MFF KO cells}

As mentioned above, MFF was the only protein that scored as being involved in MERCs, as well as being an AMPK target. Therefore, to test if MFF is indeed involved in MERCs, we took advantage of mouse embryonic fibroblasts lacking MFF (MFF KO) to study MERCs (Liu and Chan, 2015). Electron microscopy of these cells was performed, and we observed a robust increase in the number of MERCs in MFF KO cells (Figure 18a and 18b). Notably, this was not a consequence of mitochondrial perimeter changes, since the average perimeter of mitochondria involved in MERCS was not different between WT and MFF KO cells (Figure 18c). However, the mitochondria not involved in contacts with the ER were significantly bigger in MFF KO, consistent with its role in mitochondrial fission.

a
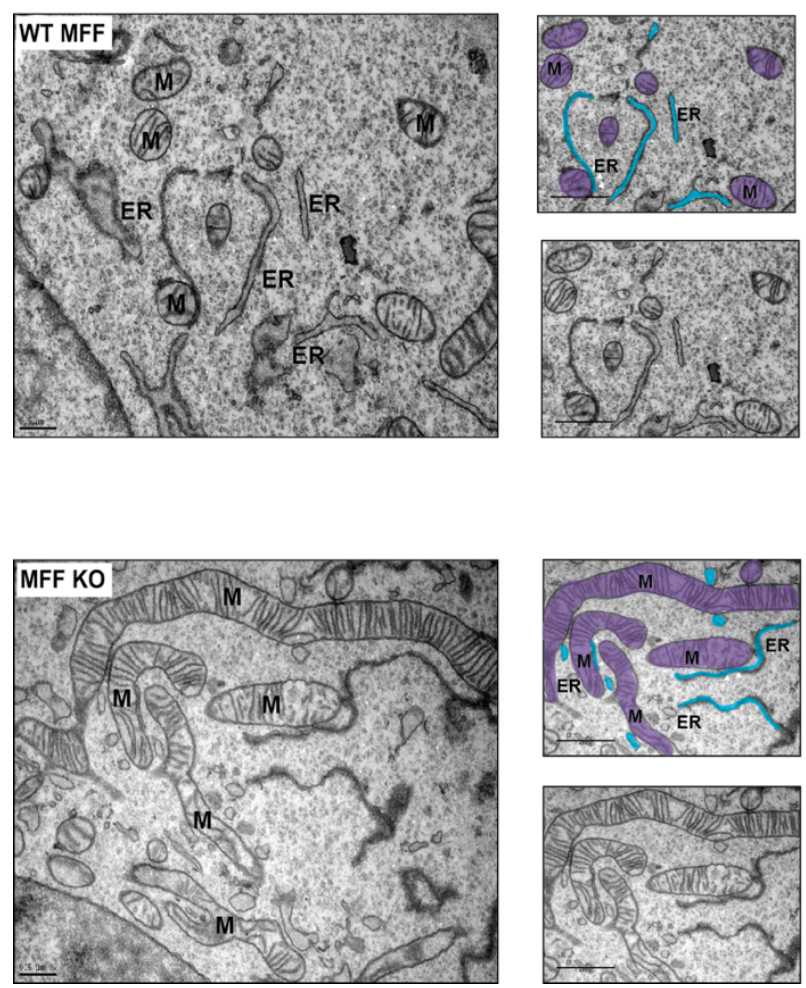

b

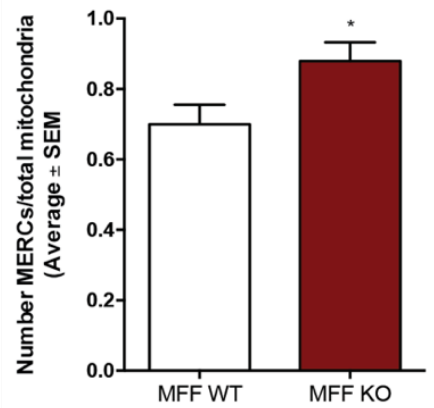

C

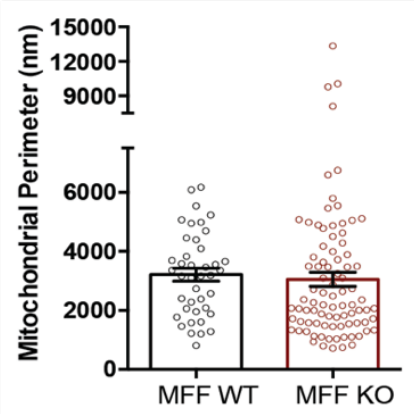

Figure 18 | Increased number of ER-mitochondria contact sites in MFF-KO model. (a) Representative EM images of MEFs of the indicated genotype (Scale bars, $0.5 \mu \mathrm{m}$ ) (b) Quantification of the number of ER-mitochondria contact sites in which the organelles are separated by less than 30-nm. Data represent mean \pm SEM of two independent experiments ( $\mathrm{n}>300$ mitochondria per experiment). (c) Morphometric analysis of mitochondrial perimeter involved in contact sites. Data represent mean \pm SEM of two independent experiments ( $n>300$ mitochondria per experiment). The differences were significant based on Mann-Whitney test. *P $<0.05$; SEM: standard error of the mean. 
Furthermore, the average length of the MERCs was similar in MFF KO and WT (Figure 19a), although longer contact sites were mostly observed in MFF KO, similar to the observation in AMPK-DKO cells (Figure 19b).

a

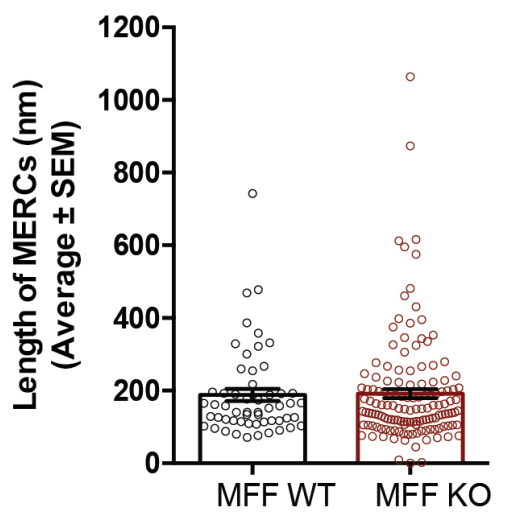

b

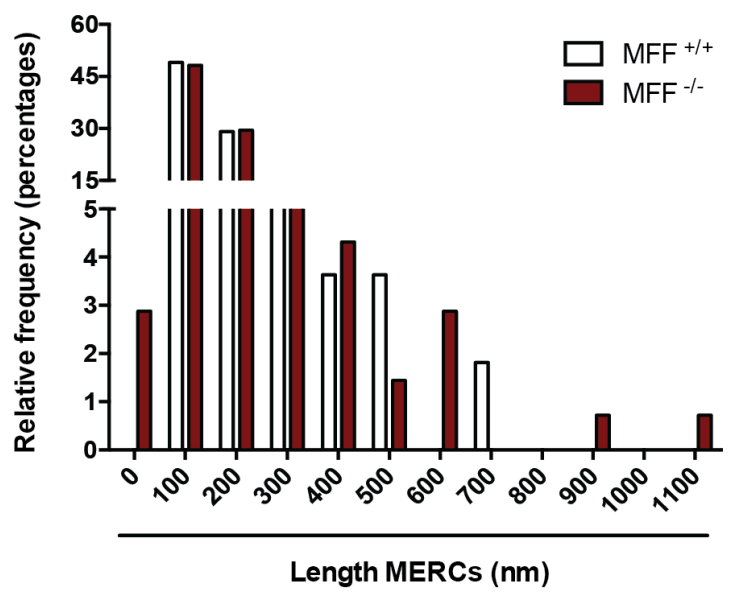

Figure 19 | MFF KO cells have longer contact sites than WT MFF cells. (a) Morphometric analysis of ER length located at less than 30-nm from mitochondria. Data represent mean \pm SEM of two independent experiments ( $\mathrm{n}$ $>300$ mitochondria per experiment) (b) Histogram reporting lengths quantified in (a). SEM: standard error of the mean.

\subsection{AMPK-dependent phosphorylation of MFF regulates MERCs}

\subsubsection{Phosphomimetic MFF Ser ${ }^{155}-S^{172}$ in AMPK-DKO cells has a gain of function of MFF}

The obtained results for MFF KO MEFs prompted us to further investigate the role of MFF on MERCs. For this reason, we tested if expression of a constitutively active isoform of MFF could normalize the increase in MERCs observed in AMPK-DKO MEFs. The outer mitochondria membrane protein, MFF, is a DRP1 receptor that triggers mitochondrial fission (Toyama et al., 2016). In addition, it is known that human MFF has two predicted AMPK phosphorylation sites, $\mathrm{Ser}^{155}$ and $\operatorname{Ser}^{172}$ (Figure 20a). In order to investigate the role of the AMPK-MFF axis in the context of MERCs, we applied the strategy described by Toyama and colleagues, by expressing WT MFF, a non-phosphorylatable Ser155Ala-Ser172Ala (SA2) mutant MFF, as well as, a phospho-mimetic Ser155Asp-Ser172Asp mutant (SD2) (Toyama et al., 2016) in AMPK-DKO cells. To prove this genetic approach, we took advantage of the fact that MFF is the major receptor for DRP1, and 
is required for mitochondrial DRP1 localization for subsequent mitochondria fission (Losón et al., 2013; Otera et al., 2010; Shen et al., 2014; Toyama et al., 2016). Therefore, we verified the functional role of MFF overexpression in AMPK-DKO cells using different approaches. We first evaluated the phosphorylation of DRP1 S616, which is associated with mitochondrial fission (Ko et al., 2016; Liesa et al., 2009; Taguchi et al., 2007). By western blot, we observed equal levels of total MFF in all conditions tested (WT MFF, SA2 MFF and SD2 MFF). However, for SD2 MFF mutant, we observed an increase in pDRP1 S616 phosphorylation when compared with WT MFF expressing cells (Figure $\mathbf{2 0 b}$ and 20c).

a
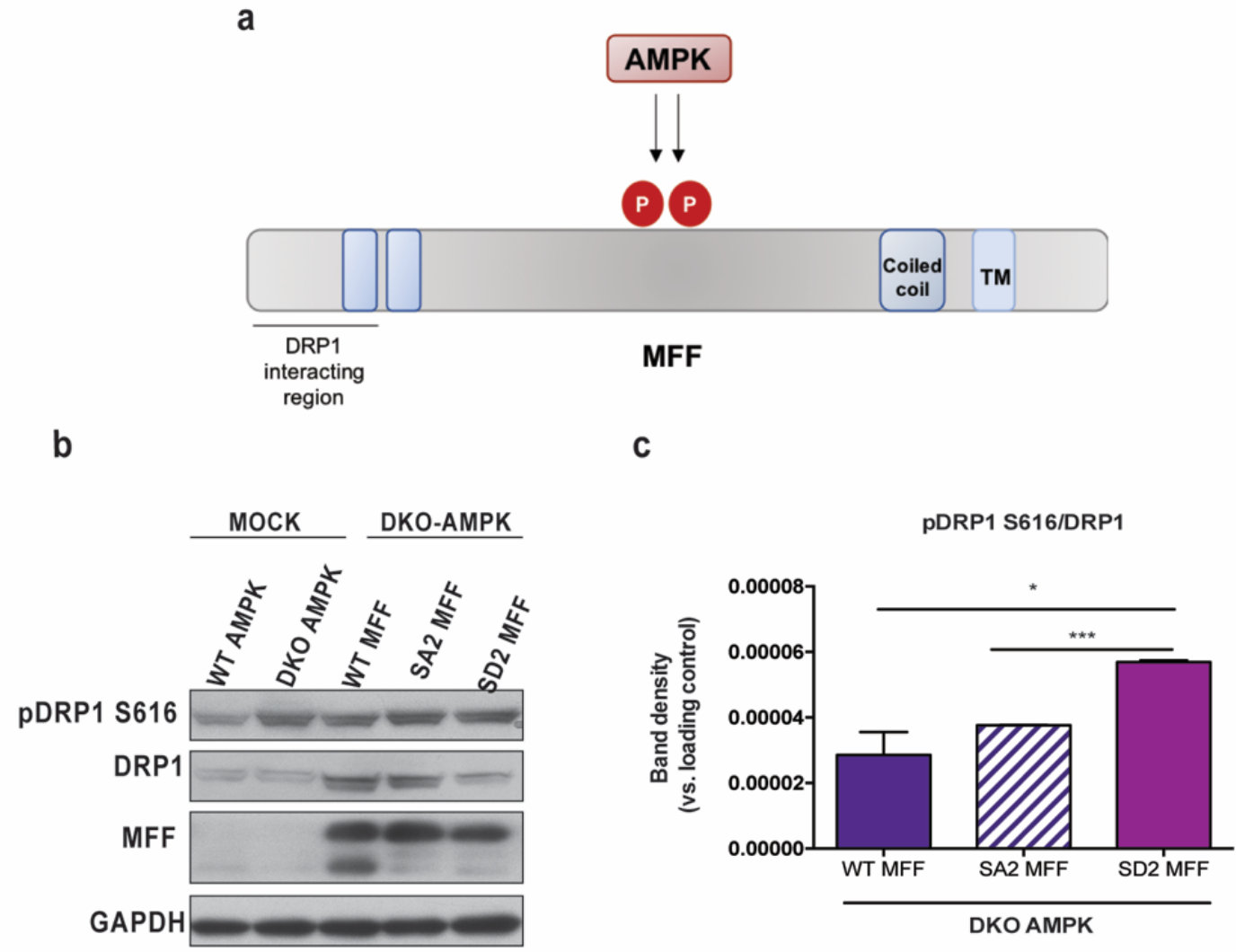

Figure 20 | Increase in pDRP1 S616 phosphorylation in SD2 MFF mutant. (a) Schematic representation of MFF illustrating location of Drp1-binding domains at amino-terminus and transmembrane domain which localizes MFF to outer mitochondrial membrane (b) Protein immunoblot of pDRP1 S616 phosphorylation in AMPK-DKO expressing MFF mutants (c) Quantification of immunoblots represented in (b). The differences were significant based on the t-test, ${ }^{*} \mathrm{P}<0.05 ;{ }^{* * *} \mathrm{P}<0.001$; $\mathrm{SEM}$ : standard error of the mean. 
Furthermore, we observed a gain-of-function activity in SD2 MFF mutant in AMPK-DKO MEFs, with cells displaying alterations in mitochondrial morphology, such as, shortened mitochondria, comparable with those expressing WT MFF (Figure 21a and 21b).

a

\section{DKO AMPK}

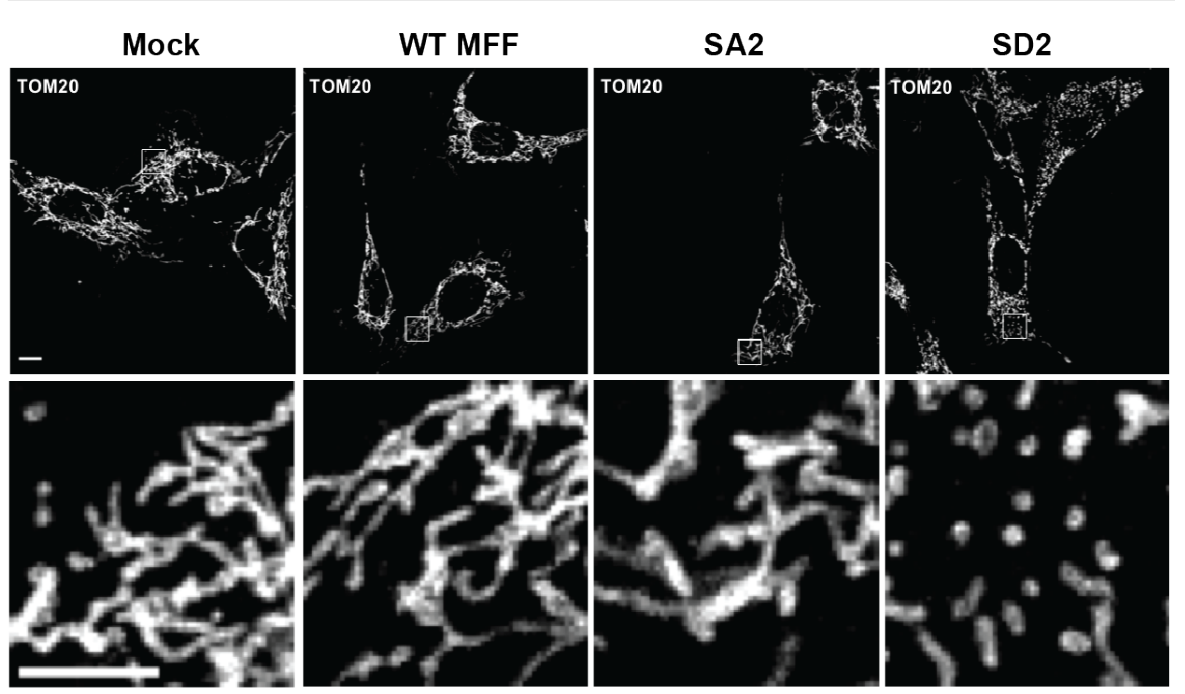

b

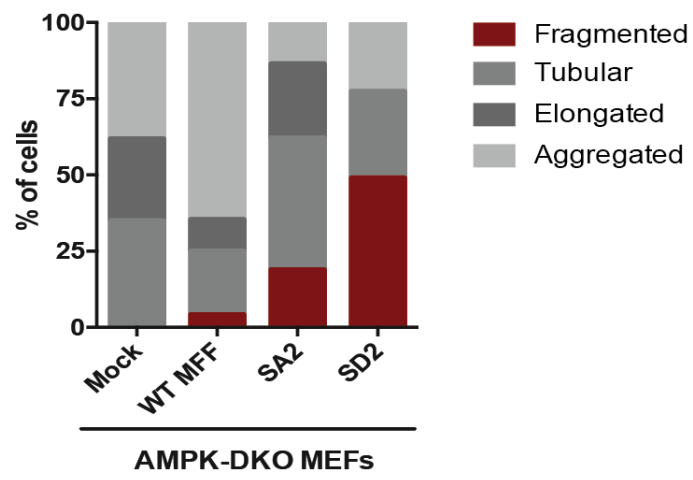

Figure 21 | Mitochondrial morphology of AMPK-DKO expressing different MFF mutants. (a) Representative immunostainings of TOM20 Mitochondrial morphology of AMPK-DKO + MFF mutants. Cells were stained for TOM20 (red) and analyzed by confocal imaging (Scale bar $10 \mu \mathrm{m}$ ). (b) Qualitative analysis of mitochondrial morphology of AMPK-DKO + MFF mutants. 


\subsubsection{MFF activity normalizes increased MERCs in AMPK-DKO cells}

Our next step was to examine the role of MFF in the remodeling of ER-mitochondria coupling. For this purpose, EM was performed on the AMPK-DKO MEFs stably expressing the MFF mutants described above, and several parameters to characterize MERCs and mitochondrial morphology were analyzed. Electron microscopy revealed that SD2 MFF mutant prevented the increase in contacts observed in AMPK-DKO MEFs (Figure 22b). Moreover, morphometric analysis showed a decrease in the average perimeter of mitochondria involved in MERCs in AMPK-DKOS with MFF expression when compared with WT AMPK cells (Figure 22c).

a
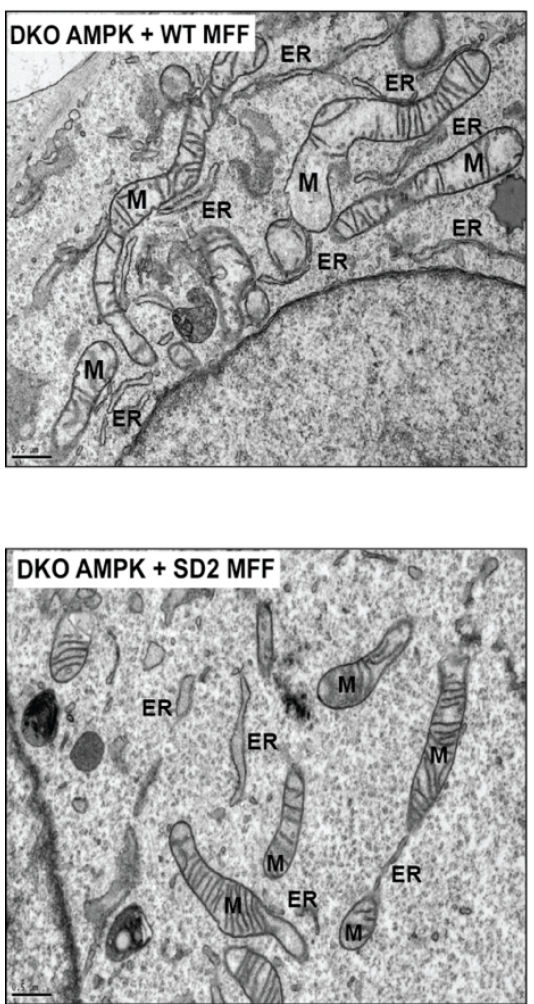

b
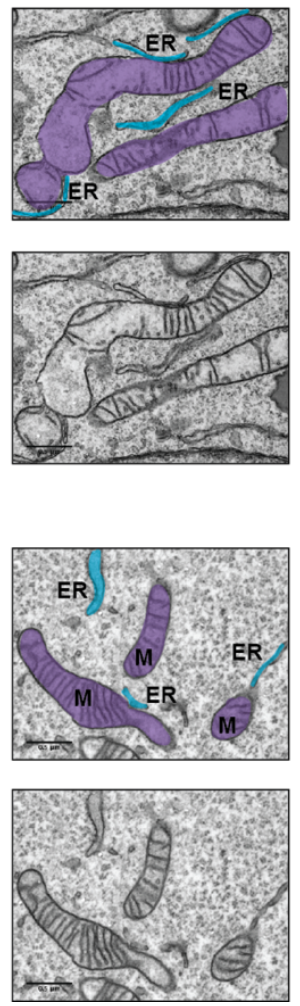

C
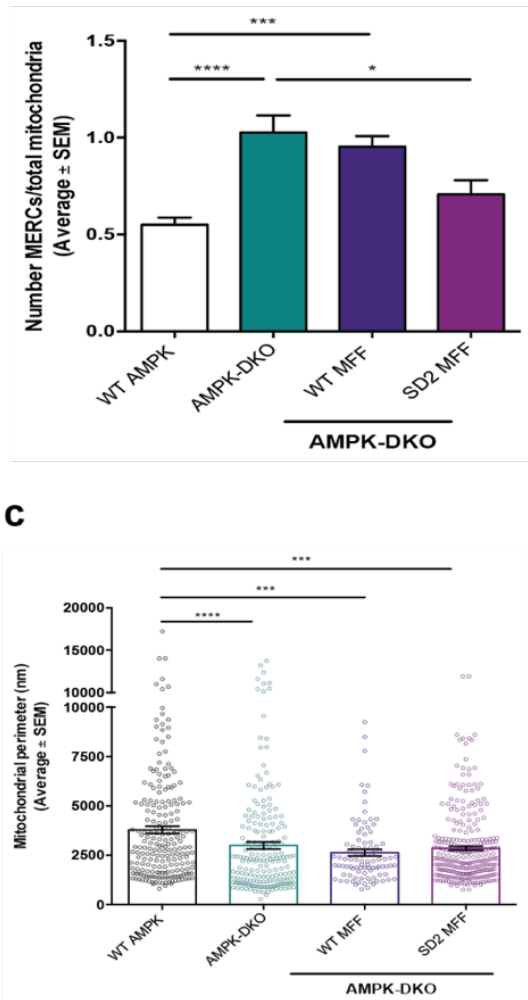

Figure 22 | AMPK regulates the number of ER-mitochondria contact sites via MFF. (a) Representative EM images of MEFs of the indicated genotype (Scale bars, $0.5 \mu \mathrm{m}$ ) (b) Quantification of the number of ER-mitochondria contact sites in which the organelles are separated by less than 30-nm. Data represent mean \pm SEM of two independent experiments ( $n>300$ mitochondria per experiment). (c) Morphometric analysis of mitochondrial perimeter involved in contact sites. Data represent mean \pm SEM of two independent experiments ( $n>300$ mitochondria per experiment). The differences were significant based on Mann-Whitney test. ${ }^{*} \mathrm{P}<0.05 ;{ }^{* *} \mathrm{P}<0.001 ;{ }^{* * * *} \mathrm{P}<0.0001 ;$ SEM: standard error of the mean. 
Regarding the extension of contact sites between both organelles, we observed an increase in length in cells expressing MFF mutants when compared with AMPK-DKO MEFs (Figure 23a and 23b). Furthermore, when comparing with WT AMPK cells, the AMPK-DKO cells expressing MFF mutants still displayed increased extension of MERCs, despite the reduced mitochondrial perimeter as described before (Figure 23a and 23b). Taken together, these results suggest that AMPK regulates the number of MERCs via MFF phosphorylation.

a

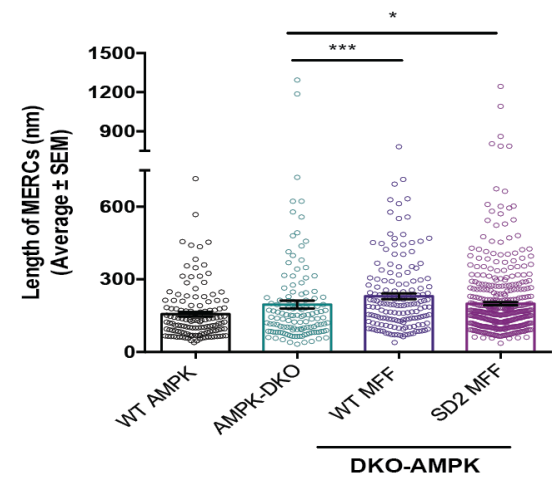

b

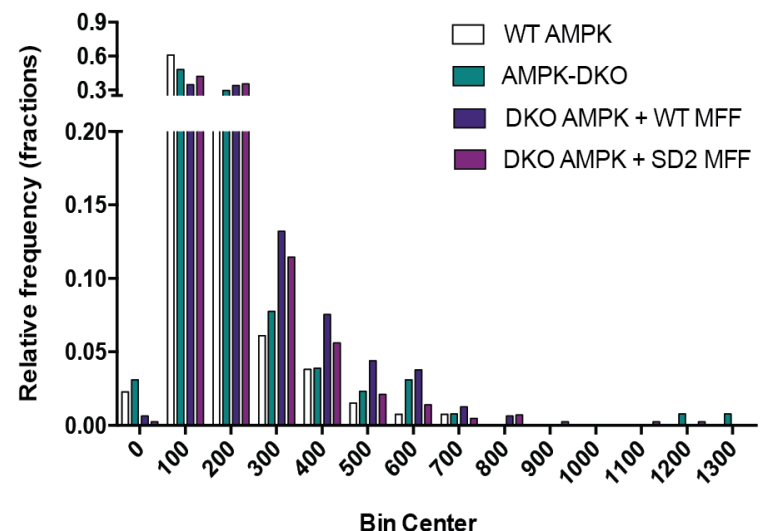

Figure 23 | Increased extension of ER-mitochondria contact sites in AMPK-DKO expressing MFF mutants. (a) Morphometric analysis of ER length located at less than 30-nm from mitochondria. Data represent mean \pm SEM of two independent experiments ( $\mathrm{n}>300$ mitochondria per experiment). (b) Histogram reporting lengths quantified in (a). SEM: standard error of the mean. The differences were significant based on Mann-Whitney test; $* P<0.05 ; * * * P<0.001 ;$ SEM: standard error of the mean.

\subsubsection{Mitochondrial function is improved with MFF genetic manipulation in} AMPK-DKO cells

Having demonstrated that MFF has a role in normalizing the increased number of MERCs in AMPK-DKO cells, we postulated that this rescue could also have an impact on mitochondrial respiration. As a result, oxygen consumption was measured in these cells using seahorse. We observed an enhanced OCR in AMPK-DKO expressing WT MFF and mutant SD2 MFF under basal conditions and upon inhibition or uncoupling of oxidative phosphorylation (maximal OCR) (Figure 24a-c). 
- AMPK-DKO - AMPK-DKO + WT MFF

- AMPKWT - AMPK-DKO +SD2 MFF

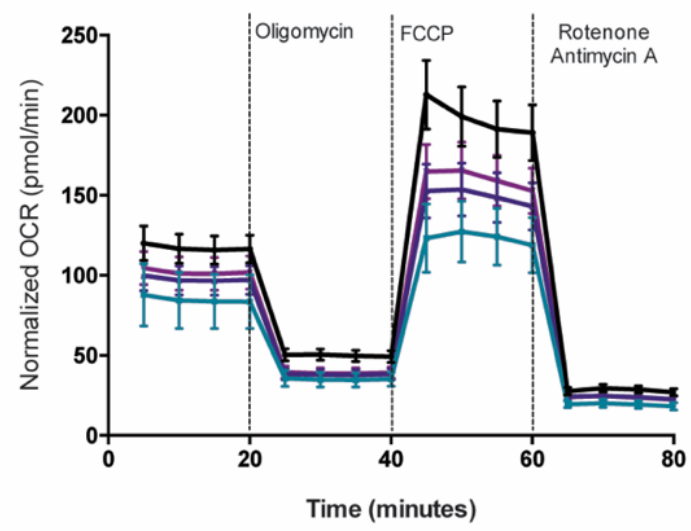

b

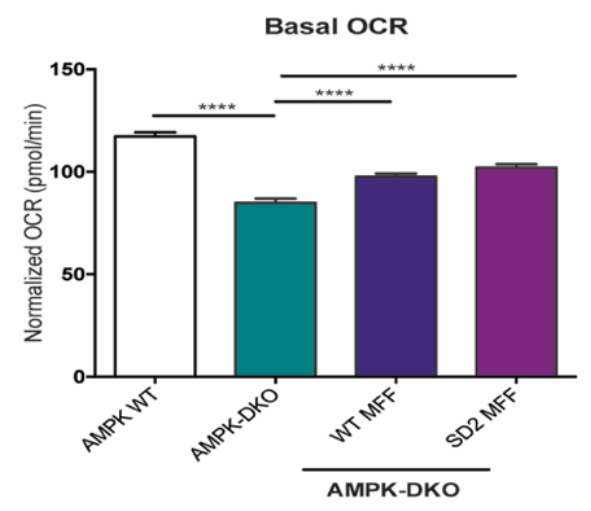

C

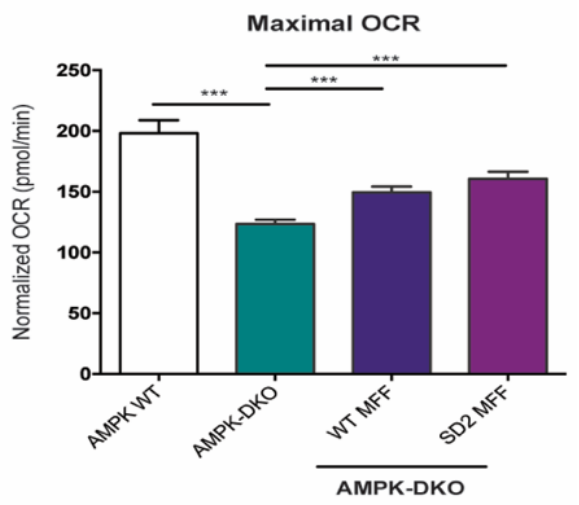

Figure 24 | Mitochondrial function is enhanced in AMPK-DKO expressing MFF mutants. (a) Mitochondrial OCR in WT, AMPK-DKO and in AMPK-DKO cells expressing mutant MFF (b) Increased basal mitochondrial OCR in AMPKDKO + MFF mutants MEFs as assessed by Real Time Respirometry (c) Increased maximal mitochondrial OCR in AMPK-DKO + MFF mutants. Graphs are mean \pm SEM for the indicated number of sample replicates and differences were significant based on the t-test, $* * * p<0.001 ; * * * * p \leq 0.0001 ;$ SEM: standard error of the mean.

\subsection{Activation of AMPK decreases mitochondria-ER contact sites}

\subsubsection{AMPK activation after acute mitochondrial stress decreases the number} of mitochondria-ER contact sites

Taking into account that absence of AMPK activity induces an increase in MERCs, we sought to test the opposite, of whether increasing AMPK activity results in a decrease of MERCs in WT MEF cells, as a bidirectional effect. Thus, we inhibited the mitochondrial respiratory chain, which we have previously shown to activate AMPK signaling due to the acute mitochondrial stress 
(Fernandez-Mosquera et al., 2019; Fernández-Mosquera et al., 2017). As such, WT MEFs were treated with antimycin A, an inhibitor of complex III of the mitochondrial respiratory chain, for 4 hours and AMPK activity was measured by assessing protein levels of AMPK (Figure 25a and 25b).

a

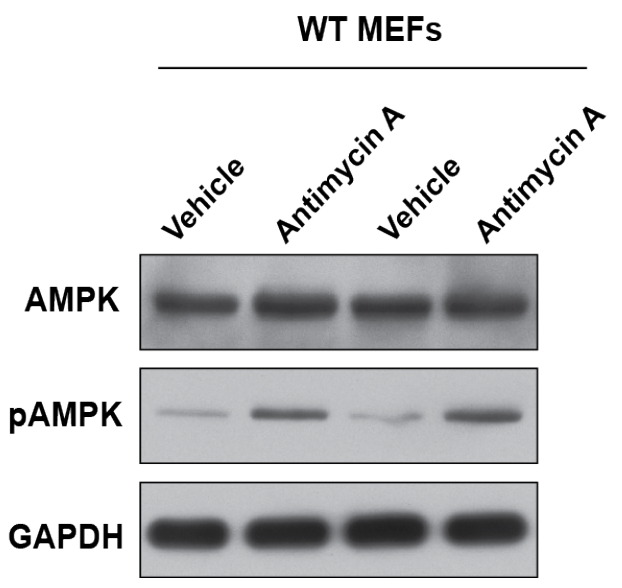

b

\section{pAMPKIAMPK}

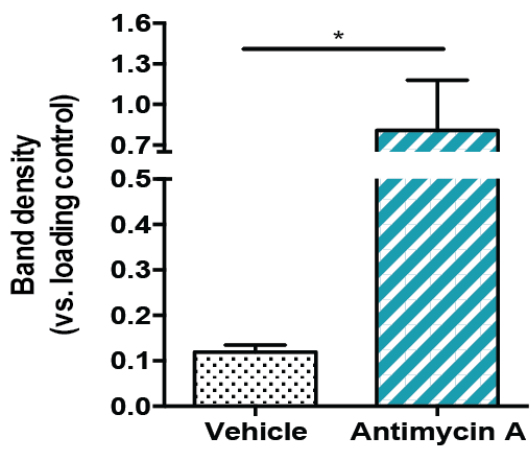

Figure 25 | Acute mitochondrial stress activates AMPK signaling. (a) Immunoblots showing the increase of AMPK phosphorylation. (b) Band density quantifications from the western blot represented in (a). Graphs are mean \pm SEM for the indicated number of sample replicates. The differences were significant based on the t-test, $* p<$ 0.05. SEM: standard error of the mean.

Morphometric analysis using electron microscopy was subsequently carried out, in order to get better insights into the effect of acute mitochondrial stress on mitochondria-ER remodeling. For this, antimycin A-treated cells were fixed and embedded. Based on analysis of electron micrographs (Figure 26a), we observed a striking decrease in the number of MERCs in antimycintreated cells (Figure 26b). It is noteworthy that this result was not a consequence of decreased mitochondrial perimeter since the average perimeter was increased in mitochondria involved in MERCs (Figure 26c). 
a
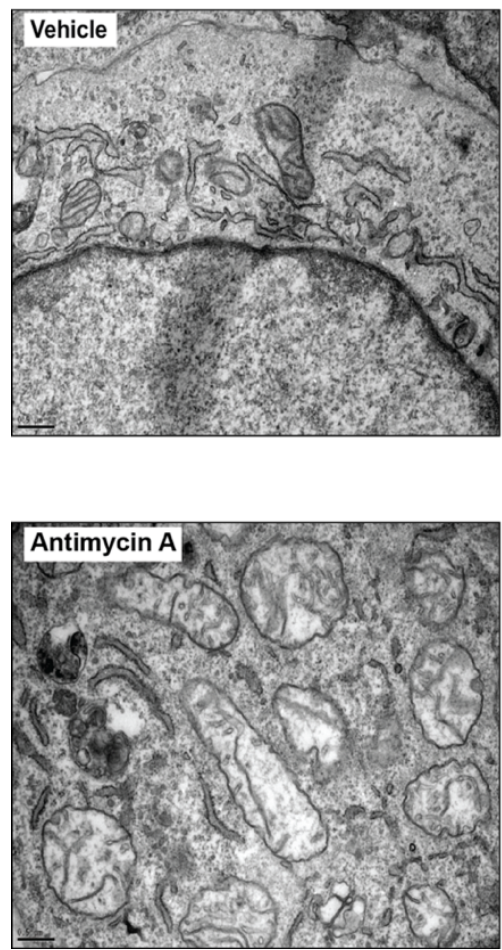
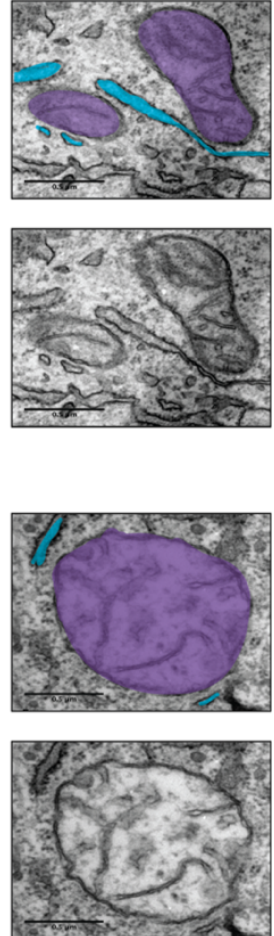

b

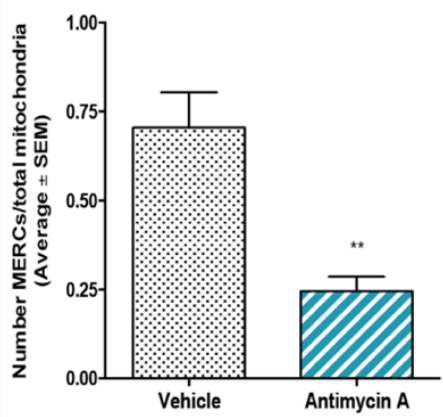

C

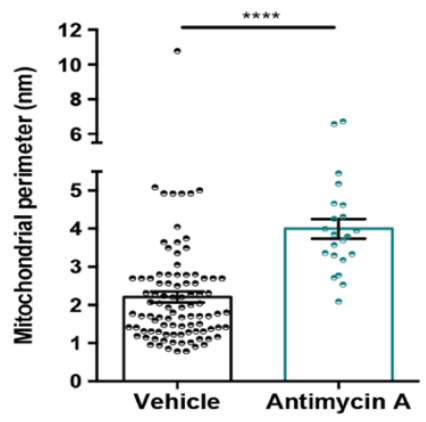

Figure 26 | Acute mitochondrial malfunction decreases the number of ER-mitochondria contact sites. (a) EM images of WT MEFs treated during $4 \mathrm{~h}$ with $10 \mu \mathrm{M}$ antimycin and ethanol as vehicle control (Scale bars, $0.5 \mu \mathrm{m}$ ) (b) Quantification of the number of ER-mitochondria contact sites in which the organelles are separated by less than 30-nm. Data represent mean \pm SEM of two independent experiments ( $n>300$ mitochondria per experiment). (c) Morphometric analysis of mitochondrial perimeter involved in contact sites. Data represent mean \pm SEM of two independent experiments ( $n>300$ mitochondria per experiment). The differences were significant based on Mann-Whitney test. ${ }^{* *} \mathrm{P}<0.01 ;{ }^{* * * * \mathrm{P}}<0.0001$ vs. Control; SEM: standard error of the mean.

Additionally, extension of ER involved in MERCs was quantified but no differences were obtained between control and antimycin A treatment (Figure 27a), despite an apparent shift towards longer contacts as observed by histogram analysis (Figure 27b). Altogether, these results show that acute mitochondrial insult, which induces AMPK activation, represses mitochondria-ER associations and alters mitochondrial morphology, as demonstrated by decreased number of MERCs and increased mitochondrial perimeter, respectively. Moreover, it confirms that AMPK has a bidirectional effect on mitochondria-ER remodeling. 


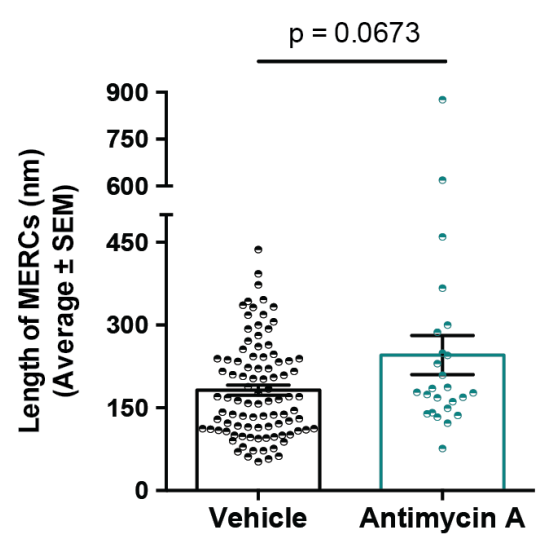

b

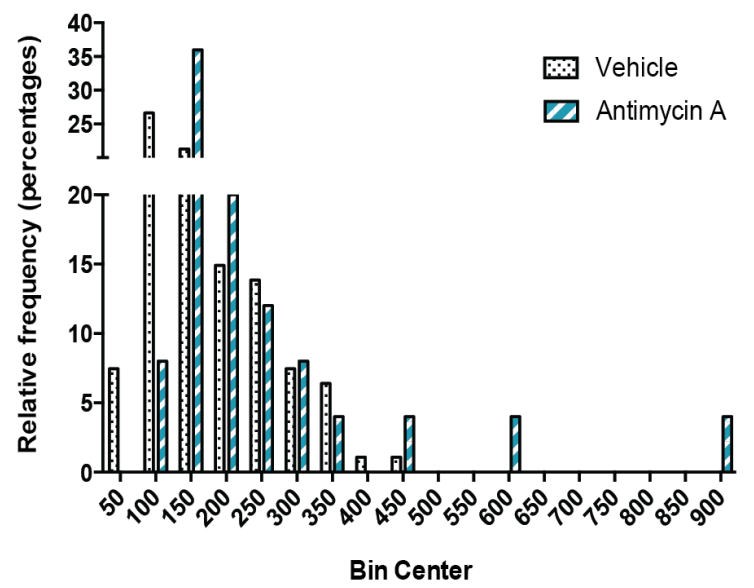

Figure 27| Increasing trend of MERCs extension in antimycin A treated cells (a) Morphometric analysis of ER length located at less than 30-nm from mitochondria. Data represent mean \pm SEM of two independent experiments ( $\mathrm{n}$ $>300$ mitochondria per experiment) (b) Histogram reporting lengths quantified in (a). The differences were significant based on Mann-Whitney test. ${ }^{* * *} \mathrm{P}<0.0001$ vs. Control; SEM: standard error of the mean.

\subsection{Chronic mitochondrial respiratory chain deficiency results in AMPK inhibition and leads to increased mitochondria-ER contact sites}

\subsubsection{AMPK activity is repressed in a model of chronic mitochondrial stress}

AMPK signaling is differentially regulated in acute and chronic mitochondrial stress (FernandezMosquera et al., 2019; Fernández-Mosquera et al., 2017). Therefore, we sought to understand if chronic impairment of the respiratory chain affects the associations between mitochondria and ER. For this purpose, we used HeLa cells with a lentivirus-delivered stable shRNA targeting the complex III subunit UQCRC1 (henceforth, RC-kd) and as control, we used HeLa cells with a lentivirus-delivered stable scrambled shRNA (henceforth, scrambled). Two different knockdowns of UQCRC1 (RC-kd A and RC-kd B) were prepared in order to evaluate the efficiency of each one. This mitochondrial malfunction model was previously characterized by FernandezMosquera et al, where several parameters associated with mitochondrial function were analyzed, including mitochondrial membrane potential, oxygen consumption rate and ROS production (Fernandez-Mosquera et al., 2019). This model presents lower oxygen consumption, decreased $\Delta \Psi \mathrm{m}$ and increased steady-state levels of reactive oxygen species (FernandezMosquera et al., 2019). Some of these parameters were again verified and reproduced, including the reduced protein levels of UQCRC1 in RC-kd cells and the lower OCR in basal and 
maximal respiration (Figure 28a-d). Furthermore, as previously described, our model of chronic mitochondrial malfunction has reduced AMPK activity (Fernandez-Mosquera et al., 2019). Interestingly, we also observed a reduced protein levels of MFF in RC-kd cells (Figure 28e).

a

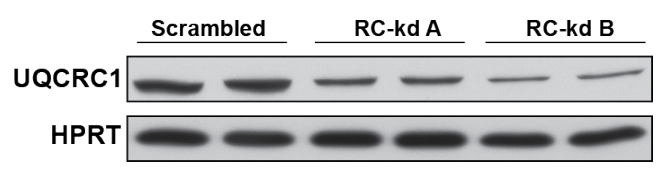

C

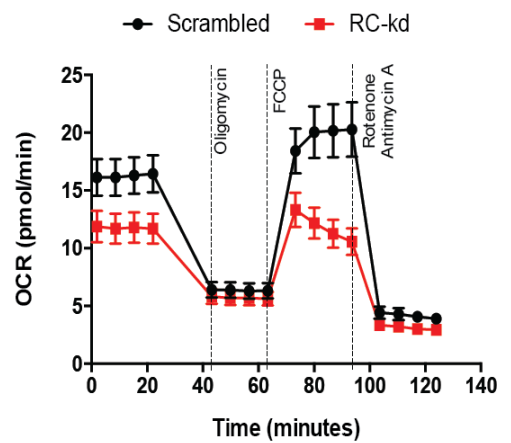

b

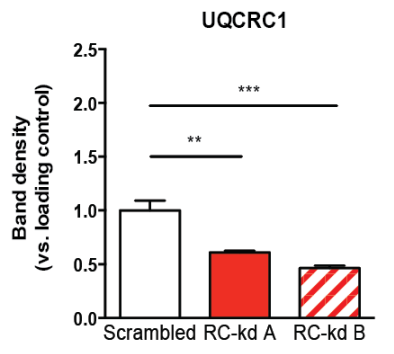

d

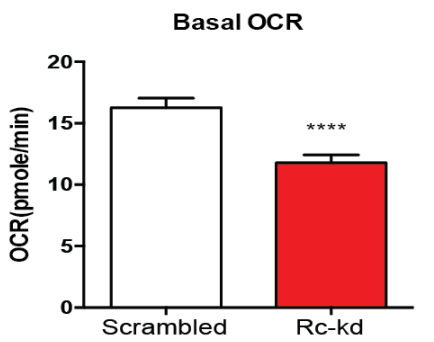

e

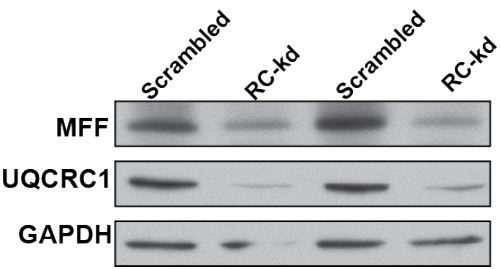

Figure 28 | Mitochondrial function is compromised in RC-kd HeLa cells. (a) Immunoblots showing the efficiency of a stable knock-down of UQCRC1. (b) Band density quantifications from the western blot represented in (a), (c) and (d) Decreased basal mitochondrial OCR in RC-kd cells as assessed by Real Time Respirometry. (e) Immunoblots from RC-kd cells and scrambled cells, showing a decrease of MFF protein level. Graphs are mean \pm SEM for the indicated number of sample replicates. The differences were significant based on the t-test, ${ }^{* *} p<0.01 ;{ }^{* * *} p$ $<0.001 ; * * * * p \leq 0.0001$. SEM: standard error of the mean. 


\subsubsection{Increased MERCs in chronic mitochondrial malfunction}

In order to investigate whether chronic mitochondrial malfunction has an impact on the MERCs, we prepared and obtained electron micrographs of RC-kd cells (Figure 29a). Based on the analysis of these micrographs, we observed an increased number of MERCs in RC-kd when compared with scrambled cells (Figure 29b). It is important to note that this increase was not a consequence of increased mitochondrial length, since the average perimeter of mitochondria involved in contact sites was decreased in RC-kd cells (Figure 29c).

a
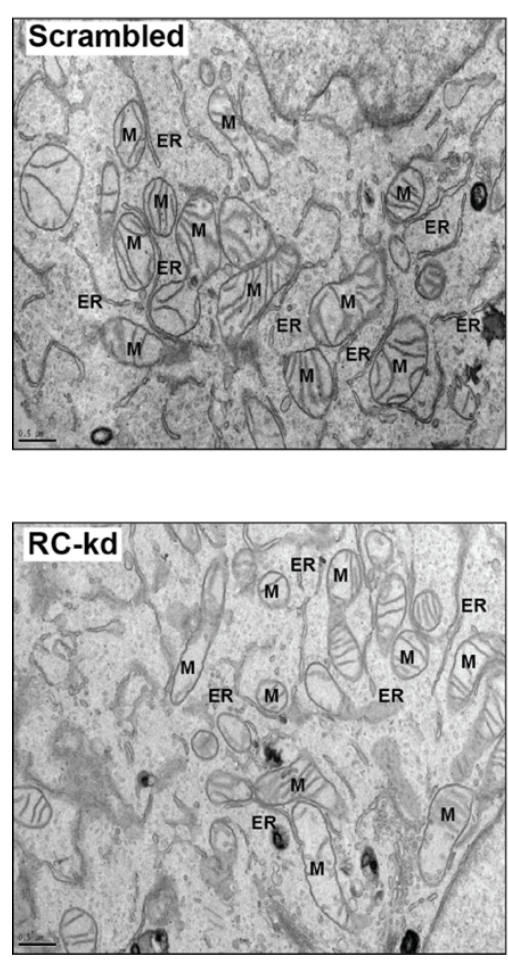
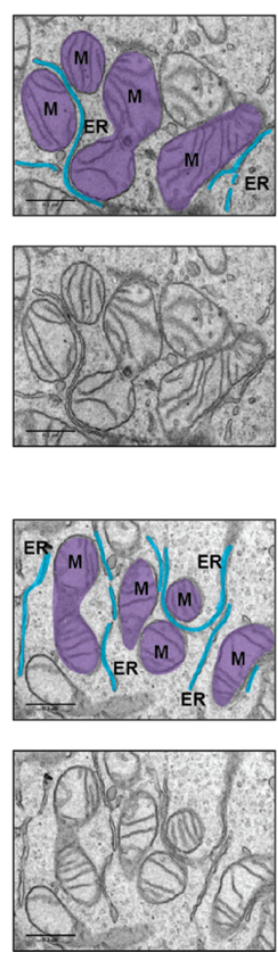

b

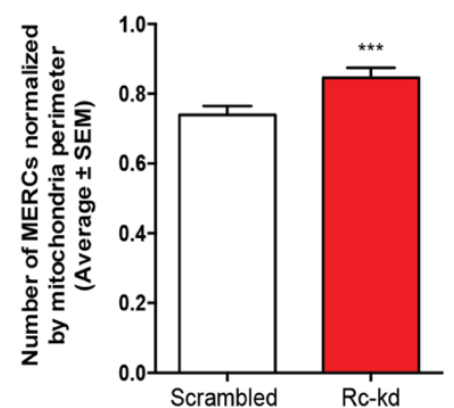

C

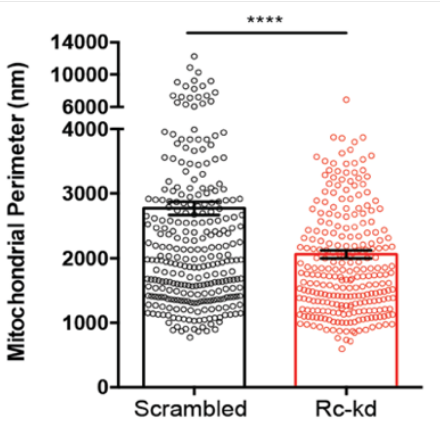

Figure 29 | Increased ER-mitochondria contact sites in chronic mitochondrial dysfunction. (a) Representative EM images of HeLa RC-kd as well as the corresponding control (scrambled) (Scale bars, $0.5 \mu \mathrm{m}$ ). (b) Quantification of the number of mitochondria-ER contact sites in which the organelles are separated by less than 30-nm. Data represent mean \pm SEM of two independent experiments ( $n>300$ mitochondria per experiment). (c) Morphometric analysis of mitochondrial perimeter involved in contact sites. Data represent mean \pm SEM of two independent experiments ( $n>300$ mitochondria per experiment). The differences were significant based on Mann-Whitney test, ${ }^{* * *} \mathrm{p}<0.001 ;{ }^{* * *} \mathrm{p} \leq 0.0001$. SEM: standard error of the mean.

Regarding the extension of the contact sites analyzed, an increase of the average length was observed in RC-kd cells when compared with scrambled cells (Figure 30a), as underscored by a 
slight increase in the length of individual contact sites (Figure 30b). Taken together, these data, supported by our previous findings, suggest that increased mitochondria-ER associations in RC$k d$ cells could be attributed to the repressed AMPK signaling in this model.

a

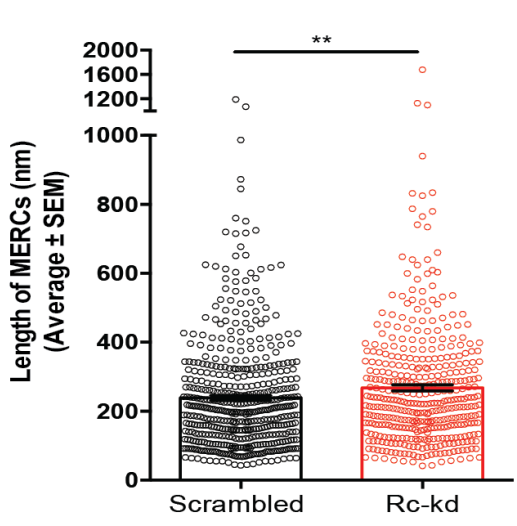

b

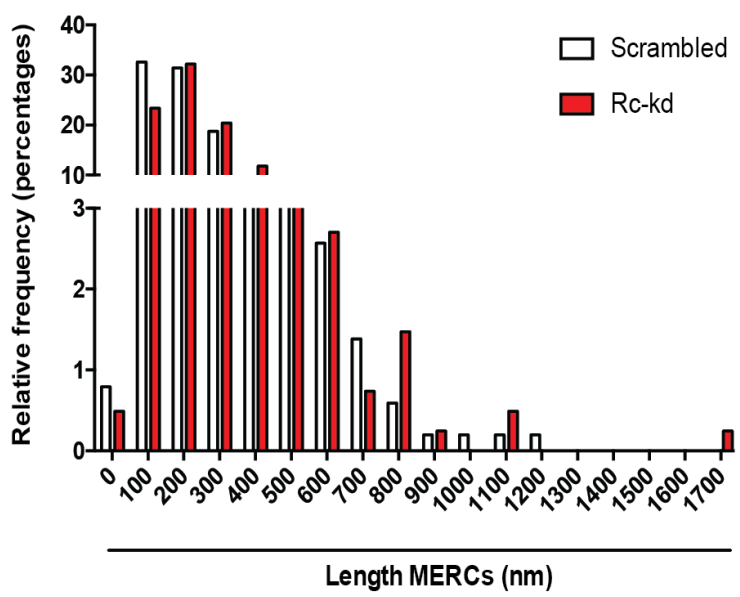

Figure 30 | Increased MERC extension in chronic mitochondrial dysfunction. (a) Morphometric analysis of ER length located at less than 30-nm from mitochondria. Data represent mean \pm SEM of two independent experiments ( $n>300$ mitochondria per experiment). (b) Histogram reporting lengths quantified in (a). Data represent mean \pm SEM of two independent experiments ( $n>300$ mitochondria per experiment). The differences were significant based on Mann-Whitney test, ${ }^{* *} \mathrm{P}<0.01$. SEM: standard error of the mean.

\subsubsection{Increased abundance of MERC related proteins in RC-kd}

Several protein complexes have been established for a long time to be involved in organelle appositions of ER and mitochondria. Some examples include MFN2, which is present both in the outer mitochondrial membrane and in the ER membranes, and its homodimers keep mitochondria tethered to the ER (De Brito and Scorrano, 2008). Moreover, another ER-mito-CS complex is formed by the PTPIP51 in the outer mitochondrial membrane and VAP-B in the ER. This particular contact site tether has been associated with the regulation of the extension of MERCs, since overexpression of either PTPIP51 or VAPB results in increased MERCs extension, and their knock-down causes a corresponding decrease (Stoica et al., 2014). Given the crucial role of these tethers in MERCs, we evaluated their whole cell protein expression in our model of chronic mitochondrial malfunction by western blot. As shown in Figure 31, we observed an increase of VAPB and MFN2 protein levels in RC-Kd cell model (Figure 31a and 31b). We observed no changes in the protein levels of PTPIP51 in RC-kd cells (Figure 31b). 
a

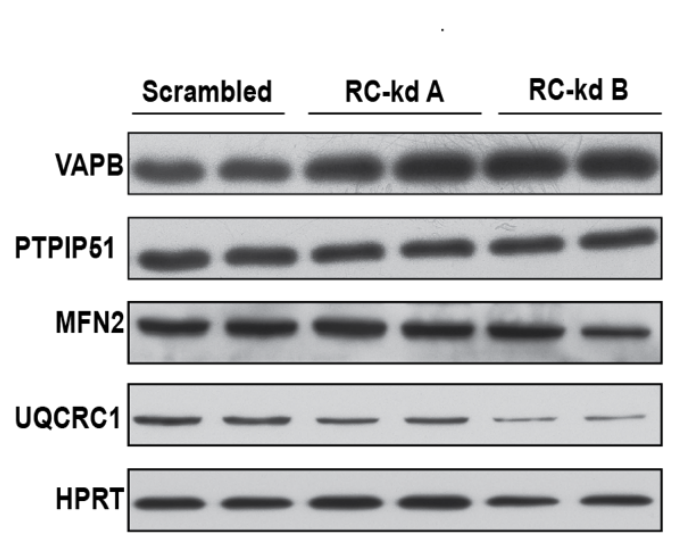

b

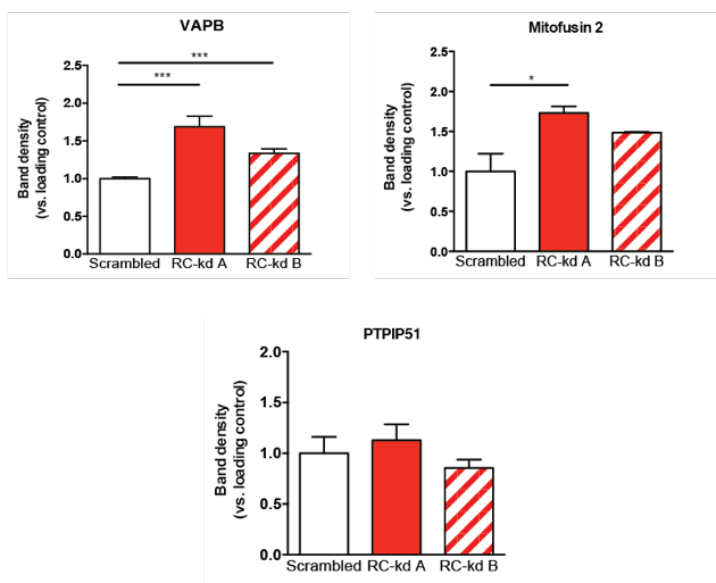

Figure 31 | Increased protein levels of tethers involved in mitochondria-ER associations in a RC-kd model. (a) Immunoblots of whole cell lysates from scrambled and RC-kd cells. HPRT was used as a loading control. (b) Band density quantification from the western blot represented in (a). Graphs are mean \pm SEM for at least sample triplicates and differences were significant based on the t-test, ${ }^{*} p<0.05 ;{ }^{* * *} p<0.001$. SEM: standard error of the mean.

In addition, since it is the complex between the two components of each ER-mitochondrial tethers that is relevant for the MERCs assembly/stability, we tested whether the interaction between VAPB and PTPIP51 was affected by immunoprecipitating endogenous VAPB and quantifying how much PTPIP51 was associated with it. From our results, we observed an increased interaction between both proteins in RC-kd when compared with scrambled cells (Figure 32a and 32b). Due to the fact that the immunoprecipitation was performed in whole cell extracts, it is possible that we are spuriously including PTPIP51-VAPB interaction that is not involved in MERCs. Notwithstanding, it is known that the PTPIP51-VAPB protein complex is typically located in MERCs (Stoica et al., 2014; De Vos et al., 2012), accordingly their interaction is directly correlated with MERCs. Therefore, this result supports an increased association of PTPIP51-VAPB, contributing to the increase of MERCs observed by EM. 
a

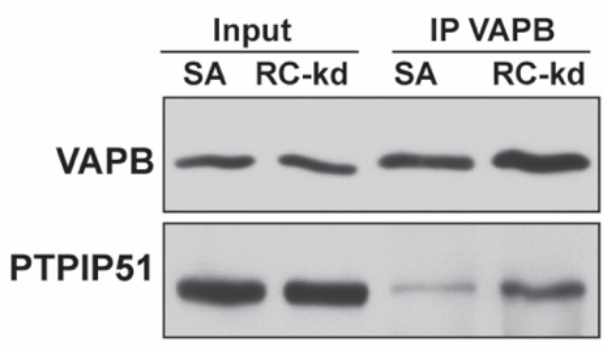

b

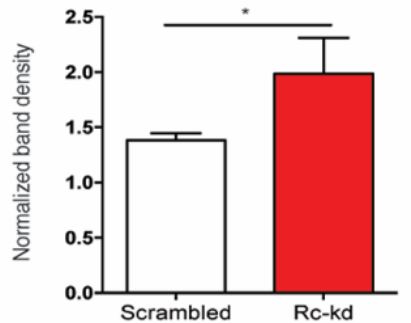

Figure 32 | Increased interaction of VAPB-PTPIP51 tether complex in RC-kd HeLa cells. (a) Immunoblots showing VAPB pull-down, as well as, PTPIP51 interaction in RC-kd and scrambled control. (b) Band density quantification from (a). Graphs are mean \pm SEM for at least six samples and differences were significant based on the t-test, $* p<0.05 ;$ SEM: standard error of the mean.

\subsubsection{Mitochondrial calcium $\left(\mathrm{Ca}^{2+}\right)$ uptake is enhanced in RC-kd cells}

Since we observed an enhancement of the number of contact sites in our model of mitochondrial malfunction, we hypothesized that this effect consequently could affect calcium trafficking mediated by MERCs. Thus, we employed the same strategy explained above to evaluate mitochondrial $\mathrm{Ca}^{2+}$ transfer. Scrambled and RC-kd cells were transfected with 4 mtD3cpv (mitochondrial cameleon) and followed by live imaging, during which they were treated with histamine, a stimulator of IP3Rs, resulting in a release of $\mathrm{Ca}^{2+}$ from ER and consequently, increasing mitochondrial $\mathrm{Ca}^{2+}$ uptake at the MERCs.

The basal levels of $\mathrm{Ca}^{2+}$ measured in mitochondria seemed higher in the RC-kd cells when compared with scrambled, which is consistent with the increased number of MERCs observed previously (Figure 33a). Since we observed a repression in AMPK signaling in RC-kd, we sought to investigate how mitochondrial $\mathrm{Ca}^{2+}$ levels can be modulated by activation of AMPK in RC-kd cells. Interestingly, A769662 treatment in RC-kd cells resulted in reduced basal levels of mitochondrial $\mathrm{Ca}^{2+}$ when compared to RC-kd cells without treatment (Figure 33a). Furthermore, scrambled cells showed a peak of mitochondrial $\mathrm{Ca}^{2+}$ after histamine treatment but RC-kd were almost non-responsive to histamine, showing only a residual increase in mitochondrial $\mathrm{Ca}^{2+}$ (Figure 33b). This non-responsiveness can be explained by $\mathrm{Ca}^{2+}$ saturation in the RC-kd cells, although this premise requires further testing. However, when we activate AMPK signaling in RC-kd during 4 hours of treatment with AMPK activator, A769662, we observed a normalization of mitochondrial $\mathrm{Ca}^{2+}$ uptake in response to histamine (Figure 33b). 
a

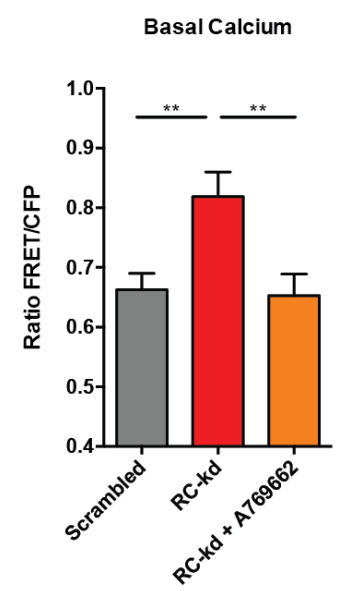

b

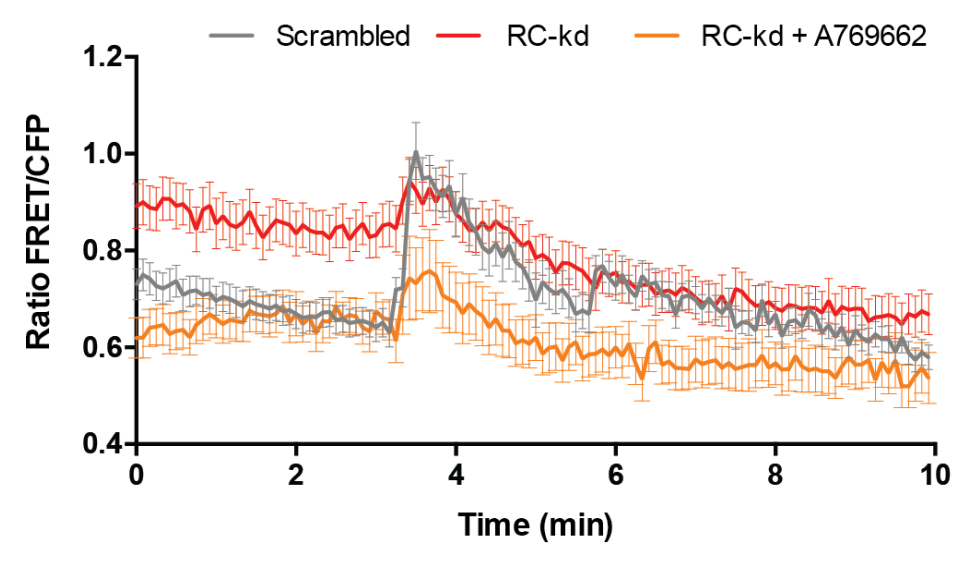

Figure 33 | Mitochondrial Ca ${ }^{2+}$ uptake is enhanced in RC-kd cell model. (a) Quantification of basal mitochondrial $\mathrm{Ca}^{2+}$. Data from at least six independent experiments ( $\mathrm{n} \geq 10$ recordings per experiment). (b) Mean \pm SEM of calculated FRET ratio in Ringer's buffer containing $0.5 \mathrm{mM}$ of $\mathrm{Ca}^{2+}$ where HeLa RC-kd and Scrambled were transiently transfected with mitochondrial $\mathrm{Ca}^{2+}$ sensor $4 \mathrm{mtD} 3 \mathrm{cpV}$ before the measurements. Cells were also treated with $100 \mu \mathrm{m}$ A769662 or DMSO (as control) during 4h. ER calcium release was stimulated with $1 \mathrm{mM}$ of histamine at 40 cycles of measurement. The differences were significant based on the t-test, ${ }^{*} \mathrm{p}<0.05 ; * *<0.01$; $* * * * \mathrm{P}<0.0001 . \mathrm{SEM}$ : standard error of the mean.

\subsubsection{Mitochondrial function is compromised in NDUFS4 $K O$ cells}

In order to support our findings in the chronic mitochondrial model, we also used another cellular model with permanent mitochondrial malfunction, NDUFS4 $K O$. These cells are embryonic fibroblasts derived from mice lacking mitochondrial complex I subunit NDUFS4. The mitochondrial function in this model was characterized by evaluating mitochondrial respiration, ROS production and mitochondrial membrane potential. Mitochondrial membrane potential was measured by flow cytometry using JC-1 dye as described above (Figure 34a). As mentioned before, for this experiment different subpopulations were obtained accordingly with the different intensities in both green and red channels for the JC-1 dye. From our results, we observed $10.2 \%$ of NDUFS4 KO and only $6.72 \%$ of WT cells in the depolarized population (Q3), suggesting an increase in the loss of $\triangle \Psi \mathrm{m}$ in NDUFS4 KO cells (Figure 34a and b). Furthermore, mitochondrial ROS production was also evaluated using MitoSOX probe. We observed an increase in the steady state levels of mitochondrial superoxide anion in NDUFS4 KO cells (Figure 34c). Regarding oxygen consumption rate, a robust decrease in basal OCR was observed in the 
NDUFS4 KO cells. Altogether, as expected, these data support the mitochondrial impairment in this model of mitochondrial malfunction.

a

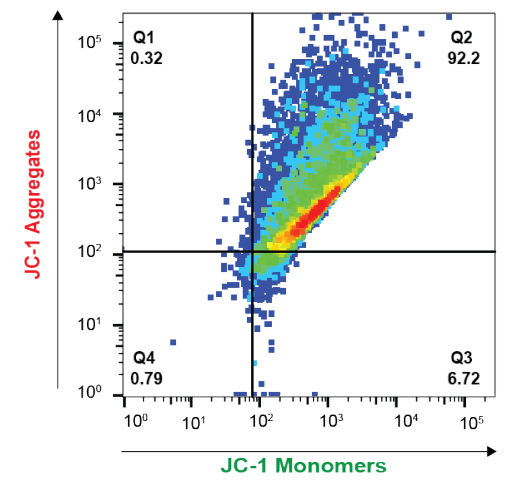

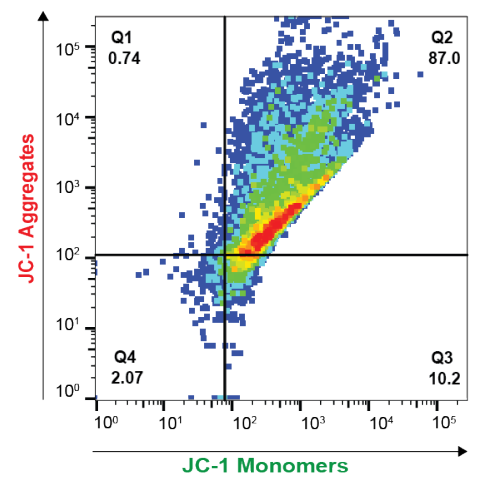

b

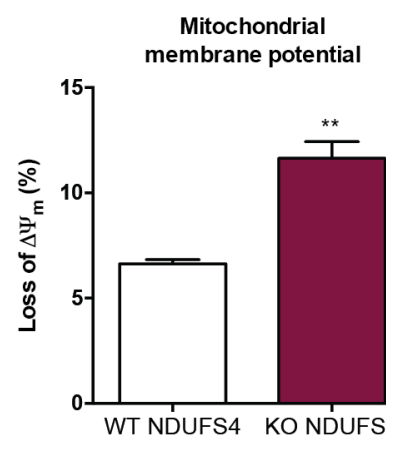

C

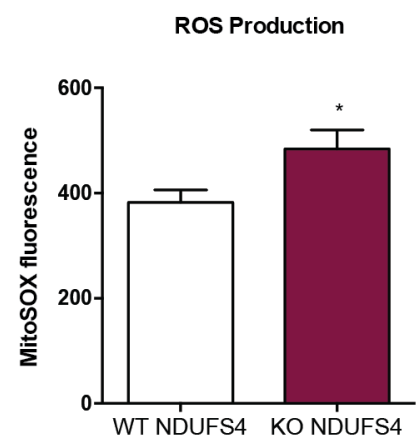

d

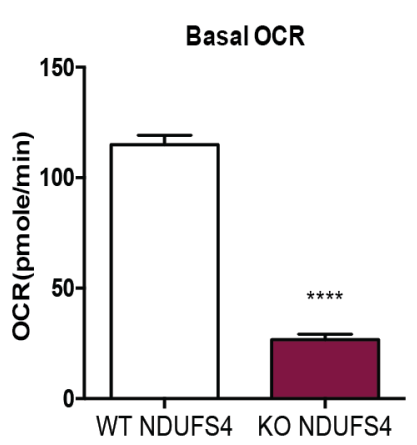

Figure 34 | Mitochondrial function is compromised in NDUFS4 KO model. (a) JC1-monomers vs. JC1-aggregates plot for NDUFS4 WT and KO cells with JC-1 dye. (b) Percentage of WT and KO cells with only green positive signal, represented as Q3 subpopulation. Data are representative for three independent measurements. (c) Increased MitoSox fluorescence in KO cells, which reflects mitochondrial superoxide levels. (d) Decreased basal mitochondrial OCR in NDUFS4 KO as assessed by Real Time Respirometry. Graph represents mean \pm SEM for the indicated number of sample replicates. The differences were significant based on the t-test, ${ }^{*} p<0.05 ;{ }^{* *} \mathrm{p}<0.01$; $* * * * P<0.0001$. SEM: standard error of the mean.

\subsubsection{Increased MERCs in NDUFS4 KO cell model}

Having established that a chronic model of mitochondrial malfunction (RC-kd cells) has an increased number of MERCs relative to the control cells, we decided to test another model of chronic mitochondrial malfunction with respect to MERCs using the same parameters as described before. We carried out electron microscopy of NDUFS4 WT and NDUFS4 KO cells for 
morphometric analysis (Figure 35a). We observed an increasing trend in the number of MERCs (Figure 35b) and in the perimeter of the mitochondria involved in contact sites with ER (Figure 35c) in NDUFS4 KO cells.

a
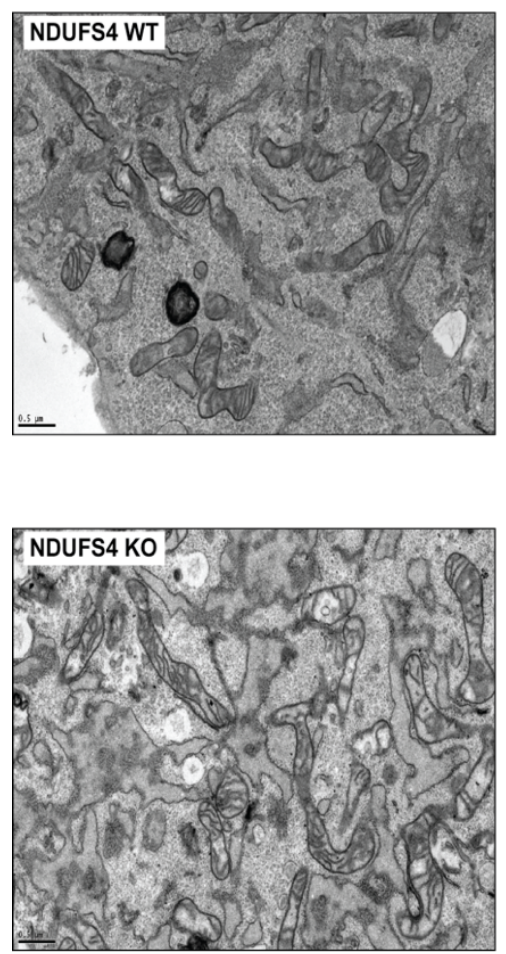
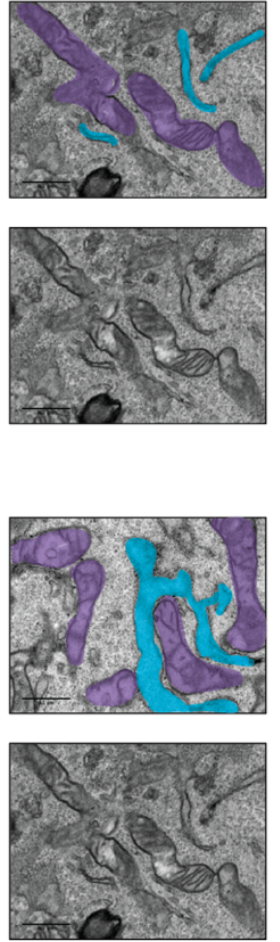

b

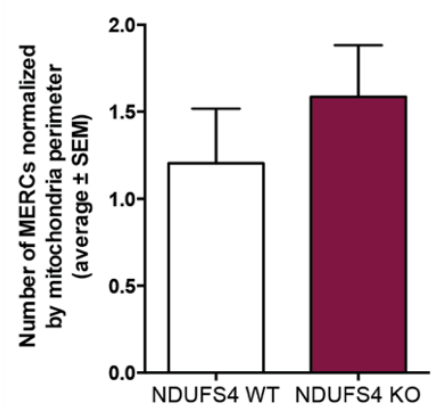

C

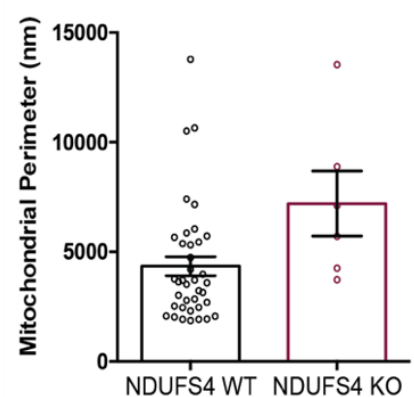

Figure 35 | Increasing trend of ER-mitochondria contact sites in NDUFS4 KO cell model. (a) Representative EM images of NDUSF4 KO as well as the corresponding control (Scale bars, $0.5 \mu \mathrm{m}$ ). (b) Quantification of the number of ER-mitochondria contact sites located at 30-nm maximum distance from outer mitochondrial membrane. (c) Morphometric analysis of mitochondrial perimeter involved in contact sites.

Taking into consideration the extension of ER in contact with mitochondria, we observed a significant reduction of the MERCs length in NDUFS4 KO cells when compared with WT cells (Figure 36a). Similarly, the distribution of the length reflected shorter extensions in NDUFS4 KO cells relative to WT cells (Figure $\mathbf{3 6 b}$ ). 
a

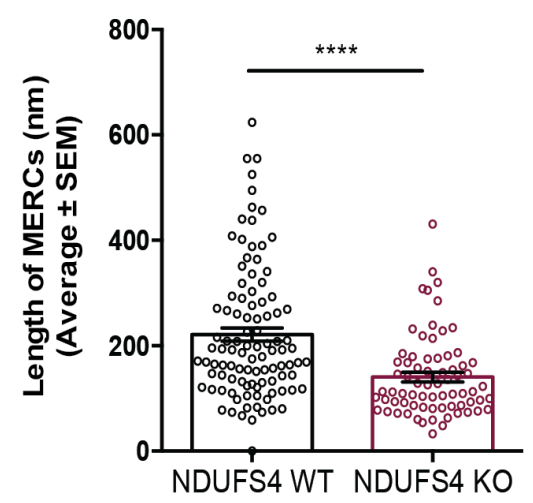

b

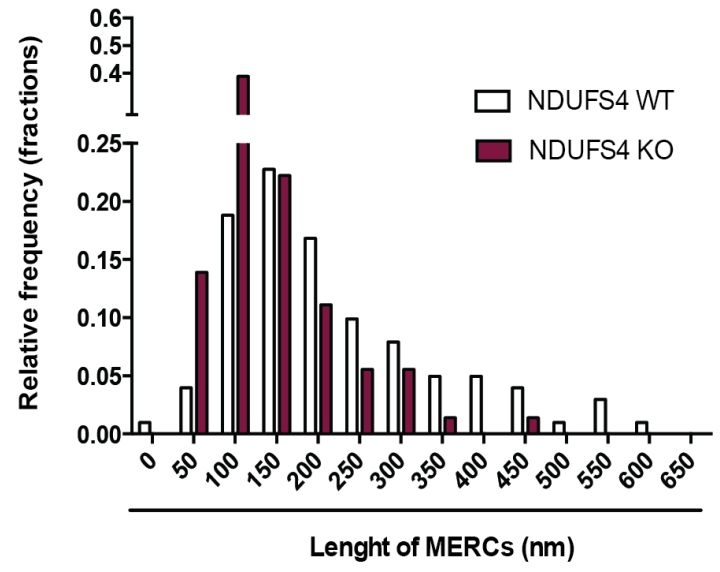

Figure 36 | Increased length of ER in contact with mitochondria in NDUFS4 KO cell model. (a) Morphometric analysis of ER length located at less than $30-\mathrm{nm}$ from mitochondria. Data represent mean \pm SEM of two independent experiments ( $n>300$ mitochondria per experiment). (b) Histogram reporting lengths quantified in (a). The differences were significant based on Mann-Whitney test, $* * * * P<0.0001$; SEM: standard error of the mean. 


\section{DISCUSSION}

Nowadays, mitochondrial signaling is not only associated with pathways triggered by mitochondria to affect gene expression but also, the response of other organelles to mitochondrial signaling, in the context of mitochondrial malfunction, has been recognized (Diogo et al., 2018; Fernandez-Mosquera et al., 2019). Reciprocally, the effect of other organelles on mitochondrial performance has been described (Peeters et al., 2015; Plotegher and Duchen, 2017; Raben et al., 2012; Selak et al., 2000; Yambire et al., 2019). Mitochondria interact with other organelles via signaling pathways or by physical interaction. The physical interaction between organelles are specialized areas in the cell where organelles directly contact each other, which are also known as contact sites (Scorrano et al., 2019). In this thesis, the main focus was to study the interaction between mitochondria and ER in the context of mitochondrial malfunction, and how these contact sites can be regulated by signaling pathways.

\subsection{Chronic and acute mitochondrial dysfunction differentially regulate} MERCS

Several contact sites have been described, and in the cell mitochondria make contact with different organelles, including lysosomes, peroxisomes, ER and lipid droplets (Klecker et al., 2014; Wong et al., 2018). The ER-mitochondria communication was the first discovered contact site, and the most studied organelle interaction, which has also been implicated in several cellular functions (Copeland, 1959). However, ER and mitochondria have distinct roles in the cell, and therefore for years, the impact of ER and mitochondrial dysfunction at cellular level was studied independently. Initially, the physiological role of MERCs was more associated with lipid transfer and calcium exchange between the two organelles (Kornmann et al., 2009; Rizzuto, 1998; Rizzuto et al., 2009). However, in the past several decades, with a growing interest in this field, the multifaceted role of MERCs in different cellular processes were discovered, and this role has been extended to new aspects of mitochondria and ER dynamics and function, such as active trigger of mitochondrial constriction and its subsequent division (Friedman et al., 2011). Given the molecular complexity of mitochondria-ER interface, it is plausible to hypothesize its implication in key signaling pathways, including the ones, which are activated after a mitochondrial stress and consequently have direct effects on MERCs formation and physiological function. 
In this study, as models of chronic mitochondrial malfunction, we used a stable knockdown of UQCRC1, a nuclear-encoded component of mitochondrial complex III, in HeLa cells, as well as MEFs from the NDUFS4-KO mouse with a deletion of one of the subunits of complex I of the ETC. These proteins are involved in the complex formation, and their deficiency leads to a failure in the proper formation of the complexes (Diaz et al., 2011; Gaspar et al., 2019). As expected, and also described previously by us, both models presented here have an impairment of mitochondrial performance, such as, loss of mitochondrial membrane potential and less oxygen consumption in basal and maximal respiration (Fernandez-Mosquera et al., 2019). Despite being an indirect measure of mitochondrial function, an increase of ROS production was observed, as also described in other cellular models associated with mitochondrial dysfunction (Lin and Beal, 2006; Wang et al., 2014). Notably, despite the different mutations in the respiratory chain complexes, an enrichment of MERCs was observed in both models of chronic mitochondrial malfunction. This finding underscore that changes in the number of MERCs due to chronic mitochondrial dysfunction are likely independent of the affected respiratory chain complex. Notwithstanding, other parameters analyzed, such as mitochondrial perimeter and length of MERCs, were not consistent in both models. This outcome suggests that complex-specific defects persist, given the localization of the defective proteins to different mitochondrial respiratory chain complexes, which can impact mitochondrial signaling and performance differently.

The ER-mitochondria communication is pivotal for the regulation of cellular homeostasis as well as, mitochondrial function. In fact, the key role of MERCs in the regulation of $\mathrm{Ca}^{2+}$ signaling, and consequently the impact on mitochondrial metabolism, bioenergetics and cell survival has been strongly established (Bravo-Sagua et al., 2017; Cárdenas et al., 2010; Rizzuto, 1998). Indeed, we show here an increase in basal mitochondrial $\mathrm{Ca}^{2+}$ in RC-kd cells. Therefore, the increase of MERCs can be a strategy of mitochondria to keep their functions by improving the $\mathrm{Ca}^{2+}$ transfer to mitochondria. For instance, the $\mathrm{Ca}^{2+}$ that is transferred to the mitochondria from ER allows the activation of some enzymes associated with TCA cycle, thus improving ATP production (Denton, 2009; Rossi et al., 2019). More recently, $\mathrm{Ca}^{2+}$ was also reported to modulate the ETC and $\mathrm{F}_{1} \mathrm{~F}_{0}$ ATP synthase, supporting an important role of $\mathrm{Ca}^{2+}$ on mitochondrial metabolism (Glancy et al., 2013; Rossi et al., 2019; Territo et al., 2017). However, it is important to mention that despite the enrichment of MERCs, an improvement in mitochondrial performance was not observed. This likely due to persistence of the mitochondrial dysfunction as the effects of a specific knock down in one of the complexes of the ETC may minimize the effect of MERCs 
increase, thereby making it less likely to see an improvement, similar to wild type levels, in mitochondrial function. Nevertheless, it is conceivable that MERCs enrichment is linked with a beneficial effect for mitochondria and consequently for the fate of the cell.

Furthermore, an increase of MERCs was also observed in some disease models in which mitochondrial function is also impaired as a consequence of the pathology (Arruda et al., 2014). Furthermore, alterations in MERCs formation in some neurodegenerative disorders, such as Alzheimer's disease has been shown (Area-Gomez and Schon, 2016; Hedskog et al., 2013; Schreiner et al., 2014).

In addition, to verify if the findings described pertained to only chronic mitochondrial malfunction, we employed a model of acute mitochondrial stress, by treating cells with antimycin A, a potent inhibitor of complex III of the ETC. EM analysis revealed that acute mitochondrial stress negatively affects the interaction between mitochondria and ER, observed by the reduction of the number of MERCs. Noteworthy, the decrease observed in the number of contact sites between mitochondria and ER was not a consequence of reduced mitochondrial perimeter since the average perimeter was increased in mitochondria involved in MERCs. One plausible explanation for this different outcome might be that the adaptive mechanism of increasing the number of MERCs and consequently improving mitochondrial performance is a long-term process. Hence, given the sudden and short duration of the mitochondrial stress in acute mitochondrial malfunction, it is likely that this adaptive mechanism is not triggered, and other pathways of improving mitochondrial function are engaged. Altogether, these findings underscore that the process of MERCs formation indeed responds to cellular signaling mechanisms and the regulation of MERCs responds differentially to mitochondrial stress depending on whether the stress is acute or chronic.

\subsection{AMPK signaling is regulated differentially in chronic and acute mitochondrial stress}

In the cell, the majority of ATP generation occurs in mitochondria, the sites of oxidative phosphorylation (Herzig and Shaw, 2018). Furthermore, it is important for the cells to manage their energy consumption depending on the nutrient availability, and on their capability to produce ATP. In some situations, ATP levels can decrease in the cell, making it essential for the cells to minimize energy consumption. In addition, under these conditions, processes aimed at restoring ATP levels for example, including activation of alternative pathways to generate 
energy, need to be employed (Herzig and Shaw, 2018). In higher eukaryotes, AMPK is a key player in a system, which allows the modulation of cellular metabolism depending on nutrient availability (Herzig and Shaw, 2018). AMPK activation promotes catabolism and decreases anabolism by phosphorylation of key proteins involved in multiple pathways, thereby restoring energy balance (Herzig and Shaw, 2018). Some aspects of mitochondrial biology and homeostasis are regulated by AMPK, such as stimulation of mitochondrial biogenesis, regulation of mitochondrial network, and regulation of mitochondrial quality control via regulation of autophagy and mitophagy (Kahn et al., 2005; Mihaylova and Shaw, 2011; Toyama et al., 2016). Moreover, it is known that AMPK is acutely induced under mitochondrial respiratory chain inhibition (Fernandez-Mosquera et al., 2019; Fernández-Mosquera et al., 2017; Herzig and Shaw, 2018).

Remarkably, in our model of chronic mitochondrial dysfunction (RC-kd), the AMPK pathway is repressed, which we previously observed in the context of another study (Fernandez-Mosquera et al., 2019). Besides, being a counterintuitive result, an explanation can be due to the increased protein levels of folliculin (FLCN) and folliculin interacting protein 1 (FNIP1) in the same model of chronic mitochondrial malfunction (Fernandez-Mosquera et al., 2019). Indeed, FLCN is a tumor-suppressor, and so far it is the only protein that is able to inhibit AMPK activity by forming heterotrimer complexes with FNIP1 and FNIP2 (Yan et al., 2014). On the other hand, and consistent with previous findings, our acute mitochondrial model displayed increased AMPK signaling. Indeed, using the same complex III inhibitor as in this study, Toyama and colleagues also reported AMPK activation in U2OS osteosarcoma cells, where extensive mitochondrial fragmentation was also detected (Toyama et al., 2016) further highlighting that AMPK activation following acute mitochondrial stress in independent of the cell type.

Interestingly, it can be appreciated from the findings that AMPK activity negatively correlates with the enrichment of MERCs. In chronic mitochondrial malfunction, the enrichment of MERCs is associated with repressed AMPK activity. Contrarily, acute mitochondrial stress is associated with increased AMPK activity and decreased number of MERCs. Taken together, this suggests that AMPK might be a potential key player in MERCs remodeling. 


\subsection{AMPK negatively affects MERCs formation}

As mentioned above, there is a relation between mitochondrial malfunction and the activation of AMPK signaling. Hence, to test if AMPK is indeed involved in the regulation of MERCs, we employed mouse embryonic fibroblasts lacking the two isoforms of the catalytic subunit of AMPK, $\alpha 1$ and $\alpha 2$ (AMPK $\alpha 1 \alpha 2$ double knock-out) (Laderoute et al., 2006). Several studies reported adaptive mechanisms in $\mathrm{AMPK \alpha}^{-/}$and $\mathrm{AMPK \alpha}^{-1-}$ mice by upregulation of the remaining AMPK $\alpha$ isoform, thus restoring and improving the response to metabolic stress (Jørgensen et al., 2004; Lieberthal et al., 2013). Consequently, it was crucial to develop a full AMPK $\alpha$ KO model in order to avoid this possible compensatory effect (O'Neill et al., 2011).

Our EM and fluorescence data suggested that the absence of AMPK activity is associated with increased amount of MERCs, demonstrating the negative regulation of AMPK on MERCs remodeling. This result was not due to changes in mitochondrial morphology or perimeter, since no morphological alterations were observed in cells lacking AMPK activity. The apparent effect of AMPK on mitochondria-ER communication can be seen as a strategy to keep both organelles far away from each other. Due to the robust catabolic response of cells to AMPK activation, it would be beneficial to move mitochondria elsewhere, since the proximity of mitochondria to ER, either due to the physical association or localization within the cell, can have negative effects on mitochondrial performance. In fact, it is known that in some conditions, such as in hepatocytes from obese mice, an increase of MERCs is associated with mitochondrial $\mathrm{Ca}^{2+}$ overload and higher mitochondrial ROS production. These effects were ameliorated by silencing PACS2 and $\mathrm{IP}_{3} \mathrm{R} 1$ (Arruda et al., 2014), suggesting that the higher proximity of mitochondria to ER could negatively impact mitochondrial performance. Additionally, these findings support the importance of tightly regulating MERCSs for the control of functional interactions between mitochondria and ER. Moreover, the increase of the number of MERCs was consisting with a slight increase on mitochondrial $\mathrm{Ca}^{2+}$ levels in AMPK-DKO cells, which could suggest that the effect observed is more structural than functional. In fact, it is known the ER plays a role by marking the possible constriction sites in mitochondria for fission (Friedman et al., 2011), thus contributing spuriously to the increase of functional MERCs until the fission process is completed. Additionally, a close relation between AMPK and the mitochondrial fission is known, since this kinase is able to activate some of the mitochondrial fission factors (Ducommun et al., 2015; Toyama et al., 2016). Altogether, cells lacking AMPK have repressed mitochondrial fission, suggesting the continued presence of ER close to mitochondria, which results in an increase of the number of MERCs. Taken together, our results support the conclusion that a bidirectional 
effect of AMPK exists, by regulating antagonistically the formation of MERCs depending on mitochondrial stress.

\subsection{The effect of AMPK on MERCs regulation is mediated by MFF}

In order to understand the mechanism of how AMPK regulates MERCs, we reasoned that an AMPK target must play a crucial role in MERCs. Here, we found out that MFF fits such a target since it was the only protein that scored after crossing published publicly available data sets of AMPK targets (Egan et al., 2011b; Gwinn et al., 2008) and MAM proteome (Hung et al., 2017). MFF localizes to mitochondria, where it functions as a receptor for DRP1, which catalyzes membrane scission during the process of mitochondrial fission (Losón et al., 2013; Otera et al., 2010; Shen et al., 2014; Toyama et al., 2016). In fact, in our subcellular fractionation results, we confirmed the presence of MFF in mitochondrial fractions. Interestingly in 'pure mitochondrial' fractions its amount was robustly decreased while increased levels were observed in the cytoplasm in AMPK-DKO cells. Altogether, this suggests that MFF phosphorylation by AMPK is required for its localization to mitochondria. Consistently, it was proved that the participation of MFF in mitochondrial fission is highly regulated by AMPK (Toyama et al., 2016). Notably, we found an enrichment of MERCs in cells lacking MFF-KO, providing evidence that MFF indeed is involved in repressing MERCs formation. Remarkably, the average mitochondrial perimeter of those involved in MERCs was not changed.

The elegant study from Toyama and colleagues demonstrated the role of AMPK on mitochondrial fission via its activity on MFF (Toyama et al., 2016). We took advantage of the remarkable manipulation of MFF phosphorylation in this study to elucidate how AMPK activity is coupled to MFF in remodeling MERCs, by expressing WT MFF, a non-phosphorylatable Ser155Ala-Ser172Ala (SA2) mutant MFF, as well as, a phospho-mimetic Ser155Asp-Ser172Asp mutant (SD2) in AMPK-DKO cells. Consistent with the previous study, the SD2 MFF mutant displayed gain-of-function activity when introduced into AMPK-DKO MEFs, displaying shortened mitochondria comparable with those expressing WT MFF (Toyama et al., 2016).

In the context of MERCs, our EM data shows that the phospho-mimetic isoform of MFF (SD2 MFF) normalizes the mitochondria-ER contact sites in cells lacking AMPK activity, suggesting that the effect of AMPK on MERCs is mediated by MFF. This result is consistent with the idea that the increased number of MERCs observed due to genetic absence of AMPK or absence of AMPK activity, has more a structural role rather than functional. Since, our data show that increasing 
MFF activity prevents the increase in contacts observed in AMPK-DKO MEFs, this effect might suggest that the re-activation of the mitochondrial fission process by more interaction of DRP1 with MFF completes the mitochondrial fission, thus minimizing the requirement for ER contacts to mark constriction sites on mitochondria.

Previously, the role of MERCs on mitochondrial fission was shown, in which ER tubules wrap around mitochondria to mark the fission sites (Friedman et al., 2011; Murley et al., 2013). Remarkably, MFF also localizes in these constriction regions to mark the sites where DRP1 will be recruited in order to trigger membrane scission (Friedman et al., 2011). From our data, we conclude that MFF seems to be involved in both mitochondrial fission and contact sites. In the absence of or diminished AMPK signaling, or when MFF is removed, mitochondrial fission is repressed, resulting in an increased proportion of long mitochondria. It seems the AMPK-MFF axis tightly regulates to linked processes: mitochondrial fission and from our data mitochondriaER contact sites. Notwithstanding, this study could not establish a direct role of AMPK as a structural tether between both organelles. However, it is also important to keep in mind that it is rather difficult to appreciate the role of a single tethering pair or molecule in remodeling MERCs. This is likely due to the large list of tethering proteins, which have been discovered to be associated with MERCs (Hung et al., 2017) and further justified by the findings that it is virtually impossible to eliminate a contact just by deleting any tether pair (Scorrano et al., 2019). Despite these limitations, here we demonstrated that signaling pathways are able to regulate MERCs, in particular AMPK via MFF.

Taken together, it seems that the association of mitochondria with the ER is another aspect of mitochondrial biology that is regulated by AMPK. The importance of AMPK in cell metabolism is very well characterized. For instance, AMPK is known to play a role in the upregulation of genes involved in mitochondrial biogenesis in the presence of mitochondrial dysfunction (Garcia-Roves et al., 2008; Herzig and Shaw, 2018; Jager et al., 2007) or to promote mitochondrial quality control via the removal of damaged mitochondrial through its regulation of the autophagy process (Egan et al., 2011a; Mihaylova and Shaw, 2011). However, in our model of chronic mitochondrial malfunction AMPK activity is repressed, and thus unable to activate adaptive mechanisms to improve mitochondrial quality. Therefore, it seems rational that the lack of AMPK will be couple to the regulation of a mechanism of increasing MERCs in the chronic mitochondrial model, as "backup" mechanism to increase mitochondria performance. Consistently, the acute mitochondrial stress model, which activates AMPK signaling, is likely able to engage pathways that trigger mitochondrial biogenesis and/or mitophagy, and would not 
require this "backup" mechanism, leading to its suppression. In a recent paper from our group, using the same model of chronic mitochondrial malfunction, defects in lysosomal function and consequently impaired autophagy as a consequence of mitochondrial dysfunction (FernandezMosquera et al., 2019) were observed. These findings suggest that maintenance of mitochondrial quality via mitophagy was impaired and highlight the crucial need for a mechanism to increase mitochondrial function to support survival. The increase of MERCs via the AMPK-MFF axis seems to be the missing 'wild card'. 


\section{Summary and conclusion}

Eukaryotes have a distribution of cellular functions in organelles, including the nucleus, mitochondrion, ER, or lysosomes. The existence of inter-organelle communication, as well as its role in several cellular functions, is becoming clear. In addition, organelle interplay is essential to maintain organelle homeostasis, and contributes to some pathologies that arise with organelle malfunction. Thus, understanding the crosstalk between organelles is a timely aspect of cell biology. During several years the impact of mitochondrial and ER dysfunction in the cell was studied independently due to the distinct primary roles of these organelles in the cell. Nevertheless, their interaction and capability to regulate the functions of each other has been recognized for decades. Furthermore, the effects of mitochondrial signaling, in the context of mitochondrial dysfunction, on other organelles has been described. Reciprocally, how defects in other organelles affect mitochondrial performance has also been characterized. However, evidence of how mitochondrial dysfunction can affect MERCs is little.

The outcomes of this study broaden our understanding of the mechanisms of communication between mitochondria and ER, in a context of mitochondrial dysfunction. This thesis addresses the effect of mitochondrial malfunction, in particular from respiratory chain stress, on MERCs formation and function. Here, it was shown that acute and chronic mitochondrial malfunction stresses have differential outcomes on MERCs formation. Interestingly, the AMPK signaling node seems to be the most important pathway mediating the MERCs formation in the context of mitochondrial malfunction. Moreover, AMPK responses differentially for the different mitochondrial malfunction stresses. In fact, the AMPK regulation seems to be dependent by MFF activity, since we observed that modulation of MFF activity in cells lacking AMPK restores the number of MERCs. Hence, we show here that AMPK is a negative regulatory kinase of the MERCS formation via the action of MFF (Figure 37).

To conclude, our findings reveal that MERCs respond to mitochondrial respiratory chain malfunction in a defined manner through a well-understood signaling pathway, which may contribute to a better understanding of pathological conditions linked to MERCS. 


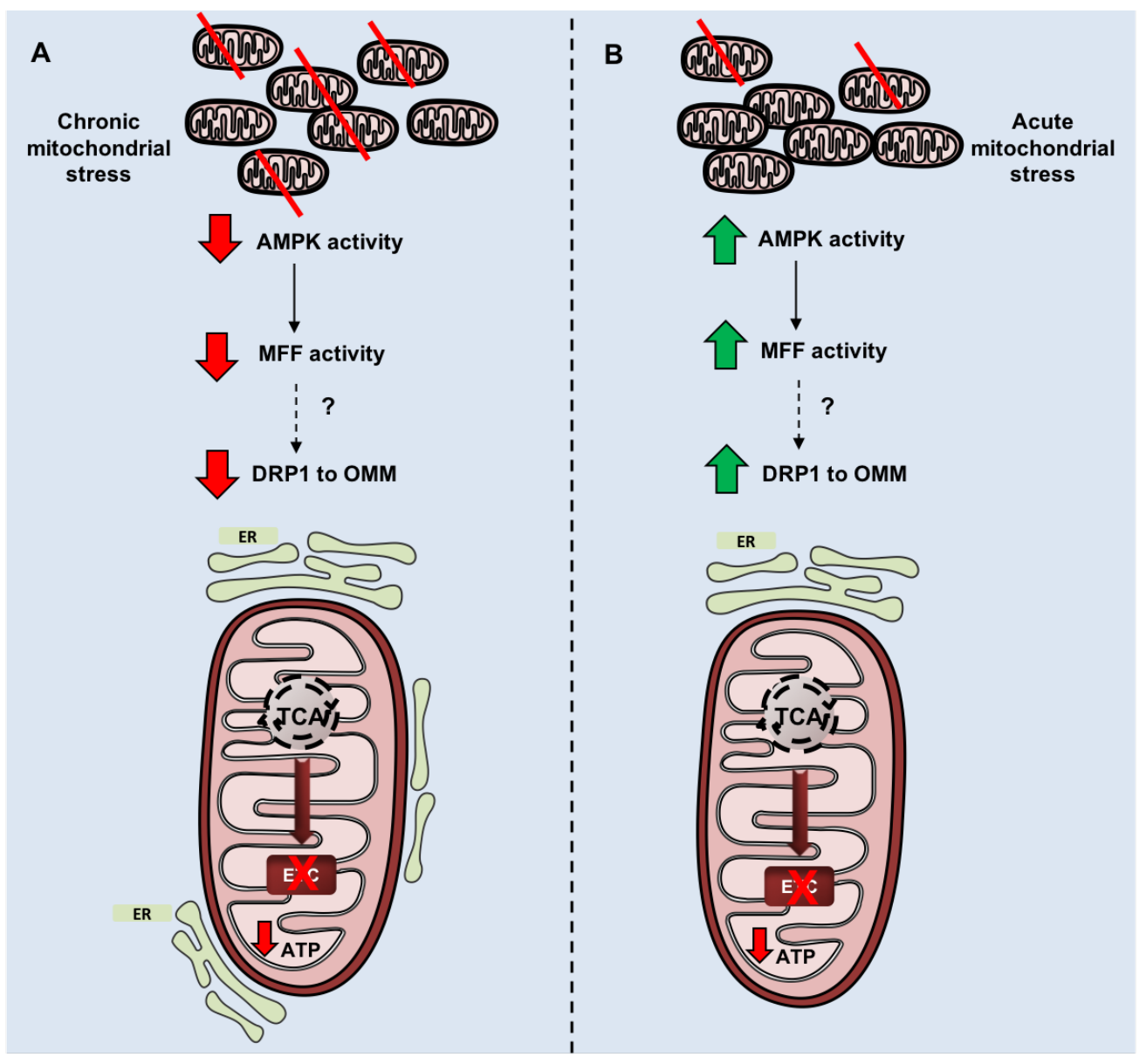

Figure 37 - Proposed model of mitochondria-ER communication in chronic and acute mitochondrial stress. AMPK responds differentially to the different mitochondrial malfunction stresses, (A) chronic mitochondrial malfunction and (B) acute mitochondrial dysfunction. AMPK regulation of MERCs seems to be dependent on MFF activity. Consequently, chronic and acute mitochondrial malfunction stresses have differential outcomes on MERCs number. 


\section{REFERENCES}

Adler, E., Euler, H. V., Günther, G., and Plass, M. (1939). iso Citric dehydrogenase and glutamic acid synthesis in animal tissues. Biochem. J. 33, 1028-1045.

Ahmadian, M., Abbott, M.J., Tang, T., Hudak, C.S.S., Kim, Y., Bruss, M., Hellerstein, M.K., Lee, H.Y., Samuel, V.T., Shulman, G.I., et al. (2011). Desnutrin/ATGL is regulated by AMPK and is required for a brown adipose phenotype. Cell Metab. 13, 739-748.

Anderson, D.J., and Hetzer, M.W. (2007). Nuclear envelope formation by chromatin-mediated reorganization of the endoplasmic reticulum. Nat. Cell Biol. 9, 1160-1166.

Area-Gomez, E. (2014). Assessing the function of mitochondria-associated ER membranes. In Methods in Enzymology, p.

Area-Gomez, E., and Schon, E.A. (2016). Mitochondria-associated ER membranes and Alzheimer disease. Curr. Opin. Genet. Dev. 38, 90-96.

Arruda, A.P., Pers, B.M., Parlakgül, G., Güney, E., Inouye, K., and Hotamisligil, G.S. (2014).

Chronic enrichment of hepatic endoplasmic reticulum-mitochondria contact leads to mitochondrial dysfunction in obesity. Nat. Med. 20, 1427-1435.

Ban, T., Ishihara, T., Kohno, H., Saita, S., Ichimura, A., Maenaka, K., Oka, T., Mihara, K., and Ishihara, N. (2017). Molecular basis of selective mitochondrial fusion by heterotypic action between OPA1 and cardiolipin. Nat. Cell Biol. 19, 856-863.

Barlowe, C. (2009). Atlasin GTPases Shape Up ER Networks. Dev. Cell 17, 157-158.

Baughman, J.M., Perocchi, F., Girgis, H.S., Plovanich, M., Belcher-Timme, C.A., Sancak, Y., Bao, X.R., Strittmatter, L., Goldberger, O., Bogorad, R.L., et al. (2011). Integrative genomics identifies MCU as an essential component of the mitochondrial calcium uniporter. Nature 476, 341-345.

Bernard-Marissal, N., Médard, J.-J., Azzedine, H., and Chrast, R. (2015). Dysfunction in endoplasmic reticulum-mitochondria crosstalk underlies SIGMAR1 loss of function mediated motor neuron degeneration. Brain 138, 875-890.

Berridge, M.J. (2002). The endoplasmic reticulum: a multifunctional signaling organelle. Cell Calcium 32, 235-249.

Booth, D.M., Enyedi, B., Geiszt, M., Várnai, P., and Hajnóczky, G. (2016). Redox Nanodomains Are Induced by and Control Calcium Signaling at the ER-Mitochondrial Interface. Mol. Cell 63, $240-248$. 
Bravo-Sagua, R., E. Rodriguez, A., Kuzmicic, J., Gutierrez, T., Lopez-Crisosto, C., Quiroga, C., Diaz-Elizondo, J., Chiong, M., G. Gillette, T., A. Rothermel, B., et al. (2013). Cell Death and Survival Through the Endoplasmic Reticulum- Mitochondrial Axis. Curr. Mol. Med. 13, 317329.

Bravo-Sagua, R., Parra, V., López-Crisosto, C., Díaz, P., Quest, A.F.G., and Lavandero, S. (2017). Calcium Transport and Signaling in Mitochondria. In Comprehensive Physiology, (Hoboken, NJ, USA: John Wiley \& Sons, Inc.), pp. 623-634.

Bravo, R., Vicencio, J.M., Parra, V., Troncoso, R., Munoz, J.P., Bui, M., Quiroga, C., Rodriguez, A.E., Verdejo, H.E., Ferreira, J., et al. (2011). Increased ER-mitochondrial coupling promotes mitochondrial respiration and bioenergetics during early phases of ER stress. J. Cell Sci. 124, 2511-2511.

Bravo, R., Parra, V., Gatica, D., Rodriguez, A.E., Torrealba, N., Paredes, F., Wang, Z. V., Zorzano, A., Hill, J.A., Jaimovich, E., et al. (2013). Endoplasmic Reticulum and the Unfolded Protein Response. In International Review of Cell and Molecular Biology, pp. 215-290.

De Brito, O.M., and Scorrano, L. (2008). Mitofusin 2 tethers endoplasmic reticulum to mitochondria. Nature 456, 605-610.

Bui, M., Gilady, S.Y., Fitzsimmons, R.E.B., Benson, M.D., Lynes, E.M., Gesson, K., Alto, N.M., Strack, S., Scott, J.D., and Simmen, T. (2010). Rab32 Modulates Apoptosis Onset and Mitochondria-associated Membrane (MAM) Properties. J. Biol. Chem. 285, 31590-31602.

Calì, T., Ottolini, D., Negro, A., and Brini, M. (2012). $\alpha$-Synuclein Controls Mitochondrial Calcium Homeostasis by Enhancing Endoplasmic Reticulum-Mitochondria Interactions. J. Biol. Chem. 287, 17914-17929.

Calì, T., Ottolini, D., Negro, A., and Brini, M. (2013). Enhanced parkin levels favor ERmitochondria crosstalk and guarantee $\mathrm{Ca} 2+$ transfer to sustain cell bioenergetics. Biochim. Biophys. Acta - Mol. Basis Dis. 1832, 495-508.

Cao, Y.-L., Meng, S., Chen, Y., Feng, J.-X., Gu, D.-D., Yu, B., Li, Y.-J., Yang, J.-Y., Liao, S., Chan, D.C., et al. (2017). MFN1 structures reveal nucleotide-triggered dimerization critical for mitochondrial fusion. Nature 542, 372-376.

Cárdenas, C., Miller, R.A., Smith, I., Bui, T., Molgó, J., Müller, M., Vais, H., Cheung, K.-H., Yang, J., Parker, I., et al. (2010). Essential Regulation of Cell Bioenergetics by Constitutive InsP3 Receptor Ca2+ Transfer to Mitochondria. Cell 142, 270-283. 
Cereghetti, G.M., Stangherlin, a, Martins de Brito, O., Chang, C.R., Blackstone, C., Bernardi, P., and Scorrano, L. (2008). Dephosphorylation by calcineurin regulates translocation of Drp1 to mitochondria. Proc. Natl. Acad. Sci. U. S. A. 105, 15803-15808.

Chakrabarti, R., Ji, W.-K., Stan, R. V., de Juan Sanz, J., Ryan, T.A., and Higgs, H.N. (2018). INF2mediated actin polymerization at the ER stimulates mitochondrial calcium uptake, inner membrane constriction, and division. J. Cell Biol. 217, 251-268.

Chan, D.C. (2012). Fusion and Fission: Interlinked Processes Critical for Mitochondrial Health. Annu. Rev. Genet. 46, 265-287.

Chandel, N.S. (2014). Mitochondria as signaling organelles. BMC Biol. 12, 34.

Chandel, N.S. (2015). Evolution of Mitochondria as Signaling Organelles. Cell Metab. 22, 204206.

Chandel, N.S., Maltepe, E., Goldwasser, E., Mathieu, C.E., Simon, M.C., and Schumacker, P.T. (1998). Mitochondrial reactive oxygen species trigger hypoxia-induced transcription. Proc. Natl. Acad. Sci. 95, 11715-11720.

Chandel, N.S., McClintock, D.S., Feliciano, C.E., Wood, T.M., Melendez, J.A., Rodriguez, A.M., and Schumacker, P.T. (2000). Reactive Oxygen Species Generated at Mitochondrial Complex III Stabilize Hypoxia-inducible Factor-1 $\alpha$ during Hypoxia. J. Biol. Chem. 275, 25130-25138.

Chazotte, B. (2011). Labeling mitochondria with JC-1. Cold Spring Harb. Protoc.

Chen, H., Detmer, S.A., Ewald, A.J., Griffin, E.E., Fraser, S.E., and Chan, D.C. (2003). Mitofusins Mfn1 and Mfn2 coordinately regulate mitochondrial fusion and are essential for embryonic development. J. Cell Biol. 160, 189-200.

Cho, I.-T., Adelmant, G., Lim, Y., Marto, J.A., Cho, G., and Golden, J.A. (2017). Ascorbate peroxidase proximity labeling coupled with biochemical fractionation identifies promoters of endoplasmic reticulum-mitochondrial contacts. J. Biol. Chem. 292, 16382-16392.

Chu, C.T. (2010). A pivotal role for PINK1 and autophagy in mitochondrial quality control: Implications for Parkinson disease. Hum. Mol. Genet.

Cieri, D., Vicario, M., Vallese, F., D’Orsi, B., Berto, P., Grinzato, A., Catoni, C., De Stefani, D., Rizzuto, R., Brini, M., et al. (2018). Tau localises within mitochondrial sub-compartments and its caspase cleavage affects ER-mitochondria interactions and cellular $\mathrm{Ca} 2+$ handling. Biochim. Biophys. Acta - Mol. Basis Dis. 1864, 3247-3256.

Clapham, D.E. (2005). Calcium Signaling (CRC Press). 
Clayton, D.A., and Shadel, G.S. (2015). Isolation of Mitochondria from Cells and Tissues. 147151.

Clayton, D.A., Shadel, G.S., Clayton, D.A., and Shadel, G.S. (2014). Purification of Mitochondria by Sucrose Step Density Gradient Centrifugation Purification of Mitochondria by Sucrose Step Density Gradient Centrifugation.

Cohen, S., Valm, A.M., and Lippincott-Schwartz, J. (2018). Interacting organelles. Curr. Opin. Cell Biol. 53, 84-91.

Colombini, M. (1980). Pore size and properties of channels from mitochondria isolated fromNeurospora crassa. J. Membr. Biol. 53, 79-84.

Copeland, D.E. (1959). An Association between Mitochondria and the Endoplasmic Reticulum in Cells of the Pseudobranch Gland of a Teleost. J. Cell Biol. 5, 393-396.

Costello, J.L., Castro, I.G., Hacker, C., Schrader, T.A., Metz, J., Zeuschner, D., Azadi, A.S., Godinho, L.F., Costina, V., Findeisen, P., et al. (2017). ACBD5 and VAPB mediate membrane associations between peroxisomes and the ER. J. Cell Biol. 216, 331-342.

CROMPTON, M. (1999). The mitochondrial permeability transition pore and its role in cell death. Biochem. J. 341, 233-249.

Crozet, P., Margalha, L., Confraria, A., Rodrigues, A., Martinho, C., Adamo, M., Elias, C.A., and Baena-GonzÃilez, E. (2014). Mechanisms of regulation of SNF1/AMPK/SnRK1 protein kinases. Front. Plant Sci. 5.

Csordás, G., Renken, C., Várnai, P., Walter, L., Weaver, D., Buttle, K.F., Balla, T., Mannella, C.A., and Hajnóczky, G. (2006). Structural and functional features and significance of the physical linkage between ER and mitochondria. J. Cell Biol. 174, 915-921.

Csordás, G., Várnai, P., Golenár, T., Roy, S., Purkins, G., Schneider, T.G., Balla, T., and Hajnóczky, G. (2010). Imaging Interorganelle Contacts and Local Calcium Dynamics at the ERMitochondrial Interface. Mol. Cell 39, 121-132.

Csordás, G., Weaver, D., and Hajnóczky, G. (2018). Endoplasmic Reticulum-Mitochondrial Contactology: Structure and Signaling Functions. Trends Cell Biol. 28, 523-540.

Cui, Z., Jean, E., Chenii, M.H., Voelker, D.R., and Q, D.E.V. (1993). Cloning and Expression of a Novel Phosphatidylethanolamine N-Methyltransferase. J. Biol. Chem. 268, 16655-16663.

Davies, V.J., Hollins, A.J., Piechota, M.J., Yip, W., Davies, J.R., White, K.E., Nicols, P.P., Boulton, M.E., and Votruba, M. (2007). Opa1 deficiency in a mouse model of autosomal dominant optic 
atrophy impairs mitochondrial morphology, optic nerve structure and visual function. Hum. Mol. Genet. 16, 1307-1318.

Deas, E., Wood, N.W., and Plun-Favreau, H. (2011). Mitophagy and Parkinson's disease: The PINK1-parkin link. Biochim. Biophys. Acta - Mol. Cell Res. 1813, 623-633.

Denton, R.M. (2009). Regulation of mitochondrial dehydrogenases by calcium ions. Biochim. Biophys. Acta - Bioenerg. 1787, 1309-1316.

Diaz, F., Kotarsky, H., Fellman, V., and Moraes, C.T. (2011). Mitochondrial disorders caused by mutations in respiratory chain assembly factors. Semin. Fetal Neonatal Med. 16, 197-204.

DiMauro, S., and Schon, E.A. (2008). Mitochondrial Disorders in the Nervous System. Annu. Rev. Neurosci. 31, 91-123.

Diogo, C. V., Yambire, K.F., Fernández Mosquera, L., Branco F., T., and Raimundo, N. (2018). Mitochondrial adventures at the organelle society. Biochem. Biophys. Res. Commun. 500, 8793.

Doghman-Bouguerra, M., and Lalli, E. (2019). ER-mitochondria interactions: Both strength and weakness within cancer cells. Biochim. Biophys. Acta - Mol. Cell Res. 1866, 650-662.

Ducommun, S., Deak, M., Sumpton, D., Ford, R.J., Núñez Galindo, A., Kussmann, M., Viollet, B., Steinberg, G.R., Foretz, M., Dayon, L., et al. (2015). Motif affinity and mass spectrometry proteomic approach for the discovery of cellular AMPK targets: Identification of mitochondrial fission factor as a new AMPK substrate. Cell. Signal. 27, 978-988.

Egan, D.F., Shackelford, D.B., Mihaylova, M.M., Gelino, S., Kohnz, R.A., Mair, W., Vasquez, D.S., Joshi, A., Gwinn, D.M., Taylor, R., et al. (2011a). Phosphorylation of ULK1 (hATG1) by AMPactivated protein kinase connects energy sensing to mitophagy. Science (80-. ).

Egan, D.F., Shackelford, D.B., Mihaylova, M.M., Gelino, S., Kohnz, R.A., Mair, W., Vasquez, D.S., Joshi, A., Gwinn, D.M., Taylor, R., et al. (2011b). Phosphorylation of ULK1 (hATG1) by AMPActivated Protein Kinase Connects Energy Sensing to Mitophagy. Science (80-. ). 331, 456-461. Eisen, A. (1985). Source and sinks for the calcium released during fertilization of single sea urchin eggs. J. Cell Biol. 100, 1522-1527.

Eisenberg-Bord, M., Shai, N., Schuldiner, M., and Bohnert, M. (2016). A Tether Is a Tether Is a Tether: Tethering at Membrane Contact Sites. Dev. Cell 39, 395-409.

Elbaz-Alon, Y., Eisenberg-Bord, M., Shinder, V., Stiller, S.B., Shimoni, E., Wiedemann, N., Geiger, T., and Schuldiner, M. (2015). Lam6 Regulates the Extent of Contacts between 
Organelles. Cell Rep. 12, 7-14.

Elefantova, K., Lakatos, B., Kubickova, J., Sulova, Z., and Breier, A. (2018). Detection of the Mitochondrial Membrane Potential by the Cationic Dye JC-1 in L1210 Cells with Massive Overexpression of the Plasma Membrane ABCB1 Drug Transporter. Int. J. Mol. Sci. 19, 1985.

English, A.R., and Voeltz, G.K. (2013). Endoplasmic Reticulum Structure and Interconnections with Other Organelles. Cold Spring Harb. Perspect. Biol. 5, a013227-a013227.

Eura, Y. (2003). Two Mitofusin Proteins, Mammalian Homologues of FZO, with Distinct Functions Are Both Required for Mitochondrial Fusion. J. Biochem. 134, 333-344.

Fagone, P., and Jackowski, S. (2009). Membrane phospholipid synthesis and endoplasmic reticulum function. J. Lipid Res. 50, S311-S316.

Farhan, H., and Hauri, H.-P. (2009). Membrane Biogenesis: Networking at the ER with Atlastin. Curr. Biol. 19, R906-R908.

Fernandez-Mosquera, L., Yambire, K.F., Couto, R., Pereyra, L., Pabis, K., Ponsford, A.H., Diogo, C. V., Stagi, M., Milosevic, I., and Raimundo, N. (2019). Mitochondrial respiratory chain deficiency inhibits lysosomal hydrolysis. Autophagy 15, 1572-1591.

Fernández-Mosquera, L., Diogo, C. V., Yambire, K.F., Santos, G.L., Luna Sánchez, M., Bénit, P., Rustin, P., Lopez, L.C., Milosevic, I., and Raimundo, N. (2017). Acute and chronic mitochondrial respiratory chain deficiency differentially regulate lysosomal biogenesis. Sci. Rep. 7, 45076.

Filadi, R., Greotti, E., Turacchio, G., Luini, A., Pozzan, T., and Pizzo, P. (2015). Mitofusin 2 ablation increases endoplasmic reticulum-mitochondria coupling. Proc. Natl. Acad. Sci. 112, E2174-E2181.

Filadi, R., Greotti, E., and Pizzo, P. (2018). Highlighting the endoplasmic reticulummitochondria connection: Focus on Mitofusin 2. Pharmacol. Res. 128, 42-51.

Fill, M., and Copello, J.A. (2002). Ryanodine Receptor Calcium Release Channels. Physiol. Rev. $82,893-922$.

Fonseca, T.B., Sánchez-Guerrero, Á., Milosevic, I., and Raimundo, N. (2019). Mitochondrial fission requires DRP1 but not dynamins. Nature 570, E34-E42.

Foskett, J.K., White, C., Cheung, K.-H., and Mak, D.-O.D. (2007). Inositol Trisphosphate Receptor Ca 2+ Release Channels . Physiol. Rev. 87, 593-658.

Friedman, J.R., and Voeltz, G.K. (2011). The ER in 3D: A multifunctional dynamic membrane 
network. Trends Cell Biol. 21, 709-717.

Friedman, J.R., Webster, B.M., Mastronarde, D.N., Verhey, K.J., and Voeltz, G.K. (2010). ER sliding dynamics and ER-mitochondrial contacts occur on acetylated microtubules. J. Cell Biol. 190, 363-375.

Friedman, J.R., Lackner, L.L., West, M., DiBenedetto, J.R., Nunnari, J., and Voeltz, G.K. (2011). ER tubules mark sites of mitochondrial division. Science (80-. ). 334, 358-362.

Gallagher, S.R., and li, S. (2006). One-dimensional SDS gel electrophoresis of proteins. Curr. Protoc. Mol. Biol.

Galmes, R., Houcine, A., van Vliet, A.R., Agostinis, P., Jackson, C.L., and Giordano, F. (2016). ORP5/ORP8 localize to endoplasmic reticulum-mitochondria contacts and are involved in mitochondrial function. EMBO Rep. 17, 800-810.

Gandre-Babbe, S., and van der Bliek, A.M. (2008). The novel tail-anchored membrane protein Mff controls mitochondrial and peroxisomal fission in mammalian cells. Mol. Biol. Cell 19, $2402-2412$.

Garcia-Roves, P.M., Osler, M.E., Holmström, M.H., and Zierath, J.R. (2008). Gain-of-function R225Q mutation in AMP-activated protein kinase $\gamma 3$ subunit increases mitochondrial biogenesis in glycolytic skeletal muscle. J. Biol. Chem. 283, 35724-35734.

Gaspar, B.L., Vasishta, R.K., and Radotra, B.D. (2019). Mitochondrial Myopathies and Related Diseases. In Myopathology, (Singapore: Springer Singapore), pp. 165-200.

Gautier, C.A., Erpapazoglou, Z., Mouton-Liger, F., Muriel, M.P., Cormier, F., Bigou, S., Duffaure, S., Girard, M., Foret, B., lannielli, A., et al. (2016). The endoplasmic reticulum-mitochondria interface is perturbed in PARK2 knockout mice and patients with PARK2 mutations. Hum. Mol. Genet. ddw148.

Gelmetti, V., De Rosa, P., Torosantucci, L., Marini, E.S., Romagnoli, A., Di Rienzo, M., Arena, G., Vignone, D., Fimia, G.M., and Valente, E.M. (2017). PINK1 and BECN1 relocalize at mitochondria-associated membranes during mitophagy and promote ER-mitochondria tethering and autophagosome formation. Autophagy 13, 654-669.

Giacomello, M., Drago, I., Bortolozzi, M., Scorzeto, M., Gianelle, A., Pizzo, P., and Pozzan, T. (2010). Ca2+ Hot Spots on the Mitochondrial Surface Are Generated by Ca2+ Mobilization from Stores, but Not by Activation of Store-Operated Ca2+ Channels. Mol. Cell 38, 280-290.

Gibhardt, C.S., Zimmermann, K.M., Zhang, X., Belousov, V. V, and Bogeski, I. (2016). Cell 
Calcium Imaging calcium and redox signals using genetically encoded fluorescent indicators. Cell Calcium 60, 55-64.

Gilmore, R. (1982). Protein translocation across the endoplasmic reticulum. I. Detection in the microsomal membrane of a receptor for the signal recognition particle. J. Cell Biol. 95, 463469.

Giordano, F. (2018). Non-vesicular lipid trafficking at the endoplasmic reticulum mitochondria interface. 1-16.

Glancy, B., Willis, W.T., Chess, D.J., and Balaban, R.S. (2013). Effect of calcium on the oxidative phosphorylation cascade in skeletal muscle mitochondria. Biochemistry 52, 2793-2809.

Gomes, L.C., Benedetto, G. Di, and Scorrano, L. (2011a). During autophagy mitochondria elongate, are spared from degradation and sustain cell viability. Nat. Cell Biol. 13, 589-598.

Gomes, L.C., Di Benedetto, G., and Scorrano, L. (2011b). Essential amino acids and glutamine regulate induction of mitochondrial elongation during autophagy. Cell Cycle 10, 2635-2639.

Gomez-Suaga, P., Paillusson, S., Stoica, R., Noble, W., Hanger, D.P., and Miller, C.C.J. (2017). The ER-Mitochondria Tethering Complex VAPB-PTPIP51 Regulates Autophagy. Curr. Biol. 27, 371-385.

Gómez-Suaga, P., Bravo-San Pedro, J.M., González-Polo, R.A., Fuentes, J.M., and Niso-Santano, M. (2018). ER-mitochondria signaling in Parkinson's disease. Cell Death Dis. 9, 337.

Grigoriev, I., Gouveia, S.M., van der Vaart, B., Demmers, J., Smyth, J.T., Honnappa, S., Splinter, D., Steinmetz, M.O., Putney, J.W., Hoogenraad, C.C., et al. (2008). STIM1 Is a MT-Plus-EndTracking Protein Involved in Remodeling of the ER. Curr. Biol. 18, 177-182.

Guardia-Laguarta, C., Area-Gomez, E., Rub, C., Liu, Y., Magrane, J., Becker, D., Voos, W., Schon, E.A., and Przedborski, S. (2014). -Synuclein Is Localized to Mitochondria-Associated ER Membranes. J. Neurosci. 34, 249-259.

Gutiérrez, T., Parra, V., Troncoso, R., Pennanen, C., Contreras-Ferrat, A., Vasquez-Trincado, C., Morales, P.E., Lopez-Crisosto, C., Sotomayor-Flores, C., Chiong, M., et al. (2014). Alteration in mitochondrial $\mathrm{Ca} 2+$ uptake disrupts insulin signaling in hypertrophic cardiomyocytes. Cell Commun. Signal. 12, 68.

Gwinn, D.M., Shackelford, D.B., Egan, D.F., Mihaylova, M.M., Mery, A., Vasquez, D.S., Turk, B.E., and Shaw, R.J. (2008). AMPK Phosphorylation of Raptor Mediates a Metabolic Checkpoint. Mol. Cell 30, 214-226. 
Hamasaki, M., Furuta, N., Matsuda, A., Nezu, A., Yamamoto, A., Fujita, N., Oomori, H., Noda, T., Haraguchi, T., Hiraoka, Y., et al. (2013). Autophagosomes form at ER-mitochondria cHamasaki, M., Furuta, N., Matsuda, A., Nezu, A., Yamamoto, A., Fujita, N., Oomori, H., Noda, T., Haraguchi, T., Hiraoka, Y., et al. (2013). Autophagosomes form at ER-mitochondria contact sites. Nature 495, 389-393.o. Nature 495, 389-393.

Han, D., Antunes, F., Canali, R., Rettori, D., and Cadenas, E. (2003). Voltage-dependent anion channels control the release of the superoxide anion from mitochondria to cytosol. J. Biol. Chem. 278, 5557-5563.

Hanada, K., Kumagai, K., Tomishige, N., and Yamaji, T. (2009). CERT-mediated trafficking of ceramide. Biochim. Biophys. Acta - Mol. Cell Biol. Lipids 1791, 684-691.

Hartl, F.U., and Hayer-Hartl, M. (2009). Converging concepts of protein folding in vitro and in vivo. Nat. Struct. Mol. Biol. 16, 574-581.

Hedskog, L., Pinho, C.M., Filadi, R., Ronnback, A., Hertwig, L., Wiehager, B., Larssen, P., Gellhaar, S., Sandebring, A., Westerlund, M., et al. (2013). Modulation of the endoplasmic reticulum-mitochondria interface in Alzheimer's disease and related models. Proc. Natl. Acad. Sci. 110, 7916-7921.

Helle, S.C.J., Kanfer, G., Kolar, K., Lang, A., Michel, A.H., and Kornmann, B. (2013). Organization and function of membrane contact sites. Biochim. Biophys. Acta - Mol. Cell Res. 1833, 25262541.

Herzig, S., and Shaw, R.J. (2018). AMPK: Guardian of metabolism and mitochondrial homeostasis. Nat. Rev. Mol. Cell Biol. 19.

Hirabayashi, Y., Kwon, S.-K., Paek, H., Pernice, W.M., Paul, M.A., Lee, J., Erfani, P., Raczkowski, A., Petrey, D.S., Pon, L.A., et al. (2017). ER-mitochondria tethering by PDZD8 regulates Ca2+ dynamics in mammalian neurons. Science (80-. ). 358, 623-630.

Hirota, Y., and Tanaka, Y. (2009). A small GTPase, human Rab32, is required for the formation of autophagic vacuoles under basal conditions. Cell. Mol. Life Sci. 66, 2913-2932.

Hoppins, S., Edlich, F., Cleland, M.M., Banerjee, S., McCaffery, J.M., Youle, R.J., and Nunnari, J. (2011). The Soluble Form of Bax Regulates Mitochondrial Fusion via MFN2 Homotypic Complexes. Mol. Cell 41, 150-160.

Hu, J., Shibata, Y., Zhu, P.-P., Voss, C., Rismanchi, N., Prinz, W.A., Rapoport, T.A., and Blackstone, C. (2009). A Class of Dynamin-like GTPases Involved in the Generation of the 
Tubular ER Network. Cell 138, 549-561.

Hua, R., Cheng, D., Coyaud, É., Freeman, S., Di Pietro, E., Wang, Y., Vissa, A., Yip, C.M., Fairn, G.D., Braverman, N., et al. (2017). VAPs and ACBD5 tether peroxisomes to the ER for peroxisome maintenance and lipid homeostasis. J. Cell Biol. 216, 367-377.

Hung, V., Lam, S.S., Udeshi, N.D., Svinkina, T., Guzman, G., Mootha, V.K., Carr, S.A., and Ting, A.Y. (2017). Proteomic mapping of cytosol-facing outer mitochondrial and ER membranes in living human cells by proximity biotinylation. Elife 6, 1-38.

Ingerman, E., Perkins, E.M., Marino, M., Mears, J.A., McCaffery, J.M., Hinshaw, J.E., and Nunnari, J. (2005). Dnm1 forms spirals that are structurally tailored to fit mitochondria. J. Cell Biol. 170, 1021-1027.

Ishihara, N., Nomura, M., Jofuku, A., Kato, H., Suzuki, S.O., Masuda, K., Otera, H., Nakanishi, Y., Nonaka, I., Goto, Y., et al. (2009). Mitochondrial fission factor Drp1 is essential for embryonic development and synapse formation in mice. Nat. Cell Biol. 11, 958-966.

Iwasawa, R., Mahul-Mellier, A.-L., Datler, C., Pazarentzos, E., and Grimm, S. (2011). Fis1 and Bap31 bridge the mitochondria-ER interface to establish a platform for apoptosis induction. EMBO J. 30, 556-568.

Jaffe, L.F. (1983). Sources of calcium in egg activation: A review and hypothesis. Dev. Biol. 99, 265-276.

Jager, S., Handschin, C., St.-Pierre, J., and Spiegelman, B.M. (2007). AMP-activated protein kinase (AMPK) action in skeletal muscle via direct phosphorylation of PGC-1. Proc. Natl. Acad. Sci. 104, 12017-12022.

Jørgensen, S.B., Viollet, B., Andreelli, F., Frøsig, C., Birk, J.B., Schjerling, P., Vaulont, S., Richter, E.A., and Wojtaszewski, J.F.P. (2004). Knockout of the $\alpha 2$ but Not $\alpha 1,5^{\prime}$-AMP-activated Protein Kinase Isoform Abolishes 5-Aminoimidazole-4-carboxamide-1- $\beta$-4-ribofuranoside- but Not Contraction-induced Glucose Uptake in Skeletal Muscle. J. Biol. Chem. 279, 1070-1079.

Kahn, B.B., Alquier, T., Carling, D., and Hardie, D.G. (2005). AMP-activated protein kinase: Ancient energy gauge provides clues to modern understanding of metabolism. Cell Metab. 1, $15-25$.

Kainu, V., Hermansson, M., Hänninen, S., Hokynar, K., and Somerharju, P. (2013). Import of phosphatidylserine to and export of phosphatidylethanolamine molecular species from mitochondria. Biochim. Biophys. Acta - Mol. Cell Biol. Lipids 1831, 429-437. 
Kamerkar, S.C., Kraus, F., Sharpe, A.J., Pucadyil, T.J., and Ryan, M.T. (2018). Dynamin-related protein 1 has membrane constricting and severing abilities sufficient for mitochondrial and peroxisomal fission. Nat. Commun. 9, 5239.

Kessler, R.J., Tyson, C.A., and Green, D.E. (1976). Mechanism of uncoupling in mitochondria: uncouplers as ionophores for cycling cations and protons. Proc. Natl. Acad. Sci. 73, 3141-3145. Kim, J., Kundu, M., Viollet, B., and Guan, K.-L. (2011). AMPK and mTOR regulate autophagy through direct phosphorylation of Ulk1. Nat. Cell Biol. 13, 132-141.

Kitada, T., Asakawa, S., Hattori, N., Matsumine, H., Yamamura, Y., Minoshima, S., Yokochi, M., Mizuno, Y., and Shimizu, N. (1998). Mutations in the parkin gene cause autosomal recessive juvenile parkinsonism. Nature 392, 605-608.

Klecker, T., Böckler, S., and Westermann, B. (2014). Making connections: Interorganelle contacts orchestrate mitochondrial behavior. Trends Cell Biol. 24, 537-545.

Klopfenstein, D.R.C., Kappeler, F., and Hauri, H.-P. (1998). A novel direct interaction of endoplasmic reticulum with microtubules. EMBO J. 17, 6168-6177.

Ko, A.-R., Hyun, H.-W., Min, S.-J., and Kim, J.-E. (2016). The Differential DRP1 Phosphorylation and Mitochondrial Dynamics in the Regional Specific Astroglial Death Induced by Status Epilepticus. Front. Cell. Neurosci.

Kornmann, B., Currie, E., Collins, S.R., Schuldiner, M., Nunnari, J., Weissman, J.S., and Walter, P. (2009). An ER-mitochondria tethering complex revealed by a synthetic biology screen. Science (80-. ). 325, 477-481.

Korobova, F., Ramabhadran, V., and Higgs, H.N. (2013). An actin-dependent step in mitochondrial fission mediated by the ER-associated formin INF2. Science (80-. ). 339, 464467.

Koshiba, T. (2004). Structural basis of mitochondrial tethering by mitofusin complexes. Science (80-. ). 305, 858-862.

Kraus, F., and Ryan, M.T. (2017). The constriction and scission machineries involved in mitochondrial fission. J. Cell Sci. 130, 2953-2960.

Krebs, H.A., and Johnson, W.A. (1937). The role of citric acid in intermediary metabolism in animal tissue. Enzymologia 4, 148-156.

Kumar, N., Leonzino, M., Hancock-Cerutti, W., Horenkamp, F.A., Li, P., Lees, J.A., Wheeler, H., Reinisch, K.M., and De Camilli, P. (2018). VPS13A and VPS13C are lipid transport proteins 
differentially localized at ER contact sites. J. Cell Biol. 217, 3625-3639.

Labrousse, A.M., Zappaterra, M.D., Rube, D.A., and van der Bliek, A.M. (1999). C. elegans dynamin-related protein DRP-1 controls severing of the mitochondrial outer membrane. Mol. Cell 4, 815-826.

Laderoute, K.R., Amin, K., Calaoagan, J.M., Knapp, M., Le, T., Orduna, J., Foretz, M., and Viollet, B. (2006). 5'-AMP-Activated Protein Kinase (AMPK) Is Induced by Low-Oxygen and Glucose Deprivation Conditions Found in Solid-Tumor Microenvironments. Mol. Cell. Biol.

Lahiri, S., Toulmay, A., and Prinz, W.A. (2015). Membrane contact sites, gateways for lipid homeostasis. Curr. Opin. Cell Biol. 33, 82-87.

Lang, A., John Peter, A.T., and Kornmann, B. (2015). ER-mitochondria contact sites in yeast: Beyond the myths of ERMES. Curr. Opin. Cell Biol. 35, 7-12.

Laplante, M., and Sabatini, D.M. (2009). An Emerging Role of mTOR in Lipid Biosynthesis. Curr. Biol. 19, R1046-R1052.

Lev, S. (2010). Non-vesicular lipid transport by lipid-transfer proteins and beyond. Nat. Rev. Mol. Cell Biol. 11, 739-750.

LEWIS, M.R., and LEWIS, W.H. (1914). Mitochondria in tissue culture. Science (80-. ). 39, 330333.

Lieberthal, W., Tang, M., Zhang, L., Viollet, B., Patel, V., and Levine, J.S. (2013). Susceptibility to ATP depletion of primary proximal tubular cell cultures derived from mice lacking either the $\alpha 1$ or the $\alpha 2$ isoform of the catalytic domain of AMPK. BMC Nephrol. 14, 251.

Liesa, M., Palacín, M., and Zorzano, A. (2009). Mitochondrial Dynamics in Mammalian Health and Disease. Physiol. Rev. 89, 799-845.

Lin, M.T., and Beal, M.F. (2006). Mitochondrial dysfunction and oxidative stress in neurodegenerative diseases. Nature 443, 787-795.

Liou, J., Kim, M.L., Do Heo, W., Jones, J.T., Myers, J.W., Ferrell, J.E., and Meyer, T. (2005). STIM Is a Ca2+ Sensor Essential for Ca2+-Store-Depletion-Triggered Ca2+ Influx. Curr. Biol. 15, 12351241.

Liou, J., Fivaz, M., Inoue, T., and Meyer, T. (2007). Live-cell imaging reveals sequential oligomerization and local plasma membrane targeting of stromal interaction molecule 1 after Ca2+ store depletion. Proc. Natl. Acad. Sci. 104, 9301-9306. 
Liu, R., and Chan, D.C. (2015). The mitochondrial fission receptor Mff selectively recruits oligomerized Drp1. Mol. Biol. Cell 26, 4466-4477.

Liu, X., Kim, C.N., Yang, J., Jemmerson, R., and Wang, X. (1996). Induction of apoptotic program in cell-free extracts: Requirement for dATP and cytochrome c. Cell 86, 147-157.

Lodhi, I.J., and Semenkovich, C.F. (2014). Peroxisomes: A nexus for lipid metabolism and cellular signaling. Cell Metab.

López-Crisosto, C., Bravo-Sagua, R., Rodriguez-Peña, M., Mera, C., Castro, P.F., Quest, A.F.G., Rothermel, B.A., Cifuentes, M., and Lavandero, S. (2015). ER-to-mitochondria miscommunication and metabolic diseases. Biochim. Biophys. Acta - Mol. Basis Dis. 1852, 2096-2105.

Losón, O.C., Song, Z., Chen, H., and Chan, D.C. (2013). Fis1, Mff, MiD49, and MiD51 mediate Drp1 recruitment in mitochondrial fission. Mol. Biol. Cell 24, 659-667.

Lou, P.-H., Hansen, B.S., Olsen, P.H., Tullin, S., Murphy, M.P., and Brand, M.D. (2007). Mitochondrial uncouplers with an extraordinary dynamic range. Biochem. J. 407, 129-140. Lynes, E.M., Raturi, A., Shenkman, M., Sandoval, C.O., Yap, M.C., Wu, J., Janowicz, A., Myhill, N., Benson, M.D., Campbell, R.E., et al. (2013). Palmitoylation is the switch that assigns calnexin to quality control or ER Ca2+ signaling. J. Cell Sci. 126, 3893-3903.

Manor, U., Bartholomew, S., Golani, G., Christenson, E., Kozlov, M., Higgs, H., Spudich, J., and Lippincott-Schwartz, J. (2015). A mitochondria-anchored isoform of the actin-nucleating spire protein regulates mitochondrial division. Elife 4.

Matsuzaki, H., Fujimoto, T., Tanaka, M., and Shirasawa, S. (2013). Tespa1 is a novel component of mitochondria-associated endoplasmic reticulum membranes and affects mitochondrial calcium flux. Biochem. Biophys. Res. Commun. 433, 322-326.

McGarry, J.D., Leatherman, G.F., and Foster, D.W. (1978). Carnitine palmitoyltransferase I. The site of inhibition of hepatic fatty acid oxidation by malonyl-CoA. J. Biol. Chem.

Van Meer, G., Voelker, D.R., and Feigenson, G.W. (2008). Membrane lipids: Where they are and how they behave. Nat. Rev. Mol. Cell Biol.

Meyer, D.I., Krause, E., and Dobberstein, B. (1982). Secretory protein translocation across membranes - The role of the "docking protein." Nature.

Mihaylova, M.M., and Shaw, R.J. (2011). The AMPK signalling pathway coordinates cell growth, autophagy and metabolism. Nat. Cell Biol. 
Mitchell, P., and Moyle, J. (1969). Estimation of Membrane Potential and pH Difference across the Cristae Membrane of Rat Liver Mitochondria. Eur. J. Biochem. 7, 471-484.

MITCHELL, P. (1961). Coupling of phosphorylation to electron and hydrogen transfer by a chemi-osmotic type of mechanism. Nature 191, 144-148.

Murley, A., Lackner, L.L., Osman, C., West, M., Voeltz, G.K., Walter, P., and Nunnari, J. (2013). ER-associated mitochondrial division links the distribution of mitochondria and mitochondrial DNA in yeast. Elife 2 .

Murphy, M.P. (2009). How mitochondria produce reactive oxygen species. Biochem. J. 417, 113.

Naon, D., and Scorrano, L. (2014). At the right distance: ER-mitochondria juxtaposition in cell life and death. Biochim. Biophys. Acta - Mol. Cell Res. 1843, 2184-2194.

Naon, D., Zaninello, M., Giacomello, M., Varanita, T., Grespi, F., Lakshminaranayan, S., Serafini, A., Semenzato, M., Herkenne, S., Hernández-Alvarez, M.I., et al. (2016). Critical reappraisal confirms that Mitofusin 2 is an endoplasmic reticulum-mitochondria tether. Proc. Natl. Acad. Sci. 113, 11249-11254.

Ngoh, G.A., Papanicolaou, K.N., and Walsh, K. (2012). Loss of mitofusin 2 promotes endoplasmic reticulum stress. J. Biol. Chem. 287, 20321-20332.

Nunnari, J., and Suomalainen, A. (2012). Mitochondria: In Sickness and in Health. Cell 148, 1145-1159.

O’Neill, H.M., Maarbjerg, S.J., Crane, J.D., Jeppesen, J., Jorgensen, S.B., Schertzer, J.D., Shyroka, O., Kiens, B., van Denderen, B.J., Tarnopolsky, M.A., et al. (2011). AMP-activated protein kinase (AMPK) 12 muscle null mice reveal an essential role for AMPK in maintaining mitochondrial content and glucose uptake during exercise. Proc. Natl. Acad. Sci. 108, 16092-16097.

Otera, H., Wang, C., Cleland, M.M., Setoguchi, K., Yokota, S., Youle, R.J., and Mihara, K. (2010). Mff is an essential factor for mitochondrial recruitment of Drp1 during mitochondrial fission in mammalian cells. J. Cell Biol. 191, 1141-1158.

Oude Weernink, P.A., Han, L., Jakobs, K.H., and Schmidt, M. (2007). Dynamic phospholipid signaling by G protein-coupled receptors. Biochim. Biophys. Acta - Biomembr. 1768, 888-900.

Paillusson, S., Stoica, R., Gomez-suaga, P., Lau, D.H.W., Mueller, S., Miller, T., and Miller, C.C.J. (2016). There's Something Wrong with my MAM ; the ER - Mitochondria Axis and Neurodegenerative Diseases. 39, 146-157. 
Palade, G.E. (1956). THE ENDOPLASMIC RETICULUM. J. Cell Biol. 2, 85-98.

Palmer, A.E., and Tsien, R.Y. (2006). Measuring calcium signaling using genetically targetable fluorescent indicators. Nat. Protoc. 1, 1057-1065.

Palmer, A.E., Giacomello, M., Kortemme, T., Hires, S.A., Lev-Ram, V., Baker, D., and Tsien, R.Y. (2006). Ca2+ Indicators Based on Computationally Redesigned Calmodulin-Peptide Pairs. Chem. Biol. 13, 521-530.

Palmer, C.S., Elgass, K.D., Parton, R.G., Osellame, L.D., Stojanovski, D., and Ryan, M.T. (2013). Adaptor Proteins MiD49 and MiD51 Can Act Independently of Mff and Fis1 in Drp1 Recruitment and Are Specific for Mitochondrial Fission. J. Biol. Chem. 288, 27584-27593. Park, S.H., and Blackstone, C. (2010). Further assembly required: construction and dynamics of the endoplasmic reticulum network. EMBO Rep. 11, 515-521.

Patergnani, S., Suski, J.M., Agnoletto, C., Bononi, A., Bonora, M., De Marchi, E., Giorgi, C., Marchi, S., Missiroli, S., Poletti, F., et al. (2011). Calcium signaling around Mitochondria Associated Membranes (MAMs). Cell Commun. Signal. 9, 19.

Peeters, A., Shinde, A.B., Dirkx, R., Smet, J., De Bock, K., Espeel, M., Vanhorebeek, I., Vanlander, A., Van Coster, R., Carmeliet, P., et al. (2015). Mitochondria in peroxisome-deficient hepatocytes exhibit impaired respiration, depleted DNA, and PGC-1 $\alpha$ independent proliferation. Biochim. Biophys. Acta - Mol. Cell Res. 1853, 285-298.

Pellegrino, M.W., Nargund, A.M., and Haynes, C.M. (2013). Signaling the mitochondrial unfolded protein response. Biochim. Biophys. Acta - Mol. Cell Res. 1833, 410-416.

Peretti, D., Dahan, N., Shimoni, E., Hirschberg, K., and Lev, S. (2008). Coordinated Lipid Transfer between the Endoplasmic Reticulum and the Golgi Complex Requires the VAP Proteins and Is Essential for Golgi-mediated Transport. Mol. Biol. Cell 19, 3871-3884.

Pernas, L., and Scorrano, L. (2016). Mito-Morphosis: Mitochondrial Fusion, Fission, and Cristae Remodeling as Key Mediators of Cellular Function. Annu. Rev. Physiol. 78, 505-531.

Petrungaro, C., and Kornmann, B. (2019). Lipid exchange at ER-mitochondria contact sites: a puzzle falling into place with quite a few pieces missing. Curr. Opin. Cell Biol. 57, 71-76.

Pinaud, F., and Dahan, M. (2011). Targeting and imaging single biomolecules in living cells by complementation-activated light microscopy with split-fluorescent proteins. Proc. Natl. Acad. Sci. 108, E201-E210.

Pinton, P., Giorgi, C., Siviero, R., Zecchini, E., and Rizzuto, R. (2008). Calcium and apoptosis: ER- 
mitochondria Ca2+ transfer in the control of apoptosis. Oncogene 27, 6407-6418.

Plaut, G., and S. Sung (1954). Diphosphopyridine nucleotide isocitric dehydrogenase from animal tissues. J. Biol. Chem. 207, 305-314.

Plotegher, N., and Duchen, M.R. (2017). Mitochondrial Dysfunction and Neurodegeneration in Lysosomal Storage Disorders. Trends Mol. Med. 23, 116-134.

Qi, Y., Yan, L., Yu, C., Guo, X., Zhou, X., Hu, X., Huang, X., Rao, Z., Lou, Z., and Hu, J. (2016). Structures of human mitofusin 1 provide insight into mitochondrial tethering. J. Cell Biol. 215, 621-629.

Raben, N., Wong, A., Ralston, E., and Myerowitz, R. (2012). Autophagy and mitochondria in Pompe disease: Nothing is so new as what has long been forgotten. Am. J. Med. Genet. Part C Semin. Med. Genet. 160C, 13-21.

Rabinovitch, R.C., Samborska, B., Faubert, B., Pause, A., St, J., Jones, R.G., Rabinovitch, R.C., Samborska, B., Faubert, B., Ma, E.H., et al. (2017). Report AMPK Maintains Cellular Metabolic Homeostasis through Regulation of Mitochondrial Reactive Report AMPK Maintains Cellular Metabolic Homeostasis through Regulation of Mitochondrial Reactive Oxygen Species. 1-9. Raimundo, N. (2014). Mitochondrial pathology: Stress signals from the energy factory. Trends Mol. Med. 20, 282-292.

Raimundo, N., Baysal, B.E., and Shadel, G.S. (2011). Revisiting the TCA cycle: signaling to tumor formation. Trends Mol. Med. 17, 641-649.

Raimundo, N., Song, L., Shutt, T.E., McKay, S.E., Cotney, J., Guan, M.-X., Gilliland, T.C., Hohuan, D., Santos-Sacchi, J., and Shadel, G.S. (2012). Mitochondrial Stress Engages E2F1 Apoptotic Signaling to Cause Deafness. Cell 148, 716-726.

Rambold, A.S., Kostelecky, B., Elia, N., and Lippincott-Schwartz, J. (2011). Tubular network formation protects mitochondria from autophagosomal degradation during nutrient starvation. Proc. Natl. Acad. Sci. 108, 10190-10195.

Rapizzi, E., Pinton, P., Szabadkai, G., Wieckowski, M.R., Vandecasteele, G., Baird, G., Tuft, R.A., Fogarty, K.E., and Rizzuto, R. (2002). Recombinant expression of the voltage-dependent anion channel enhances the transfer of Ca 2+ microdomains to mitochondria. J. Cell Biol. 159, 613624.

Rasola, A., and Bernardi, P. (2011). Mitochondrial permeability transition in Ca2+-dependent apoptosis and necrosis. Cell Calcium 50, 222-233. 
Raturi, A., and Simmen, T. (2013). Where the endoplasmic reticulum and the mitochondrion tie the knot: The mitochondria-associated membrane (MAM). Biochim. Biophys. Acta - Mol. Cell Res. 1833, 213-224.

Rizzuto, R. (1998). Close Contacts with the Endoplasmic Reticulum as Determinants of Mitochondrial Ca2+ Responses. Science (80-. ). 280, 1763-1766.

Rizzuto, R., Simpson, A.W.M., Brini, M., and Pozzan, T. (1992). Rapid changes of mitochondrial Ca2+ revealed by specifically targeted recombinant aequorin. Nature 358, 325-327.

Rizzuto, R., Marchi, S., Bonora, M., Aguiari, P., Bononi, A., De Stefani, D., Giorgi, C., Leo, S., Rimessi, A., Siviero, R., et al. (2009). Ca2+ transfer from the ER to mitochondria: When, how and why. Biochim. Biophys. Acta - Bioenerg. 1787, 1342-1351.

Rizzuto, R., De Stefani, D., Raffaello, A., and Mammucari, C. (2012). Mitochondria as sensors and regulators of calcium signalling. Nat. Rev. Mol. Cell Biol. 13, 566-578.

Rochin, L., Sauvanet, C., Jääskeläinen, E., Houcine, A., Kivelä, A., Ma, X., Marien, E., Dehairs, J., Neveu, J., Bars, R. Le, et al. (2019). ORP5 Regulates Transport of Lipids and Calcium to Mitochondria at Endoplasmic Reticulum-Mitochondria Membrane Contact Sites. BioRxiv. Rossi, A., Pizzo, P., and Filadi, R. (2019). Calcium, mitochondria and cell metabolism: A functional triangle in bioenergetics. Biochim. Biophys. Acta - Mol. Cell Res. 1866, 1068-1078. Ruggiano, A., Foresti, O., and Carvalho, P. (2014). ER-associated degradation: Protein quality control and beyond. J. Cell Biol. 204, 869-879.

Sagan, L. (1967). On the origin of mitosing cells. J. Theor. Biol. 14, 225-IN6.

Saggerson, D. (2008). Malonyl-CoA, a Key Signaling Molecule in Mammalian Cells. Annu. Rev. Nutr. 28, 253-272.

Salinas, S., Proukakis, C., Crosby, A., and Warner, T.T. (2008). Hereditary spastic paraplegia: clinical features and pathogenetic mechanisms. Lancet Neurol. 7, 1127-1138.

Sassano, M.L., van Vliet, A.R., and Agostinis, P. (2017). Mitochondria-Associated Membranes As Networking Platforms and Regulators of Cancer Cell Fate. Front. Oncol. 7, 1-16.

Scalettar, B.A., Abney, J.R., and Hackenbrock, C.R. (1991). Dynamics, structure, and function are coupled in the mitochondrial matrix. Proc. Natl. Acad. Sci. 88, 8057-8061.

Scheffler, I.E. (2008). Mitochondria (Hoboken, New Jersey: John Wiley \& Sons, Inc.).

Schreiner, B., Hedskog, L., Wiehager, B., and Ankarcrona, M. (2014). Amyloid- $\beta$ peptides are 
generated in mitochondria-associated endoplasmic reticulum membranes. J. Alzheimer's Dis. 43, 369-374.

Schulz, T.A., and Prinz, W.A. (2007). Sterol transport in yeast and the oxysterol binding protein homologue (OSH) family. Biochim. Biophys. Acta - Mol. Cell Biol. Lipids 1771, 769-780.

Schwarz, D.S., and Blower, M.D. (2016). The endoplasmic reticulum: structure, function and response to cellular signaling. Cell. Mol. Life Sci. 73, 79-94.

Scorrano, L., De Matteis, M.A., Emr, S., Giordano, F., Hajnóczky, G., Kornmann, B., Lackner, L.L., Levine, T.P., Pellegrini, L., Reinisch, K., et al. (2019). Coming together to define membrane contact sites. Nat. Commun. 10, 1287.

Selak, M.A., de Chadarevian, J.P., Melvin, J.J., Grover, W.D., Salganicoff, L., and Kaye, E.M. (2000). Mitochondrial activity in Pompe's disease. Pediatr. Neurol. 23, 54-57.

Sena, L.A., and Chandel, N.S. (2012). Physiological roles of mitochondrial reactive oxygen species. Mol. Cell 48, 158-167.

Shen, Q., Yamano, K., Head, B.P., Kawajiri, S., Cheung, J.T.M., Wang, C., Cho, J.-H., Hattori, N., Youle, R.J., and van der Bliek, A.M. (2014). Mutations in Fis1 disrupt orderly disposal of defective mitochondria. Mol. Biol. Cell 25, 145-159.

Shibata, Y., Voss, C., Rist, J.M., Hu, J., Rapoport, T.A., Prinz, W.A., and Voeltz, G.K. (2008). The Reticulon and Dp1/Yop1p Proteins Form Immobile Oligomers in the Tubular Endoplasmic Reticulum. J. Biol. Chem. 283, 18892-18904.

Shibata, Y., Shemesh, T., Prinz, W.A., Palazzo, A.F., Kozlov, M.M., and Rapoport, T.A. (2010). Mechanisms Determining the Morphology of the Peripheral ER. Cell 143, 774-788.

Shibutani, S.T., and Yoshimori, T. (2014). A current perspective of autophagosome biogenesis. Cell Res. 24, 58-68.

Shioda, N., Ishikawa, K., Tagashira, H., Ishizuka, T., Yawo, H., and Fukunaga, K. (2012). Expression of a truncated form of the endoplasmic reticulum chaperone protein, $\sigma 1$ receptor, promotes mitochondrial energy depletion and apoptosis. J. Biol. Chem. 287, 23318-23331. Simmen, T., Aslan, J.E., Blagoveshchenskaya, A.D., Thomas, L., Wan, L., Xiang, Y., Feliciangeli, S.F., Hung, C.-H., Crump, C.M., and Thomas, G. (2005). PACS-2 controls endoplasmic reticulummitochondria communication and Bid-mediated apoptosis. EMBO J. 24, 717-729.

Singh, R., and Cuervo, A.M. (2011). Autophagy in the Cellular Energetic Balance. Cell Metab. $13,495-504$. 
Smirnova, E., Griparic, L., Shurland, D.-L., and van der Bliek, A.M. (2001). Dynamin-related Protein Drp1 Is Required for Mitochondrial Division in Mammalian Cells. Mol. Biol. Cell 12, 2245-2256.

Stefan, C.J., Manford, A.G., Baird, D., Yamada-Hanff, J., Mao, Y., and Emr, S.D. (2011). Osh proteins regulate phosphoinositide metabolism at ER-plasma membrane contact sites. Cell $144,389-401$.

De Stefani, D., Raffaello, A., Teardo, E., Szabò, I., and Rizzuto, R. (2011). A forty-kilodalton protein of the inner membrane is the mitochondrial calcium uniporter. Nature 476, 336-340. Stern, J.R., and Ochoa, S. (1949). Enzymatic synthesis of citric acid by condensation of acetate and oxalacetate. J. Biol. Chem. 179, 491.

Stern, J.R., Ochoa, S., and Lynen, F. (1952). Enzymatic synthesis of citric acid. V. Reaction of acetyl coenzyme A. J. Biol. Chem. 198, 313-321.

Stoica, R., De Vos, K.J., Paillusson, S., Mueller, S., Sancho, R.M., Lau, K.-F., Vizcay-Barrena, G., Lin, W.-L., Xu, Y.-F., Lewis, J., et al. (2014). ER-mitochondria associations are regulated by the VAPB-PTPIP51 interaction and are disrupted by ALS/FTD-associated TDP-43. Nat. Commun. 5, 3996.

Stone, S.J., and Vance, J.E. (2000). Phosphatidylserine synthase- 1 and -2 are localized to mitochondria-associated membranes. J. Biol. Chem. 275, 34534-34540.

Szabadkai, G., Bianchi, K., Várnai, P., De Stefani, D., Wieckowski, M.R., Cavagna, D., Nagy, A.I., Balla, T., and Rizzuto, R. (2006). Chaperone-mediated coupling of endoplasmic reticulum and mitochondrial Ca 2+ channels. J. Cell Biol. 175, 901-911.

Taguchi, N., Ishihara, N., Jofuku, A., Oka, T., and Mihara, K. (2007). Mitotic phosphorylation of dynamin-related GTPase Drp1 participates in mitochondrial fission. J. Biol. Chem. 282, 1152111529.

Tamura, Y., and Endo, T. (2017). Role of intra- and inter-mitochondrial membrane contact sites in yeast phospholipid biogenesis. In Advances in Experimental Medicine and Biology, pp. 121133.

Tatsuta, T., Scharwey, M., and Langer, T. (2014). Mitochondrial lipid trafficking. Trends Cell Biol. 24, 44-52.

Terada, H. (1990). Uncouplers of oxidative phosphorylation. In Environmental Health Perspectives, $\mathrm{p}$. 
Terasaki, M. (1986). Microtubules and the endoplasmic reticulum are highly interdependent structures. J. Cell Biol. 103, 1557-1568.

Territo, P.R., Mootha, V.K., French, S.A., and Balaban, R.S. (2017). Ca 2+ activation of heart mitochondrial oxidative phosphorylation: role of the F 0 /F 1 -ATPase . Am. J. Physiol. Physiol. Theurey, P., and Rieusset, J. (2017). Mitochondria-Associated Membranes Response to Nutrient Availability and Role in Metabolic Diseases. Trends Endocrinol. Metab. 28, 32-45. Tilokani, L., Nagashima, S., Paupe, V., and Prudent, J. (2018). Mitochondrial dynamics: overview of molecular mechanisms. Essays Biochem. 62, 341-360.

Tooze, S.A., and Yoshimori, T. (2010). The origin of the autophagosomal membrane. Nat. Cell Biol. 12, 831-835.

Toyama, E.Q., Herzig, S., Courchet, J., Jr, T.L.L., Oliver, C., Hellberg, K., Young, N.P., Chen, H., Polleux, F., David, C., et al. (2016). AMP-activated protein kinase mediates mitochondrial fission in response to energy stress. 351, 275-281.

Tubbs, E., Theurey, P., Vial, G., Bendridi, N., Bravard, A., Chauvin, M.-A., Ji-Cao, J., Zoulim, F., Bartosch, B., Ovize, M., et al. (2014). Mitochondria-associated endoplasmic reticulum membrane (MAM) integrity is required for insulin signaling and is implicated in hepatic insulin resistance. Diabetes 63, 3279-3294.

Valko, M., Leibfritz, D., Moncol, J., Cronin, M.T.D., Mazur, M., and Telser, J. (2007). Free radicals and antioxidants in normal physiological functions and human disease. Int. J. Biochem. Cell Biol. 39, 44-84.

Vance, J.E. (1990). Phospholipid synthesis in a membrane fraction associated with mitochondria. J. Biol. Chem.

Vance, J.E., and Tasseva, G. (2013). Formation and function of phosphatidylserine and phosphatidylethanolamine in mammalian cells. Biochim. Biophys. Acta - Mol. Cell Biol. Lipids $1831,543-554$.

van Vliet, A.R., Verfaillie, T., and Agostinis, P. (2014). New functions of mitochondria associated membranes in cellular signaling. Biochim. Biophys. Acta - Mol. Cell Res. 1843, 2253-2262.

Voeltz, G.K., Prinz, W.A., Shibata, Y., Rist, J.M., and Rapoport, T.A. (2006). A Class of Membrane Proteins Shaping the Tubular Endoplasmic Reticulum. Cell 124, 573-586.

De Vos, K.J., Mórotz, G.M., Stoica, R., Tudor, E.L., Lau, K.-F., Ackerley, S., Warley, A., Shaw, C.E., and Miller, C.C.J. (2012). VAPB interacts with the mitochondrial protein PTPIP51 to regulate 
calcium homeostasis. Hum. Mol. Genet. 21, 1299-1311.

Wai, T., and Langer, T. (2016). Mitochondrial Dynamics and Metabolic Regulation. Trends Endocrinol. Metab. 27, 105-117.

Wakabayashi, J., Zhang, Z., Wakabayashi, N., Tamura, Y., Fukaya, M., Kensler, T.W., lijima, M., and Sesaki, H. (2009). The dynamin-related GTPase Drp1 is required for embryonic and brain development in mice. J. Cell Biol. 186, 805-816.

Walter, P. (1981a). Translocation of proteins across the endoplasmic reticulum. I. Signal recognition protein (SRP) binds to in-vitro-assembled polysomes synthesizing secretory protein. J. Cell Biol. 91, 545-550.

Walter, P. (1981b). Translocation of proteins across the endoplasmic reticulum. II. Signal recognition protein (SRP) mediates the selective binding to microsomal membranes to in-vitroassembled polysomes synthesizing secretory protein. J. Cell Biol. 91, 551-556.

Wang, X., Wang, W., Li, L., Perry, G., Lee, H., and Zhu, X. (2014). Oxidative stress and mitochondrial dysfunction in Alzheimer's disease. Biochim. Biophys. Acta - Mol. Basis Dis. $1842,1240-1247$.

Wieckowski, M.R., Giorgi, C., Lebiedzinska, M., Duszynski, J., and Pinton, P. (2009). Isolation of mitochondria-associated membranes and mitochondria from animal tissues and cells. Nat. Protoc. 4, 1582-1590.

Wong, Y.C., Ysselstein, D., and Krainc, D. (2018). Mitochondria-lysosome contacts regulate mitochondrial fission via RAB7 GTP hydrolysis. Nature 554, 382-386.

Wozniak, M.J., Bola, B., Brownhill, K., Yang, Y.-C., Levakova, V., and Allan, V.J. (2009). Role of kinesin-1 and cytoplasmic dynein in endoplasmic reticulum movement in VERO cells. J. Cell Sci. $122,1979-1989$.

Wu, H., Carvalho, P., and Voeltz, G.K. (2018). Here, there, and everywhere: The importance of ER membrane contact sites. Science (80-. ). 361, eaan5835.

Yambire, K.F., Fernandez-Mosquera, L., Steinfeld, R., Mühle, C., Ikonen, E., Milosevic, I., and Raimundo, N. (2019). Mitochondrial biogenesis is transcriptionally repressed in lysosomal lipid storage diseases. Elife 8.

Yan, M., Gingras, M.-C., Dunlop, E.A., Nouët, Y., Dupuy, F., Jalali, Z., Possik, E., Coull, B.J., Kharitidi, D., Dydensborg, A.B., et al. (2014). The tumor suppressor folliculin regulates AMPKdependent metabolic transformation. J. Clin. Invest. 124, 2640-2650. 
Yang, J.-Y., and Yang, W.Y. (2013). Bit-by-bit autophagic removal of parkin-labelled mitochondria. Nat. Commun. 4, 2428.

Yoon, Y., Pitts, K.R., and McNiven, M.A. (2001). Mammalian dynamin-like protein DLP1 tubulates membranes. Mol. Biol. Cell 12, 2894-2905.

Yu, W., Sun, Y., Guo, S., and Lu, B. (2011). The PINK1/Parkin pathway regulates mitochondrial dynamics and function in mammalian hippocampal and dopaminergic neurons. Hum. Mol. Genet. 20, 3227-3240.

Zalman, L.S., Nikaido, H., and Kagawa, Y. (1980). Mitochondrial outer membrane contains a protein producing nonspecific diffusion channels. J. Biol. Chem. 255, 1771-1774.

Zampese, E., Fasolato, C., Kipanyula, M.J., Bortolozzi, M., Pozzan, T., and Pizzo, P. (2011). Presenilin 2 modulates endoplasmic reticulum (ER)-mitochondria interactions and Ca2+ crosstalk. Proc. Natl. Acad. Sci. 108, 2777-2782.

van der Zand, A., Gent, J., Braakman, I., and Tabak, H.F. (2012). Biochemically Distinct Vesicles from the Endoplasmic Reticulum Fuse to Form Peroxisomes. Cell 149, 397-409.

Zhao, X., Alvarado, D., Rainier, S., Lemons, R., Hedera, P., Weber, C.H., Tukel, T., Apak, M., Heiman-Patterson, T., Ming, L., et al. (2001). Mutations in a newly identified GTPase gene cause autosomal dominant hereditary spastic paraplegia. Nat. Genet. 29, 326-331. 